\title{
Involuções e o teorema de Borsuk-Ulam para algumas variedades de dimensão 4
}

Anderson Paião dos Santos

TESE APRESEnTADA

$\mathrm{AO}$

Instituto De Matemática e EstatísticA

DA

Universidade de SÃo Paulo

PARA

OBTENÇÃO DO TÍTULO

$\mathrm{DE}$

Doutor EM CiÊnCIAS

Programa: Matemática

Orientador: Prof. Dr. Daciberg Lima Gonçalves

Durante o desenvolvimento deste trabalho o autor recebeu auxílio financeiro do CNPq

São Paulo, fevereiro de 2012 


\title{
Involuções e o teorema de Borsuk-Ulam para algumas variedades de dimensão 4
}

\author{
Esta tese contém as correções e alterações \\ sugeridas pela Comissão Julgadora durante a defesa \\ realizada por Anderson Paião dos Santos em 29/02/2012. \\ O original encontra-se disponível no Instituto de \\ Matemática e Estatística da Universidade de São Paulo.
}

Comissão Julgadora:

- Prof. Dr. Daciberg Lima Gonçalves (orientador) - IME-USP

- Profa. Dra. Lucília Daruiz Borsari - IME-USP

- Profa. Dra. Ermínia de Lourdes Campello Fanti - IBILCE

- Prof. Dr. Pedro Luiz Queiroz Pergher - UFSCar

- Prof. Dr. João Peres Vieira - UNESP 


\section{Agradecimentos}

Agradeço a Deus, por me conceder a graça de concluir mais esta jornada, por ter me fortalecido nos momentos que mais precisei e pelas pessoas que estiveram comigo nesta caminhada e que com certeza foram fundamentais para esta conquista. Em especial quero deixar aqui meus sinceros agradecimentos:

À minha mãe Albertina, pela educação que me deu, pelo apoio em todas as minhas decisões e pelas orações.

Ao Prof. Dr. Daciberg Lima Gonçalves, por ter aceito me orientar, pelas disponibilidade e paciência que teve em todos os momentos que precisei de sua ajuda, e pelos conhecimentos transmitidos.

À Prof ${ }^{-\mathrm{a}}$ Dr $\stackrel{\mathrm{a}}{-}$ Lucília Daruiz Borsari, pelo apoio, pelos conselhos e disponibilidade nos momentos que precisei de sua ajuda. Aos professores do IME, em particular às Prof $\frac{\text { as }}{}$ Dr $\underline{\text { as }}$ Rosa M. S. B. Chaves e Fernanda Cardona. Ao Prof. Dr. Edivaldo Lopes dos Santos pelas sugestões durante o exame de qualificação. Aos membros da banca pelas sugestões da redação final desta tese.

Aos amigos do IME, em particular aos amigos Oscar e Gustavo pelo apoio e valiosas

conversas. Às amigas Michele e Fernanda pelo agradável convívio. À minha irmã Nyejda, pelo apoio e torcida.

Ao $\mathrm{CNPq}$ pelo apoio financeiro.

Que Deus os abençoe. 


\section{Resumo}

Na maior parte deste trabalho, estudamos a existência de involuções livres sobre algumas 4-variedades fechadas, com o mesmo tipo de homotopia do espaço total de algum fibrado de superfície sobre superfície, bem como uma generalização do teorema de Borsuk-Ulam para tais 4-variedades. Também estudamos a relação do teorema de Borsuk-Ulam, para aplicações da $n$-esfera em variedades, com a teoria de conível.

Palavras-chave: involução livre, Borsuk-Ulam, fibrado de superfície, conível. 


\section{Abstract}

In the most part of this work, we study the existence of free involutions over some closed 4-manifolds with the same homotopy type of the total space of some surface bundle over surface, as well as a generalization of the Borsuk-Ulam theorem for such 4-manifolds. Also we study the relation of the Borsuk-Ulam theorem, for maps from the $n$-sphere into manifolds, with the colevel theory.

Keywords: free involution, Borsuk-Ulam, surface bundle, colevel. 


\section{Sumário}

Lista de símbolos vi

Introdução viii

\begin{tabular}{lll}
\hline & Preliminares & 1
\end{tabular}

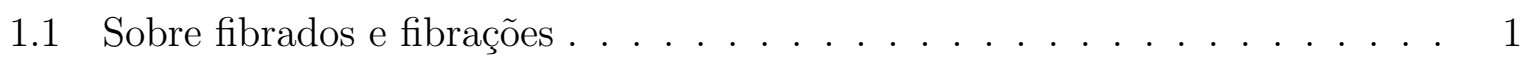

1.2 Espaços com involuções . . . . . . . . . . . . . . . . . . . . 3

$1.3 \quad$ A propriedade de Borsuk-Ulam (PBU) . . . . . . . . . . . . . . . . . 4

1.4 Nível e conível . . . . . . . . . . . . . . . . . . . . . . . . . . . . . . . 6

$\begin{array}{lll}2 & \text { PBU para produtos de superfícies } & 8\end{array}$

2.1 Introdução . . . . . . . . . . . . . . . . . . . . . . . . . . . 8

2.2 Involução diagonal $\ldots \ldots \ldots \ldots \ldots \ldots$

3 Fibrados de superfície sobre superfície e involuções livres 21

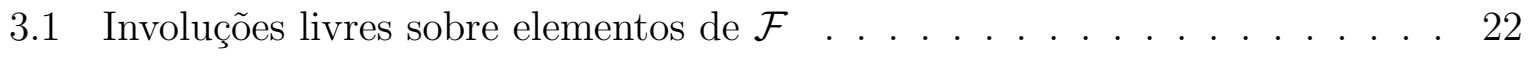

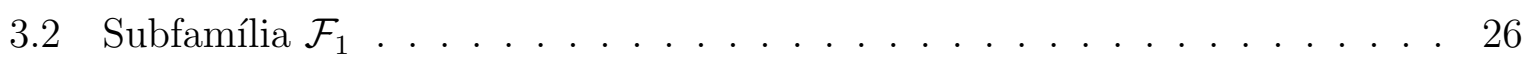

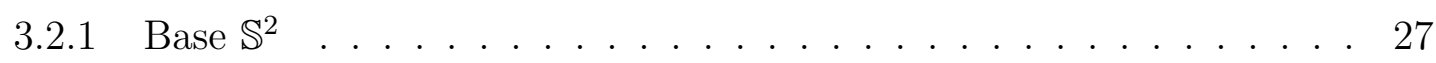

3.2 .2 Base $\mathbb{R} P^{2} \ldots \ldots \ldots \ldots \ldots \ldots$

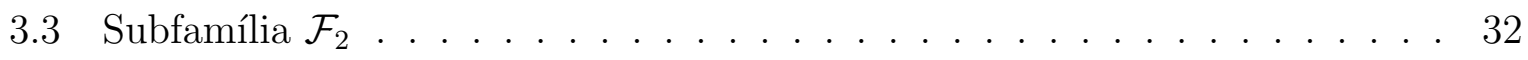

$3.3 .1 \quad F=\mathbb{S}^{2}$ e $B$ é $K(\pi, 1) \ldots \ldots \ldots \ldots$

$3.3 .2 \quad F=\mathbb{R} P^{2}$ e $B$ é $K(\pi, 1)$. . . . . . . . . . . . . . . . . 40

$3.3 .3 \quad F$ é $K(\pi, 1)$ e $B=\mathbb{S}^{2} \ldots \ldots \ldots \ldots \ldots$. . . . . . . . . . . . 45

$3.3 .4 \quad F$ é $K(\pi, 1)$ e $B=\mathbb{R} P^{2} \ldots \ldots \ldots \ldots \ldots$

3.4 Considerações sobre a subfamília $\mathcal{F}_{3} \ldots \ldots \ldots \ldots$ 
$4 \quad$ PBU para revestimentos duplos de fibrados de superfície 48

4.1 Resultados gerais . . . . . . . . . . . . . . . . . . . . . . . . . 48

$4.2 \quad$ PBU para revestimentos duplos de elementos de $\mathcal{F}_{1} \ldots \ldots \ldots$. . . . . . 50

$4.3 \quad$ PBU para revestimentos duplos de elementos de $\mathcal{F}_{2} \ldots \ldots \ldots$. . . . . . . . . . 51

$4.3 .1 \quad F=\mathbb{S}^{2}$ e $B$ é $K(\pi, 1) \ldots \ldots \ldots \ldots \ldots$. . . . . . . . . . . . 51

$4.3 .2 \quad F=\mathbb{R} P^{2}$ e $B$ é $K(\pi, 1) \ldots \ldots \ldots \ldots \ldots$. . . . . . . . . . . 52

$4.3 .3 \quad F$ é $K(\pi, 1)$ e $B=\mathbb{S}^{2} \ldots \ldots \ldots \ldots \ldots \ldots$

$4.3 .4 \quad F$ é $K(\pi, 1)$ e $B=\mathbb{R} P^{2} \ldots \ldots \ldots \ldots \ldots$. . . . . . . . . . . . . . . .

4.4 Considerações sobre a subfamília $\mathcal{F}_{3} \ldots \ldots \ldots \ldots$

$\begin{array}{lll}5 & \text { PBU para }\left(T^{4}, \tau ; \mathbb{R}^{n}\right) & 59\end{array}$

5.1 4-variedades "flat" orientáveis . . . . . . . . . . . . . . . . . . . . . 59

5.2 4-variedades "flat" não orientáveis . . . . . . . . . . . . . . . . 63

$5.3 \quad$ PBU para $\left(T^{4}, \tau ; \mathbb{R}^{n}\right) \ldots \ldots \ldots \ldots \ldots \ldots \ldots$

$6 \quad$ PBU para revestimentos duplos de $\frac{K b \times K b}{\tau \times \beta} \quad 69$

$6.1 \quad$ Revestimentos duplos de $\frac{K b \times K b}{\tau \times \beta}$. . . . . . . . . . . . . . . . . . . 69

$\begin{array}{lll}7 & \text { PBU para aplicações em variedades } & 76\end{array}$

7.1 Equivalência . . . . . . . . . . . . . . . . . . 76

$7.2 \quad$ PBU para $\left(\mathbb{S}^{n}, A ; M^{k}\right)$ e o conível de $\left(M^{k}\right)^{*} \ldots \ldots \ldots \ldots$. . . . . . . 77

\begin{tabular}{|lr}
\hline A Involuções livres sobre $K b \times K b$ & 80
\end{tabular}

A.1 Cálculos . . . . . . . . . . . . . . . . . . . . . . . . . . . . . . . 80

\begin{tabular}{lr}
\hline Referências Bibliográficas & 84
\end{tabular}

\begin{tabular}{lr}
\hline Índice Remissivo & 88
\end{tabular} 


\section{Lista de símbolos}

\begin{tabular}{|c|c|}
\hline$\cong$ & relação de isomorfismo \\
\hline$[a, b]$ & palavra $a b a^{-1} b^{-1}$ \\
\hline$[a, b]^{\prime}$ & palavra $a b a b^{-1}$ \\
\hline$N \rtimes_{\theta} H$ & $\begin{array}{l}\text { produto semidireto de um grupo } N \text { por um grupo } H \text {, } \\
\text { sob uma ação } \theta\end{array}$ \\
\hline$Z(G)$ & centro de um grupo $G$ \\
\hline$G_{a b}$ & abelianizado de um grupo $G$ \\
\hline $\mathbb{S}^{n}$ & esfera unitária de dimensão $n$ \\
\hline $\mathbb{M}$ & faixa de Möbius \\
\hline $\mathbb{R} P^{2}$ & plano projetivo real \\
\hline$K b$ & garrafa de Klein \\
\hline$T^{n}$ & toro $n$-dimensional \\
\hline$S_{g}$ & superfície orientável de gênus $g$ \\
\hline$N_{h}$ & superfície não orientável de gênus $h$ \\
\hline$I$ & intervalo fechado $[0,1]$ \\
\hline $\mathbb{D}^{2}$ & disco bidimensional \\
\hline$\smile$ & produto cup \\
\hline$\frown$ & produto cap \\
\hline$\approx$ & relação de homeomorfismo \\
\hline$\simeq$ & relação de mesmo tipo de homotopia \\
\hline$\chi(X)$ & característica de Euler de um espaço $X$ \\
\hline$\partial X$ & bordo de um espaço $X$ \\
\hline $\operatorname{int}(X)$ & interior de um espaço $X$ \\
\hline$\widehat{X}$ & espaço de revestimento duplo de um espaço $X$ \\
\hline$f_{\#}: \pi_{1}(X) \rightarrow \pi_{1}(Y)$ & homomorfismo induzido pela aplicação $f: X \rightarrow Y$ \\
\hline
\end{tabular}


$\amalg$

$\operatorname{dim}(X)$ união disjunta

dimensão de um espaço $X$ 


\section{Introdução}

Conjecturado por S. Ulam e provado por K. Borsuk, em [3], o teorema conhecido como teorema de Borsuk-Ulam estabelece que, para qualquer aplicação contínua $f: \mathbb{S}^{n} \rightarrow \mathbb{R}^{n}$, existe $x \in \mathbb{S}^{n}$ tal que ele e seu antípoda $-x$ são colapsados num mesmo ponto pela aplicação $f$, isto é, $f(x)=f(-x)$. Uma interpretação deste problema, para o caso particular $n=2$, diz que em qualquer instante sempre existem dois lugares na terra (antípodas) com mesmas temperatura e pressão. Existem diversas versões, muitas provas diferentes e várias generalizações que dão destaque a este teorema. Uma referência interessante que faz uma abordagem do assunto é [25].

Uma generalização natural para este teorema é trocar a esfera $\mathbb{S}^{n}$ por um espaço topológico $X$ equipado com uma involução livre $\tau$, e o espaço euclidiano $\mathbb{R}^{n}$ por um espaço $Y$, e assim, dada uma aplicação contínua $f: X \rightarrow Y$, procurar saber da existência de algum ponto $x \in X$ tal que $f(x)=f(\tau(x))$. Quando este fato acontece para toda aplicação contínua $f: X \rightarrow Y$, dizemos que a tripla $(X, \tau ; Y)$ satisfaz a propriedade de Borsuk-Ulam (PBU). Vários trabalhos têm sido realizados nesta direção, dentre os quais destacamos: [1], [11], [12], [13] e [14].

Neste trabalho, estudamos involuções livres sobre algumas 4-variedades fechadas que têm o mesmo tipo de homotopia do espaço total de algum fibrado de superfície sobre superfície, bem como a propriedade de Borsuk-Ulam para tais 4-variedades. Também estudamos a propriedade de Borsuk-Ulam, para aplicações da $n$-esfera em variedades, relacionando-a com a teoria de conível. Nos próximos parágrafos, fazemos um apanhado do que é feito nos capítulos e no apêndice deste trabalho.

O Capítulo 1 tem um caráter preliminar e visa fixar notações e apresentar ferramentas úteis para nosso estudo.

Em [11], Gonçalves classifica as involuções livres sobre uma superfície fechada $S$ e estuda a propriedade de Borsuk-Ulam para triplas do tipo $\left(S, \tau ; \mathbb{R}^{2}\right)$. Motivados por este trabalho, no Capítulo 2, estudamos a propriedade de Borsuk-Ulam para $\left(M_{1} \times M_{2}, \tau \times\right.$ 
$\beta ; \mathbb{R}^{n}$ ), onde $M_{1}$ e $M_{2}$ são superfícies fechadas e $\tau \times \beta$ é uma involução livre sobre $M_{1} \times$ $M_{2}$, dada por $(\tau \times \beta)(x, y)=(\tau(x), \beta(y))$ tal que $\tau$ e $\beta$ são involuções sobre $M_{1}$ e $M_{2}$, respectivamente, e pelo menos uma delas é livre. Chamamos uma tal $\tau \times \beta$ de involução diagonal. Basicamente, trabalhamos com as involuções diagonais $\tau_{1}:=\tau \times \beta$ tal que $\tau$ e $\beta$ são ambas livres, e $\tau_{2}:=\tau \times \beta$ tal que apenas $\tau$ é livre. Neste capítulo, obtemos alguns resultados gerais para o produto de dois espaços, equipado com tais involuções, e em particular para o produto de superfícies, sendo que os casos a serem analisados são $n=2,3,4$ em triplas do tipo $\left(M_{1} \times M_{2}, \tau \times \beta ; \mathbb{R}^{n}\right)$. Por exemplo, com o auxílio de um método algébrico, no caso $n=2$, provamos que se $M_{1}$ e $M_{2}$ são superfícies fechadas, então a propriedade de Borsuk-Ulam vale para $\left(M_{1} \times M_{2}, \tau_{1} ; \mathbb{R}^{2}\right)$ se, e somente se, ela também vale para $\left(M_{1}, \tau ; \mathbb{R}^{2}\right)$ e $\left(M_{2}, \beta ; \mathbb{R}^{2}\right)$ - Teoremas 2.7 e 2.8 . Enquanto que, para $n=3,4$, obtemos que a propriedade de Borsuk-Ulam não vale para $\left(M_{1} \times M_{2}, \tau_{1} ; \mathbb{R}^{n}\right)$, nem para $\left(M_{1} \times M_{2}, \tau_{2} ; \mathbb{R}^{n}\right)$ - Proposição 2.9 . Em geral, não sabemos se existem outras involuções livres sobre os produtos de superfícies fechadas $M_{1} \times M_{2}$ que não sejam da forma diagonal. Desta forma, apresentamos, no Apêndice, cálculos realizados na tentativa de se encontrar tais involuções, para o caso particular em que $M_{1}=M_{2}=K b$ (garrafa de Klein).

Com o objetivo de obtermos outras 4-variedades equipadas com involuções livres, no Capítulo 3, consideramos 4-variedades fechadas $M$ com o mesmo tipo de homotopia do espaço total $E$ de algum fibrado de superfície sobre superfície, o qual denotamos por $\xi_{M}=(E, B, F, p)$. Na verdade, nosso estudo se baseia no caso em que $B$ e $F$ são superfícies fechadas. A família de todas estas 4-variedades fechadas é denotada por $\mathcal{F}$. Observemos que os produtos de superfícies, estudados no Capítulo 2, pertencem a esta família. Mais precisamente, o estudo do capítulo em questão é feito da seguinte maneira: dados $M \in \mathcal{F}$ e $[\varphi]$ uma classe não nula de $H^{1}\left(M ; \mathbb{Z}_{2}\right)$, olhamos para $\varphi$ como um epimorfismo de $\pi_{1}(M)$ em $\mathbb{Z}_{2}$. Da teoria de revestimento, consideramos o revestimento duplo de $M$ associado a $\varphi, M_{\varphi}$, isto é, $\pi_{1}\left(M_{\varphi}\right)=\operatorname{Ker}(\varphi)$, equipado com a involução livre $\tau_{\varphi}$ dada pela "deck transformation". Se chamamos tal procedimento de "operação" 1 sobre elementos da família $\mathcal{F}$, provamos que a família $\mathcal{F}$ é fechada com relação à "operação" 1 (Teorema 3.5), ou seja, se $M \in \mathcal{F}$, então $M_{\varphi} \in \mathcal{F}$. E mais, um fibrado que corresponde a $M_{\varphi}$ é dado por $\xi_{M_{\varphi}}=\left(E_{\varphi}, B^{\prime}, F^{\prime}, p^{\prime}\right)$, onde $\left(E_{\varphi}, q\right)$ é o revestimento duplo de $E$ associado a $\varphi$ e as superfícies fechadas $F^{\prime}$ e $B^{\prime}$ estão relacionadas com $F$ e $B$, dependendo da composta $p \circ q$ ter uma certa propriedade ou não. Neste ponto, dividimos a família $\mathcal{F}$ em três subfamílias disjuntas: $\mathcal{F}_{1}, \mathcal{F}_{2}$ e $\mathcal{F}_{3}$, de acordo com a base e a fibra de $\xi_{M}$, e fazemos os 
reconhecimentos dos elementos de $\mathcal{F}_{1}$ e $\mathcal{F}_{2}$, bem como de seus revestimentos duplos, caso existam.

No Capítulo 4, provamos o seguinte resultado: sejam $M \in \mathcal{F}$, $\operatorname{com} \xi_{M}=(E, B, F, p)$ um fibrado correspondente a $M, 0 \neq[\varphi] \in H^{1}\left(M ; \mathbb{Z}_{2}\right)$ e $\left(E_{\varphi}, q\right)$ o revestimento duplo de $E$ (associado a $\varphi)$. Se $(p \circ q)_{\#}$ não é sobrejetor, então a propriedade de BorsukUlam não vale para $\left(M_{\varphi}, \tau_{\varphi} ; \mathbb{R}^{n}\right)$, quando $n=3,4$ (Teorema 4.3). Daí, analisamos a propriedade de Borsuk-Ulam para os revestimentos duplos dos elementos das subfamílias $\mathcal{F}_{1}$ e $\mathcal{F}_{2}$, ou seja, verificamos a validade da propriedade de Borsuk-Ulam para triplas do tipo $\left(M_{\varphi}, \tau_{\varphi} ; \mathbb{R}^{n}\right)$, com $M \in \mathcal{F}_{i}, i=1,2$. Por exemplo, se $\xi_{M}=\left(E, T^{2}, \mathbb{R} P^{2}, p\right)$ é trivial, então $\pi_{1}(M) \cong \pi_{1}(E)=\left\langle a_{1}, b_{1}, c \mid\left[a_{1}, b_{1}\right], c^{2},\left[a_{1}, c\right],\left[b_{1}, c\right]\right\rangle$, e daí obtemos que se $0 \neq[\varphi] \in H^{1}\left(M ; \mathbb{Z}_{2}\right)$ é tal que $\varphi(c)=\varphi(x)=\overline{1}$, para $x=a_{1}$, ou $x=b_{1}$, então a propriedade de Borsuk-Ulam vale para $\left(M_{\varphi}, \tau_{\varphi} ; \mathbb{R}^{n}\right)$, para $n=2,3$, e não vale para $\left(M_{\varphi}, \tau_{\varphi} ; \mathbb{R}^{4}\right)$ - Proposições 4.10 (ii) e 4.12 .

Em [19], Hillman apresenta várias caracterizações para que uma 4-variedade fechada $M$ pertença à família $\mathcal{F}$. No entanto, a caracterização para que uma 4 -variedade pertença à subfamília $\mathcal{F}_{3}$, cujo fibrado correspondente tem a propriedade de que a base e a fibra sejam $K(\pi, 1)$, não foi suficiente para estudarmos os revestimentos duplos de elementos desta subfamília. Assim, a fim de obtermos alguns elementos desta subfamília equipados com involuções livres, no Capítulo 5, estudamos as chamadas variedades "flat". Entendemos por um grupo de n-variedade "flat", um grupo $\pi$ livre de torção que tem um subgrupo normal, de índice finito, isomorfo a $\mathbb{Z}^{n}$. Estas são condições necessárias e suficientes para que $\pi$ seja o grupo fundamental de uma $n$-variedade fechada "flat". São conhecidos todos os grupos de 4-variedade "flat" e eles são apresentados em [18]. Logo, são conhecidas todas as 4-variedades fechadas que são revestidas finitamente por $T^{4}$. Assim, neste capítulo, apresentamos aquelas que são revestidas duplamente por $T^{4}$, detectamos as involuções livres $\tau$ sobre $T^{4}$ e estudamos a propriedade de Borsuk-Ulam para $\left(T^{4}, \tau ; \mathbb{R}^{n}\right)$.

$\mathrm{Na}$ busca de estudarmos a propriedade de Borsuk-Ulam para mais elementos de $\mathcal{F}_{3}$ equipados com involuções livres, no Capítulo 6, consideramos os revestimentos duplos da 4-variedade quociente $\frac{K b \times K b}{\tau \times \beta}$, para determinadas involuções livres $\tau$ e $\beta$ sobre $K b$.

Mudando um pouco a dinâmica do trabalho, motivados pelo estudo da teoria de nível e conível, apresentada em [7], no Capítulo 7, apresentamos uma condição necessária e suficiente já conhecida, para a validade da propriedade de Borsuk-Ulam para $(X, \tau ; Y)$ em termos de existência de uma aplicação equivariante, mais precisamente, obtemos que 
a propriedade de Borsuk-Ulam vale para $(X, \tau ; Y)$ se, e somente se, não existe aplicação equivariante de $(X, \tau)$ em $Y^{*}$ (Proposição 7.1), onde $Y^{*}:=Y \times Y-\Delta$ ( $\Delta$ é a diagonal de $Y \times Y$ ) equipado com a involução livre que troca as coordenadas. Quando $X=\mathbb{S}^{n}$, esta condição é equivalente ao cálculo do conível de $Y^{*}$. Neste sentido, pelo trabalho de Conner-Floyd [6] (mais especificamente por [6, Teorema 33.1]) e suas generalizações, fazemos algumas observações acerca da propriedade de Borsuk-Ulam para $\left(\mathbb{S}^{n}, A ; M^{k}\right)$, onde $M^{k}$ é uma variedade topológica de dimensão $k$ e $A$ é a involução antipodal sobre $\mathbb{S}^{n}$, e sob algumas hipóteses sobre $M^{k}$, determinamos o conível de $\left(M^{k}\right)^{*}$. 


\section{Capítulo 1}

\section{Preliminares}

Este capítulo tem por objetivo introduzir conceitos, resultados e notações que serão usados no decorrer deste trabalho. Por todo o texto, entenderemos aplicação como sendo aplicação contínua e espaço como sendo espaço topológico. Sempre que houver dúvida de notação, sugerimos ao leitor a consulta da lista de símbolos, onde colocamos algumas das notações usadas no texto. Com relação aos resultados da teoria de grupos, usados em todo o trabalho, sugerimos ao leitor as seguintes referências: [30, Apêndice I, Seção 6] e [23, Seção 2.3] para o método de Reidemeister-Schreier, que trata de dar uma apresentação para um subgrupo $H$ de um grupo $G(H \leq G)$ conhecendo-se a apresentação de $G$; [5], [21] e [31] para produto semidireto; e [21] para as apresentações dos produtos direto e semidireto de grupos.

\subsection{Sobre fibrados e fibrações}

Nesta seção apresentamos algumas propriedades da teoria de fibrados, cujos detalhes poderão ser encontrados em: [4], [20], [22], 32] e [33]. Sendo $E, B$ e $F$ espaços e $p: E \rightarrow B$ uma aplicação, denotamos por $\xi=(E, B, F, p)$ um fibrado (ou $F$-fibrado sobre $B$ ) com base $B$, fibra $F$, espaço total $E$ e projeção $p$. Observemos que se $B$ é paracompacta e Hausdorff, então todo fibrado sobre $B$ é uma fibração ([32, Corolário 14, pág. 96]).

Exemplo 1.1. Consideremos $S$ uma superfície fechada (compacta e sem bordo) e $\phi: S \rightarrow S$ um homeomorfismo. Definamos $E_{\phi}=\frac{I \times S}{(0, x) \sim(1, \phi(x))}$ juntamente com uma aplicação $p: E_{\phi} \rightarrow \mathbb{S}^{1}$ dada por $p(\overline{(t, x)})=\bar{t}$, para todo $(t, x) \in I \times S, \operatorname{com} \overline{(t, x)} \in E_{\phi} \mathrm{e}$ $\bar{t} \in \frac{I}{0 \sim 1}$ denotando as classes contendo $(t, x)$ e $t$, respectivamente. Assim, $\left(E_{\phi}, \mathbb{S}^{1}, S, p\right)$ 
é um fibrado e, como $\mathbb{S}^{1}$ é compacta e Hausdorff, $p$ é uma fibração. E mais, sabemos que os $S$-fibrados sobre $\mathbb{S}^{1}$ são classificados, a menos de equivalência de fibrados, pelas classes de conjugação das classes de isotopia dos auto-homeomorfismos de $S$ que preservam ponto base ([15, Seção 1.2]). Sendo assim, temos, por exemplo, apenas dois $\mathbb{S}^{2}$-fibrados sobre $\mathbb{S}^{1}$ (onde o não trivial, é obtido pelo homeomorfismo $\phi: \mathbb{S}^{2} \rightarrow \mathbb{S}^{2}$ dado por $\phi(x)=-x$, e seu espaço total é denotado por $\left.\mathbb{S}^{1} \widetilde{\times} \mathbb{S}^{2}\right)$ e apenas um $\mathbb{R} P^{2}$-fibrado sobre $\mathbb{S}^{1}$.

Apresentamos agora algumas propriedades sobre fibrados induzidos (ou pullback) e fibrados principais.

Proposição 1.2. [22, Proposição 8.7] Sejam $\xi_{1}=\left(E_{1}, B_{1}, F_{1}, p_{1}\right)$ e $\xi_{2}=\left(E_{2}, B_{2}, F_{2}, p_{2}\right)$ dois fibrados, e $f=\left(f_{E}, f_{B}\right)$ uma aplicação fibrada de $\xi_{1}$ em $\xi_{2}$. Então, $\xi_{1}$ é equivalente ao fibrado induzido $f_{B}{ }^{*}\left(\xi_{2}\right)$.

Teorema 1.3. [22, Teorema 8.8] Sejam $\xi=(E, B, F, p)$ um fibrado e $f, g: B^{\prime} \rightarrow B$ aplicações homotópicas. Então, $f^{*}(\xi)$ e $g^{*}(\xi)$ são equivalentes.

Teorema 1.4. [22, Teorema 8.9] Qualquer fibrado sobre um espaço contrátil é equivalente ao trivial.

Teorema 1.5. [4, Cap. II, Teorema 5.8] Seja $X$ um espaço paracompacto e Hausdorff sobre o qual um grupo compacto de Lie, $G$, atua livremente. Então, $p: X \rightarrow X / G$ é um $G$-fibrado principal.

Para qualquer grupo topológico $G$, existe uma construção, denominada construção de Milnor, de um espaço contrátil $E G$, sobre o qual $G$ atua livremente, tal que o $G$-fibrado principal universal $p: E G \rightarrow E G / G=B G$, que denotamos por $\omega_{G}$, possui as seguintes propriedades:

Teorema 1.6. [20, Cap. 4, Teorema 12.2] Para qualquer $G$-fibrado principal $\xi=$ $(E, B, F, p)$, existe uma aplicação $f: B \rightarrow B G$ tal que $\xi$ e o fibrado induzido $f^{*}\left(\omega_{G}\right)$ são equivalentes.

Teorema 1.7. [20, Cap. 4, Teorema 12.4] Sejam $f_{0}, f_{1}: B \rightarrow B G$ duas aplicações tais que $f_{0}{ }^{*}\left(\omega_{G}\right)$ e $f_{1}{ }^{*}\left(\omega_{G}\right)$ são $G$-fibrados principais equivalentes sobre $B$. Então, $f_{0}$ é homotópica a $f_{1}$.

Com as hipóteses do Teorema 1.6, denominamos o espaço BG de espaço classificante e a aplicação $f$ de aplicação classificante para o $G$-fibrado principal $\xi$. 
Teorema 1.8. [32, Teorema 10, pág. 377] Dada uma fibração $p: E \rightarrow B$, com fibra sobre $b_{0} \in B, F=p^{-1}\left(b_{0}\right)$, existe uma sequência exata

$$
\cdots \longrightarrow \pi_{n}(F) \longrightarrow \pi_{n}(E) \longrightarrow \pi_{n}(B) \longrightarrow \pi_{n-1}(F) \longrightarrow \cdots \longrightarrow \pi_{0}(B),
$$

denominada sequência de homotopia da fibração.

\subsection{Espaços com involuções}

Dado um espaço $X$, uma involução sobre $X$ é um homeomorfismo $\tau: X \rightarrow X$ tal que $\tau^{2}$ é a aplicação identidade sobre $X$ (ou seja, $\left.\tau^{2}=i d_{X}\right)$. Dizemos ainda que $\tau$ é livre se ela não tem pontos fixos, isto é, $\tau(x) \neq x$, para todo $x \in X$.

Observação 1.9. A existência de uma involução livre $\tau$ sobre um espaço $X$ é equivalente à existência de uma $\mathbb{Z}_{2}$-ação livre sobre $X$. Assim, pelo Teorema 1.5, $p: X \rightarrow X / \tau$ é um $\mathbb{Z}_{2}$-fibrado principal.

Notação 1.10. Um par $(X, \tau)$ denotará, por todo o texto, um espaço $X$ equipado com uma involução livre $\tau$.

Exemplo 1.11. Consideremos a esfera $\mathbb{S}^{n}$. Temos que a aplicação $A: \mathbb{S}^{n} \rightarrow \mathbb{S}^{n}$, dada por $A(x)=-x$, é uma involução livre sobre $\mathbb{S}^{n}$, chamada de involução antipodal. A menos que digamos o contrário, sempre veremos a esfera $\mathbb{S}^{n}$ equipada com esta involução.

Definição 1.12. Dados pares $(X, \tau)$ e $(Y, \beta)$, dizemos que uma aplicação $f: X \rightarrow Y$ é equivariante (ou $\mathbb{Z}_{2}$-equivariante) se ela comuta com as involuções $\tau$ e $\beta$, isto é, $f \circ \tau=\beta \circ f$. Uma tal $f$ induz uma aplicação $\bar{f}$ entre os espaços de órbitas, $X / \tau$ e $Y / \beta$, de modo que o diagrama

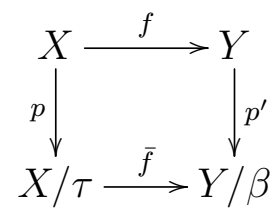

comuta.

Observação 1.13. Dado um par $(X, \tau)$, desde que $\left[X / \tau, \mathbb{R} P^{\infty}\right] \cong H^{1}\left(X / \tau ; \mathbb{Z}_{2}\right)$, então o $\mathbb{Z}_{2}$-fibrado principal $p: X \rightarrow X / \tau$ é classificado por uma classe de cohomologia $w_{X} \in$ $H^{1}\left(X / \tau ; \mathbb{Z}_{2}\right)$. Com a notação de 1.12 , a aplicação $\bar{f}$ é tal que

$$
\bar{f}^{*}\left(w_{Y}\right)=w_{X}
$$




\section{$1.3 \quad$ A propriedade de Borsuk-Ulam (PBU)}

O teorema conhecido como teorema de Borsuk-Ulam, conjecturado por Ulam e provado por Borsuk, em [3], estabelece que, para qualquer aplicação $f: \mathbb{S}^{n} \rightarrow \mathbb{R}^{n}$, existe um par de pontos antípodas $(x,-x), \operatorname{com} x \in \mathbb{S}^{n}$, de modo que $x$ e $-x$ são colapsados num mesmo ponto pela aplicação $f$, isto é, $f(x)=f(-x)$. Uma generalização natural para este teorema seria trocarmos a esfera $\mathbb{S}^{n}$ por um espaço $X$ equipado com uma involução livre $\tau$, e o espaço euclidiano $\mathbb{R}^{n}$ por um espaço $Y$, e nos perguntarmos se para toda aplicação $f: X \rightarrow Y$, existe $x \in X$ tal que $f(x)=f(\tau(x))$. Caso isto ocorra, dizemos que a terna $(X, \tau ; Y)$ satisfaz a propriedade de Borsuk-Ulam (PBU). Um de nossos objetivos neste trabalho é estudar tal propriedade para ternas do tipo $\left(X, \tau ; \mathbb{R}^{n}\right)$, onde $X$ é um CW-complexo conexo de dimensão quatro.

Sejam $X$ e $X^{\prime}$ CW-complexos quaisquer (não necessariamente de dimensão finita).

Definição 1.14. Dizemos que os pares $(X, \tau)$ e $\left(X^{\prime}, \tau^{\prime}\right)$ são equivalentes se existe um homeomorfismo $h: X \rightarrow X^{\prime}$ que é $\mathbb{Z}_{2}$-equivariante.

Observação 1.15. Se os pares $(X, \tau)$ e $\left(X^{\prime}, \tau^{\prime}\right)$ são equivalentes, então a propriedade de Borsuk-Ulam vale para $(X, \tau ; Y)$ se, e somente se, ela vale para $\left(X^{\prime}, \tau^{\prime} ; Y\right)$.

Na sequência, apresentamos as ferramentas que serão usadas no estudo da propriedade de Borsuk-Ulam para ternas do tipo $\left(X, \tau ; \mathbb{R}^{n}\right)$, cujas verificações podem ser encontradas em [13].

Proposição 1.16. [13, Proposição 2.2] Consideremos um par $(X, \tau)$. São equivalentes:

(i) a propriedade de Borsuk-Ulam vale para $\left(X, \tau ; \mathbb{R}^{n}\right)$;

(ii) não existe aplicação equivariante $f: X \rightarrow \mathbb{S}^{n-1}$;

(iii) não existe aplicação $f: X / \tau \rightarrow \mathbb{R} P^{n-1}$ tal que o pullback da classe não trivial $y \in H^{1}\left(\mathbb{R} P^{n-1} ; \mathbb{Z}_{2}\right)$ é a classe característica, $w_{X}$, do $\mathbb{Z}_{2}$-fibrado principal $p: X \rightarrow X / \tau ;$

(iv) a aplicação classificante $\gamma: X / \tau \rightarrow \mathbb{R} P^{\infty}$ não se fatora a $\mathbb{R} P^{n-1}$.

Corolário 1.17. [13, Corolário 2.3] Sejam $\tau_{1}, \tau_{2}$ duas involuções livres sobre $X$ com espaços de órbitas correspondentes $W_{1}, W_{2}$, e aplicações classificantes $\gamma_{1}, \gamma_{2}$. Suponha que as classes características representativas $w_{1} \in H^{1}\left(W_{1} ; \mathbb{Z}_{2}\right), w_{2} \in H^{1}\left(W_{2}, \mathbb{Z}_{2}\right)$ são equivalentes no sentido de que existe uma equivalência de homotopia $h: W_{1} \rightarrow W_{2}$ com $h^{*}\left(w_{2}\right)=w_{1}$. Então, a propriedade de Borsuk-Ulam vale para $\left(X, \tau_{1} ; \mathbb{R}^{n}\right)$ se, e somente se, ela vale para $\left(X, \tau_{2} ; \mathbb{R}^{n}\right)$. 
Lema 1.18. [13, Lema 2.4] Consideremos um $\operatorname{par}(X, \tau), \operatorname{com} \operatorname{dim}(X)=m<\infty$, e $n$ um inteiro positivo.

(1) Se a propriedade de Borsuk-Ulam vale para $\left(X, \tau ; \mathbb{R}^{n}\right)$, então ela também vale para $\left(X, \tau ; \mathbb{R}^{n-1}\right)$.

(2) A propriedade de Borsuk-Ulam sempre vale para $\left(X, \tau ; \mathbb{R}^{1}\right)$.

(3) A propriedade de Borsuk-Ulam nunca vale para $\left(X, \tau ; \mathbb{R}^{m+1}\right)$.

Observação 1.19. Nas hipóteses do lema anterior, nosso problema passa a ser o de encontrar o maior inteiro $n, 1 \leq n \leq \operatorname{dim}(X)$, para o qual temos a validade da propriedade de Borsuk-Ulam para a terna $\left(X, \tau ; \mathbb{R}^{n}\right)$.

Os próximos resultados nos dão critérios algébricos para o estudo da propriedade de Borsuk-Ulam.

Teorema 1.20. [13, Teorema 3.1] Consideremos um par $(X, \tau)$. A propriedade de BorsukUlam vale para $\left(X, \tau ; \mathbb{R}^{2}\right)$ se, e somente se, o homomorfismo induzido $\gamma_{\#}: \pi_{1}(X / \tau) \rightarrow$ $\pi_{1}\left(\mathbb{R} P^{\infty}\right) \cong \mathbb{Z}_{2}$ pela aplicação classificante $\gamma: X / \tau \rightarrow \mathbb{R} P^{\infty}$ não se fatora através da projeção $\mathbb{Z} \rightarrow \mathbb{Z}_{2}$, isto é, não existe homomorfismo $\eta: \pi_{1}(X / \tau) \rightarrow \pi_{1}\left(\mathbb{R} P^{1}\right) \cong \mathbb{Z}$ de modo que o seguinte diagrama comuta

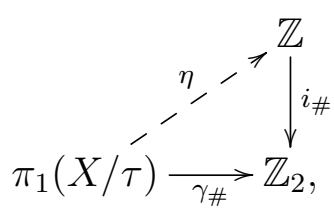

onde $i: \mathbb{R} P^{1} \rightarrow \mathbb{R} P^{\infty}$ denota a inclusão.

Como consequência deste resultado temos:

Corolário 1.21. [13, Corolário 3.3] Dado um par $(X, \tau)$, se $\pi_{1}(X / \tau)$ é finito, então a propriedade de Borsuk-Ulam vale para $\left(X, \tau ; \mathbb{R}^{2}\right)$.

Dado um par $(X, \tau)$, sendo $w \in H^{1}\left(X / \tau ; \mathbb{Z}_{2}\right)$ a classe característica do $\mathbb{Z}_{2}$-fibrado principal $X \rightarrow X / \tau$, temos:

Teorema 1.22. [13, Teorema 3.4] A propriedade de Borsuk-Ulam vale para $\left(X, \tau ; \mathbb{R}^{m}\right)$, onde $m=\operatorname{dim}(X)$, se, e somente se, $w$ satisfaz $w^{m} \neq 0$. 


\subsection{Nível e conível}

Existe um conceito intimamente relacionado com a propriedade de Borsuk-Ulam e que apresentaremos ao longo desta seção.

Definição 1.23. Seja $X$ um espaço equipado com uma involução $\tau$, não necessariamente livre. Definimos o nível e o conível de $X$, respectivamente, por

$$
\begin{aligned}
& s(X)=\inf \left\{n \mid \text { existe aplicação equivariante de } X \text { em } \mathbb{S}^{n-1}\right\} \text { e } \\
& s^{\prime}(X)=\sup \left\{m \mid \text { existe aplicação equivariante de } \mathbb{S}^{m-1} \text { em } X\right\} .
\end{aligned}
$$

Se não existe aplicação equivariante de $X$ em $\mathbb{S}^{n}$, para qualquer $n$, convencionamos $s(X)=\infty$. Se $X$ é não vazio, sempre existe aplicação equivariante de $\mathbb{S}^{0}$ em $X$, e assim $1 \leq s^{\prime}(X) \leq \infty$.

Vale observarmos que, em virtude da Proposição 1.16, verificar a validade da propriedade de Borsuk-Ulam para ternas do tipo $\left(X, \tau ; \mathbb{R}^{n}\right)$ é equivalente a determinar o nível de $X$.

O nível e o conível possuem as seguintes propriedades:

Lema 1.24. [7, Lema 2.7] Sejam $X$ e $Y$ espaços equipados com involuções. Se existe uma aplicação equivariante de $X$ em $Y$, então $s(X) \leq s(Y)$ e $s^{\prime}(X) \leq s^{\prime}(Y)$.

Lema 1.25. [7, Lema 2.8] Dado um espaço $X$ equipado com uma involução, então $s^{\prime}(X) \leq$ $s(X)$.

Exemplo 1.26. Temos que $s^{\prime}\left(\mathbb{S}^{n-1}\right)=s\left(\mathbb{S}^{n-1}\right)=n$, para todo $n \geq 2$. De fato, por um lado, da validade da propriedade de Borsuk-Ulam para $\left(\mathbb{S}^{n-1}, A ; \mathbb{R}^{k}\right)$, para todo $k \leq n-1$, obtemos que não existe aplicação equivariante de $\mathbb{S}^{n-1}$ em $\mathbb{S}^{k-1}$, com $k \leq n-1$, isto é,

$$
s\left(\mathbb{S}^{n-1}\right)>n-1 .
$$

Por outro lado, da não validade da propriedade de Borsuk-Ulam para $\left(\mathbb{S}^{n-1}, A ; \mathbb{R}^{n}\right)$, existe uma aplicação equivariante de $\mathbb{S}^{n-1}$ em $\mathbb{S}^{n-1}$, e assim

$$
s\left(\mathbb{S}^{n-1}\right) \leq n \text { e } s^{\prime}\left(\mathbb{S}^{n-1}\right) \geq n
$$

Portanto, do lema anterior juntamente com $(1.2)$ e $(1.3)$, segue que $s^{\prime}\left(\mathbb{S}^{n-1}\right)=s\left(\mathbb{S}^{n-1}\right)=$ $n$. 
Notemos que tanto o nível, quanto o conível, são interessantes para um espaço $X$ equipado com uma involução livre $\tau$. De fato, se um espaço $X$ está equipado com uma involução $\tau$ que tem pelo menos um ponto fixo, digamos $x_{0} \in X$, então, por um lado, para qualquer $n$, sempre existe uma aplicação equivariante $f: \mathbb{S}^{n-1} \rightarrow X$ (bastando definir $\left.f\left(\mathbb{S}^{n-1}\right)=x_{0}\right)$, e assim $s^{\prime}(X)=\infty$. Por outro lado, para todo $n$, não poderíamos ter uma aplicação equivariante de $X$ em $\mathbb{S}^{n-1}$, pois caso existisse uma tal $f: X \rightarrow \mathbb{S}^{n-1}$, teríamos $f\left(x_{0}\right)=f\left(\tau\left(x_{0}\right)\right)=-f\left(x_{0}\right)$, o que é uma contradição, e portanto $s(X)=\infty$.

Observação 1.27. Dado um par $(X, \tau)$ tal que a dimensão de $X$ é finita, então $s(X)$ e $s^{\prime}(X)$ são finitos. 


\section{Capítulo 2}

\section{PBU para produtos de superfícies}

\subsection{Introdução}

Em [11], Gonçalves classifica todas as involuções livres sobre uma superfície fechada $S$ qualquer e estuda a propriedade de Borsuk-Ulam para ternas do tipo $\left(S, \tau ; \mathbb{R}^{2}\right)$, obtendo o seguinte resultado:

Teorema 2.1. [11, Teorema 2.5] Seja $(S, \tau)$ um par, onde $S$ é uma superfície fechada e $\tau$ é uma $\mathbb{Z}_{2}$-ação livre sobre $S$. A propriedade de Borsuk-Ulam vale para $\left(S, \tau ; \mathbb{R}^{2}\right)$ se, e somente se, uma das seguintes condições vale:

(a) $S$ é orientável e sua característica de Euler é congruente a $2 \bmod 4$;

(b) $S$ é não orientável, sua característica de Euler é congruente a $2 \bmod 4$ e a ação $\tau$ é equivalente a uma das ações canônicas que correspondem a subgrupos dados por sequências da forma $\left(\overline{1}, \delta_{2}, \delta_{3}, \ldots, \delta_{2 r+1}\right)$, onde $\delta_{i}$ é, ou $\overline{1}$, ou $\overline{0}$;

(c) $S$ é não orientável, sua característica de Euler é congruente a $0 \bmod 4$ e a ação $\tau$ é equivalente a uma das ações canônicas que correspondem a subgrupos dados por sequências da forma $\left(\overline{1}, \delta_{2}, \delta_{3}, \ldots, \delta_{2 r}\right)$, onde $\delta_{i}$ é, ou $\overline{1}$, ou $\overline{0}$.

Observação 2.2. Cada uma das sequências do teorema acima corresponde a um epimorfismo de $\pi_{1}(S / \tau)$ em $\mathbb{Z}_{2}$, associado ao revestimento duplo $S \rightarrow S / \tau$ (maiores detalhes podem ser encontrados em [11, pág. 119]).

Motivados por [11], neste capítulo, estudamos a propriedade de Borsuk-Ulam para ternas do tipo $\left(M_{1} \times M_{2}, \tau ; \mathbb{R}^{n}\right)$, onde $M_{1}$ e $M_{2}$ são superfícies fechadas e $\tau$ é uma involução livre sobre $M_{1} \times M_{2}$ que satisfaz determinadas condições como veremos a seguir. Como $\operatorname{dim}\left(M_{1} \times M_{2}\right)=4$, então do Lema 1.18 , os casos a serem analisados são $n=2,3,4$. 


\subsection{Involução diagonal}

Dados dois espaços $X$ e $Y$, equipados com involuções $\tau$ e $\beta$, respectivamente, ambas não necessariamente livres, a aplicação $\tau \times \beta: X \times Y \rightarrow X \times Y$ definida por $(\tau \times \beta)(x, y)=$ $(\tau(x), \beta(y))$, para todo $(x, y) \in X \times Y$, é uma involução (não necessariamente livre) sobre $X \times Y$, a qual denominamos de involução diagonal. Para que ela seja livre, $\tau$ ou $\beta$ deve ser livre. Sendo assim, denotemos $\tau \times \beta$ por $\boldsymbol{\tau}_{\mathbf{1}}$, se ambas forem livres, e por $\boldsymbol{\tau}_{\mathbf{2}}$, se $\boldsymbol{\tau}$ for livre e $\boldsymbol{\beta}$ não o for. Nesta seção, estudamos a propriedade de Borsuk-Ulam para os produtos de superfícies $M_{1} \times M_{2}$, equipados, ora com $\tau_{1}$, ora com $\tau_{2}$. Existe ainda uma terceira involução diagonal livre que é aquela em que $\tau$ não é livre, mas $\beta$ o é. Entretanto, devido à simetria com $\tau_{2}$, os resultados que obtemos com tal involução são análogos aos obtidos com $\tau_{2}$. Por toda esta seção, denotaremos por $p_{1}: X \times Y \rightarrow X$ e $p_{2}: X \times Y \rightarrow Y$ as projeções na primeira e na segunda coordenada, respectivamente, que podemos verificar facilmente que são equivariantes com respeito às involuções em estudo sobre $X \times Y, X$ e $Y$.

Trabalhando com $\tau_{2}$, de uma forma geral, obtemos o seguinte resultado:

Proposição 2.3. A propriedade de Borsuk-Ulam vale para $\left(X \times Y, \tau_{2} ; \mathbb{R}^{n}\right)$ se, e somente se, ela também vale para $\left(X, \tau ; \mathbb{R}^{n}\right)$.

Demonstração. Suponhamos que a propriedade de Borsuk-Ulam não vale para $\left(X, \tau ; \mathbb{R}^{n}\right)$. Daí, pela Proposição 1.16 (equivalência (i) e (ii)), existe uma aplicação equivariante $f:(X, \tau) \rightarrow\left(\mathbb{S}^{n-1}, A\right)$. Assim, $f \circ p_{1}:\left(X \times Y, \tau_{2}\right) \rightarrow\left(\mathbb{S}^{n-1}, A\right)$ é equivariante (pois é composta de equivariantes), donde segue que a propriedade de Borsuk-Ulam não vale para $\left(X \times Y, \tau_{2} ; \mathbb{R}^{n}\right)$. Reciprocamente, se a propriedade de Borsuk-Ulam não vale para $\left(X \times Y, \tau_{2} ; \mathbb{R}^{n}\right)$, então existe uma aplicação equivariante $g:\left(X \times Y, \tau_{2}\right) \rightarrow\left(\mathbb{S}^{n-1}, A\right)$. Daí, considerando $\tau_{2}{ }^{\prime}:=\tau_{\left.2\right|_{X \times\left\{y_{0}\right\}}}$, onde $y_{0}$ é tal que $\beta\left(y_{0}\right)=y_{0}$, segue que $\tau_{2}{ }^{\prime}$ é uma involução livre sobre $X \times\left\{y_{0}\right\}$, e assim a restrição de $g$ a $X \times\left\{y_{0}\right\}$ é equivariante com respeito às involuções $\tau_{2}{ }^{\prime}$ e $A$. Como o homeomorfismo natural $h:(X, \tau) \rightarrow\left(X \times\left\{y_{0}\right\}, \tau_{2}{ }^{\prime}\right)$ é equivariante, então $g \circ h:(X, \tau) \rightarrow\left(\mathbb{S}^{n-1}, A\right)$ também o é. Portanto, a propriedade de Borsuk-Ulam não vale para $\left(X, \tau ; \mathbb{R}^{n}\right)$ (Proposição 1.16 - equivalência (i) e (ii)).

Observação 2.4. Como um caso particular da proposição anterior, temos que a propriedade de Borsuk-Ulam vale para $\left(M_{1} \times M_{2}, \tau_{2} ; \mathbb{R}^{2}\right)$ se, e somente se, ela também vale para $\left(M_{1}, \tau ; \mathbb{R}^{2}\right)$, onde $M_{1}$ e $M_{2}$ são superfícies fechadas. 
Ao considerarmos a involução $\tau_{1}$, de um modo geral, obtemos:

Proposição 2.5. Se a propriedade de Borsuk-Ulam vale para $\left(X \times Y, \tau_{1} ; \mathbb{R}^{n}\right)$, então ela também vale para $\left(X, \tau ; \mathbb{R}^{n}\right)$ e $\left(Y, \beta ; \mathbb{R}^{n}\right)$.

Demonstração. Análoga à primeira parte da prova da proposição anterior.

\section{Observação 2.6.}

(1) Se $X$ e $Y$ são CW-complexos tais que $\operatorname{dim}(X)=m$ e $\operatorname{dim}(Y)=n$, com $m, n<$ $\infty$, então pela proposição anterior, a propriedade de Borsuk-Ulam não vale para $(X \times$ $\left.Y, \tau_{1} ; \mathbb{R}^{k}\right)$, para todo $k>\operatorname{mín}\{m, n\}$.

(2) Resultados semelhantes às Proposições 2.3 e 2.5 podem ser encontrados em [34, Corolário 2.3 e Observação].

De agora em diante, nesta seção, trabalharemos com a involução livre $\tau_{1}$ sobre produtos de superfícies fechadas, $M_{1} \times M_{2}$, a fim de darmos condições necessárias e suficientes para que a propriedade de Borsuk-Ulam valha para $\left(M_{1} \times M_{2}, \tau_{1} ; \mathbb{R}^{2}\right)$. Com o intuito de simplificar a escrita, adotaremos as seguintes notações:

$$
W:=\left(M_{1} \times M_{2}\right) / \tau_{1}, W_{1}:=M_{1} / \tau \text { e } W_{2}:=M_{2} / \beta
$$

Inicialmente, vejamos o caso em que $M_{1}$, ou $M_{2}$, é a esfera $\mathbb{S}^{2}$. Sem perda de generalidade, consideremos $M_{1}=\mathbb{S}^{2}$. Como a esfera $\mathbb{S}^{2}$ é simplesmente conexa, a propriedade de Borsuk-Ulam vale para $\left(\mathbb{S}^{2}, \tau ; \mathbb{R}^{2}\right)$, para toda involução livre $\tau$ sobre $\mathbb{S}^{2}$ (Corolário 1.21). Assim, obtemos o seguinte resultado:

Teorema 2.7. A propriedade de Borsuk-Ulam vale para $\left(\mathbb{S}^{2} \times M_{2}, \tau_{1} ; \mathbb{R}^{2}\right)$ se, e somente se, ela também vale para $\left(M_{2}, \beta ; \mathbb{R}^{2}\right)$.

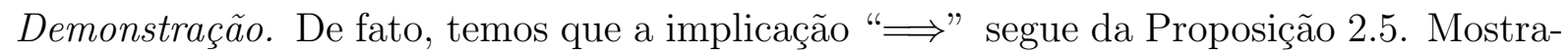
remos então a recíproca. No caso em que $M_{2}=\mathbb{S}^{2}$, como $\mathbb{S}^{2} \times \mathbb{S}^{2}$ é simplesmente conexo, o resultado segue do Corolário 1.21. Agora, seja $M_{2}$ uma superfície fechada, com característica de Euler menor ou igual a zero, e consideremos os $\mathbb{Z}_{2}$-fibrados principais $p: \mathbb{S}^{2} \times M_{2} \rightarrow W$ e $p^{\prime}: M_{2} \rightarrow W_{2}$. Como $p_{2}:\left(\mathbb{S}^{2} \times M_{2}, \tau_{1}\right) \rightarrow\left(M_{2}, \beta\right)$ é equivariante, existe $\overline{p_{2}}$, aplicação induzida entre os espaços de órbitas $W$ e $W_{2}$, tal que o diagrama

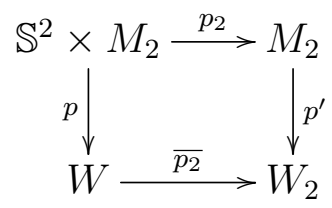


comuta. Daí, da sequência de homotopia da fibração, ora para $p$, ora para $p^{\prime}$, temos o seguinte diagrama comutativo

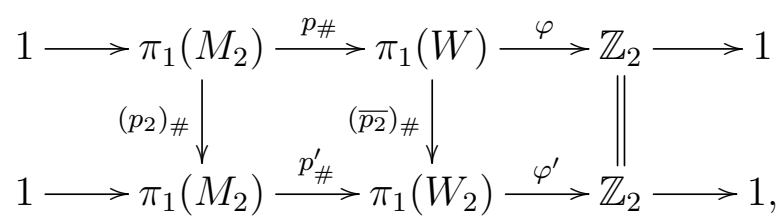

onde $\left(p_{2}\right)_{\#}$ é um isomorfismo e $\varphi=\varphi^{\prime} \circ\left(\overline{p_{2}}\right)_{\#}$. Então, pelo Lema dos Cinco, $\left(\overline{p_{2}}\right)_{\#}$ é um isomorfismo. Logo, $\varphi$ se fatora através da projeção $i_{\#}: \mathbb{Z} \rightarrow \mathbb{Z}_{2}$ (diagrama $(*$ ), do Teorema 1.20 se, e somente se, $\varphi^{\prime}$ se fatora. De fato, se existe $\eta^{\prime}: \pi_{1}\left(W_{2}\right) \rightarrow \mathbb{Z}$ tal que $i_{\#} \circ \eta^{\prime}=\varphi^{\prime}$, consideremos $\eta=\eta^{\prime} \circ\left(\overline{p_{2}}\right)_{\#}$. Assim, $i_{\#} \circ \eta=\varphi$. Reciprocamente, se existe $\eta: \pi_{1}(W) \rightarrow \mathbb{Z}$ tal que $i_{\#} \circ \eta=\varphi$, consideremos $\eta^{\prime}=\eta \circ\left(\overline{p_{2}}\right)_{\#}^{-1}$. Logo, $i_{\#} \circ \eta^{\prime}=\varphi^{\prime}$. Portanto, como a propriedade de Borsuk-Ulam vale para $\left(M_{2}, \beta ; \mathbb{R}^{2}\right)$, ou seja, como $\varphi^{\prime}$ não se fatora através da projeção $i_{\#}$, segue que $\varphi$ também não se fatora, e assim a propriedade de Borsuk-Ulam vale para $\left(\mathbb{S}^{2} \times M_{2}, \tau_{1} ; \mathbb{R}^{2}\right)$.

O próximo resultado que obtemos trata de verificar os casos em que $M_{1}$ e $M_{2}$ não são a esfera $\mathbb{S}^{2}$.

Teorema 2.8. Sejam $M_{1}$ e $M_{2}$ superfícies fechadas diferentes da esfera $\mathbb{S}^{2}$. A propriedade de Borsuk-Ulam vale para $\left(M_{1} \times M_{2}, \tau_{1} ; \mathbb{R}^{2}\right)$ se, e somente se, ela também vale para $\left(M_{1}, \tau ; \mathbb{R}^{2}\right)$ e $\left(M_{2}, \beta ; \mathbb{R}^{2}\right)$.

Demonstração. Para provarmos que a propriedade de Borsuk-Ulam vale para $\left(M_{1} \times M_{2}, \tau_{1} ; \mathbb{R}^{2}\right)$, analisaremos a fatoração do diagrama (*) (Teorema 1.20 ), e para isto, encontraremos uma apresentação para $\pi_{1}(W)$, bem como o epimorfismo $\varphi:=\gamma_{\#}$ : $\pi_{1}(W) \rightarrow \mathbb{Z}_{2}$. Sendo assim, consideremos o $\mathbb{Z}_{2}$-fibrado principal $p: M_{1} \times M_{2} \rightarrow W$ e definamos $p^{\prime}: W \rightarrow W_{1} \times W_{2}$ dado por $p^{\prime}(\overline{(x, y)})=(\bar{x}, \bar{y})$, que é um $\mathbb{Z}_{2}$-fibrado principal. De fato, definamos $\theta: \mathbb{Z}_{2} \times W \rightarrow W$ por

$$
\theta(\overline{0}, \overline{(x, y)})=\overline{0} \cdot \overline{(x, y)}=\overline{(x, y)} \text { e } \theta(\overline{1}, \overline{(x, y)})=\overline{1} \cdot \overline{(x, y)}=\overline{(\tau(x), y)} .
$$

A aplicação $\theta$ está bem definida, é uma $\mathbb{Z}_{2}$-ação livre e $W / \mathbb{Z}_{2}=W_{1} \times W_{2}$. Notemos que as involuções livres $\tau$ e $\beta$ induzem uma $\left(\mathbb{Z}_{2} \oplus \mathbb{Z}_{2}\right)$-ação livre sobre $M_{1} \times M_{2}$, de modo que $\frac{M_{1} \times M_{2}}{\mathbb{Z}_{2} \oplus \mathbb{Z}_{2}}=W_{1} \times W_{2}$. Desta forma, denotemos por $p^{\prime \prime}: M_{1} \times M_{2} \rightarrow \frac{M_{1} \times M_{2}}{\mathbb{Z}_{2} \oplus \mathbb{Z}_{2}}$ a projeção 
canônica. Daí, obtemos o seguinte diagrama (geométrico) comutativo

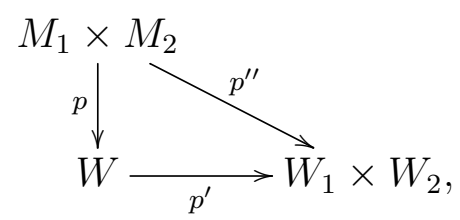

donde segue o seguinte diagrama (algébrico) comutativo

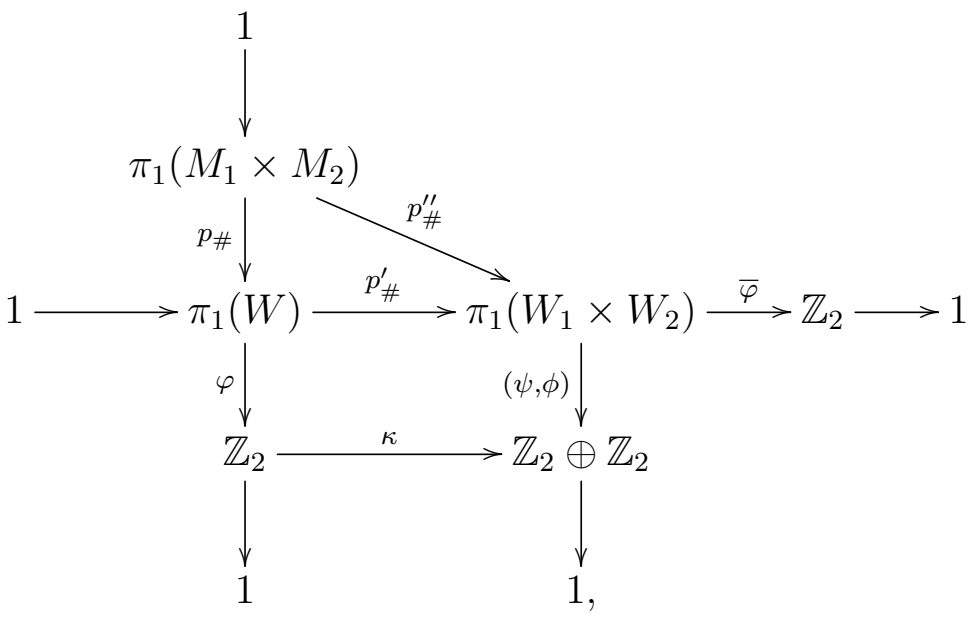

em que a aplicação $\kappa: \mathbb{Z}_{2} \rightarrow \mathbb{Z}_{2} \oplus \mathbb{Z}_{2}$ é tal que $\kappa(\overline{1})=(\overline{1}, \overline{1})$, $\psi$ é o epimorfismo de $\pi_{1}\left(W_{1}\right)$ em $\mathbb{Z}_{2}$ associado à involução $\tau$, e $\phi$ é o epimorfismo de $\pi_{1}\left(W_{2}\right)$ em $\mathbb{Z}_{2}$ associado à involução $\beta$. Como $\pi_{1}(W)=\operatorname{Ker}(\bar{\varphi})$ é um subgrupo de índice 2 em $\pi_{1}\left(W_{1} \times W_{2}\right)$, usaremos o método de Reidemeister-Schreier para determinarmos uma apresentação para o mesmo. Para isto, precisamos conhecer o epimorfismo $\bar{\varphi}$. Sendo assim, definamos $\omega: \mathbb{Z}_{2} \oplus \mathbb{Z}_{2} \rightarrow \mathbb{Z}_{2}$ por $\omega(\bar{a}, \bar{b})=\bar{a}+\bar{b}, \operatorname{com} \bar{a}, \bar{b} \in \mathbb{Z}_{2}$. Então,

$$
\bar{\varphi}=\omega \circ(\psi, \phi)
$$

De fato, uma vez que tanto o contradomínio de $\bar{\varphi}$, quanto o de $\omega \circ(\psi, \phi)$ é $\mathbb{Z}_{2}$, basta verificarmos que $\operatorname{Im}\left(p_{\#}^{\prime}\right)=\operatorname{Ker}(\omega \circ(\psi, \phi))$. Como $(\psi, \phi) \circ p_{\#}^{\prime}=\kappa \circ \varphi$, e $\varphi$ é sobrejetor, então $(\psi, \phi)$ envia os elementos de $\pi_{1}(W)$, vistos como elementos de $\pi_{1}\left(W_{1} \times W_{2}\right)$, em, ou $(\overline{0}, \overline{0})$ ou $(\overline{1}, \overline{1})$, e portanto $\operatorname{Im}\left(p_{\#}^{\prime}\right) \subset \operatorname{Ker}(\omega \circ(\psi, \phi))$. Agora, se $x \in \operatorname{Ker}(\omega \circ(\psi, \phi))$, então, pela definição de $\omega,(\psi, \phi)(x) \in\{(\overline{0}, \overline{0}),(\overline{1}, \overline{1})\}$. Por um lado, se $(\psi, \phi)(x)=(\overline{0}, \overline{0})$, então $x \in \operatorname{Ker}(\psi, \phi)=\operatorname{Im}\left(p_{\#}^{\prime \prime}\right)$, e como $p_{\#}^{\prime \prime}$ é injetor, existe um único $z \in \pi_{1}\left(M_{1} \times M_{2}\right)$ tal que $p_{\#}^{\prime \prime}(z)=x$, o que implica em $\left(p_{\#}^{\prime} \circ p_{\#}\right)(z)=x$, isto é, $x \in \operatorname{Im}\left(p_{\#}^{\prime}\right)$. Por outro lado, se $(\psi, \phi)(x)=(\overline{1}, \overline{1})=\kappa(\overline{1})$, então, da sobrejetividade de $\varphi$, existe $y \in \pi_{1}(W)$ tal que $\varphi(y)=\overline{1}$. Logo,

$$
(\psi, \phi)(x)=\kappa(\varphi(y))=(\psi, \phi)\left(p_{\#}^{\prime}(y)\right) \Rightarrow(\psi, \phi)\left(x \cdot\left(p_{\#}^{\prime}(y)\right)^{-1}\right)=(\overline{0}, \overline{0})
$$


isto é, $x \cdot\left(p_{\#}^{\prime}(y)\right)^{-1} \in \operatorname{Ker}(\psi, \phi)=\operatorname{Im}\left(p_{\#}^{\prime \prime}\right)$. Então, existe um único $z \in \pi_{1}\left(M_{1} \times M_{2}\right)$ de modo que

$$
p_{\#}^{\prime}\left(p_{\#}(z)\right)=p_{\#}^{\prime \prime}(z)=x \cdot\left(p_{\#}^{\prime}(y)\right)^{-1},
$$

donde concluímos que $p_{\#}^{\prime}\left(p_{\#}(z) \cdot y\right)=x$, isto é, $x \in \operatorname{Im}\left(p_{\#}^{\prime}\right)$. Portanto, $\bar{\varphi}=\omega \circ(\psi, \phi)$. Uma vez que temos muitos epimorfismos da forma $(\psi, \phi)$, no que se segue, faremos uma redução dos casos a serem analisados. Sejam $\psi^{\prime}$ e $\phi^{\prime}$ epimorfismos de $\pi_{1}\left(M_{1} / \tau^{\prime}\right)$ e $\pi_{1}\left(M_{2} / \beta^{\prime}\right)$ em $\mathbb{Z}_{2}$, respectivamente, associados às involuções livres $\tau^{\prime}$ e $\beta^{\prime}$ sobre $M_{1}$ e $M_{2}$, respectivamente. Como $M_{1} / \tau^{\prime} \approx W_{1}$ e $M_{2} / \beta^{\prime} \approx W_{2}$, uma vez que as características de Euler dessas variedades quocientes são ímpares, dizemos que $(\psi, \phi)$ e $\left(\psi^{\prime}, \phi^{\prime}\right)$ são relacionados, $(\psi, \phi) \sim\left(\psi^{\prime}, \phi^{\prime}\right)$, se existem isomorfismos $\xi: \pi_{1}\left(W_{1}\right) \rightarrow \pi_{1}\left(W_{1}\right)$ e $\zeta: \pi_{1}\left(W_{2}\right) \rightarrow \pi_{1}\left(W_{2}\right)$ tais que $\left(\psi^{\prime}, \phi^{\prime}\right)=(\psi \circ \xi, \phi \circ \zeta)$. É fácil ver que esta relação é de equivalência. Denote$\operatorname{mos} \frac{M_{1} \times M_{2}}{\tau^{\prime} \times \beta^{\prime}}$ por $W^{\prime}$ e seja $\varphi^{\prime}$ o epimorfismo de $\pi_{1}\left(W^{\prime}\right)$ em $\mathbb{Z}_{2}$ associado ao $\mathbb{Z}_{2}$-fibrado principal $M_{1} \times M_{2} \rightarrow W^{\prime}$.

Afirmação. Se $(\psi, \phi) \sim\left(\psi^{\prime}, \phi^{\prime}\right)$, então $\varphi$ se fatora através da projeção $i_{\#}$ (diagrama $(*)$ do Teorema 1.20 se, e somente se, $\varphi^{\prime}$ também se fatora.

Para não deixar a notação carregada, usaremos os objetos algébricos $\psi, \phi, \psi^{\prime}, \phi^{\prime}, \xi, \zeta$ também no argumento geométrico. Assim, temos o seguinte diagrama (geométrico):

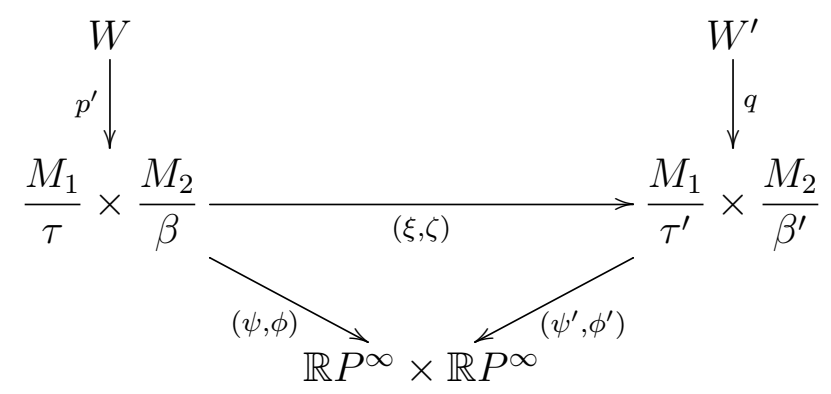

donde vem o diagrama (algébrico) abaixo:

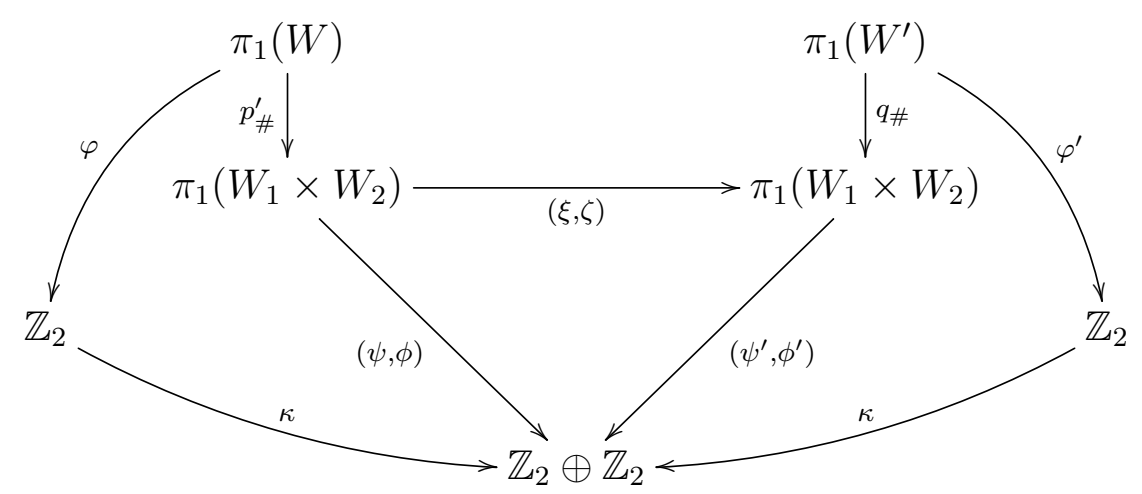

Se $x \in \pi_{1}(W)$, então, como vimos anteriormente, $(\psi, \phi)\left(p_{\#}^{\prime}(x)\right) \in\{(\overline{0}, \overline{0}),(\overline{1}, \overline{1})\}$. Agora, como o triângulo (diagrama acima) é comutativo, temos que $\left(\left(\psi^{\prime}, \phi^{\prime}\right) \circ(\xi, \zeta)\right)\left(p_{\#}^{\prime}(x)\right) \in$ 
$\{(\overline{0}, \overline{0}),(\overline{1}, \overline{1})\}$, e isto implica que $(\xi, \zeta)\left(p_{\#}^{\prime}(x)\right) \in q_{\#}\left(\pi_{1}\left(W^{\prime}\right)\right)$. Portanto, $(\xi, \zeta) \circ p^{\prime}$ se levanta a uma aplicação $\nu: W \rightarrow W^{\prime}\left(\left[24\right.\right.$, Cap. V, Teorema 5.1]), isto é, $q \circ \nu=(\xi, \zeta) \circ p^{\prime}$, de modo que $\nu_{\#}: \pi_{1}(W) \rightarrow \pi_{1}\left(W^{\prime}\right)$ é um isomorfismo. Com efeito, seja $y \in \pi_{1}\left(W^{\prime}\right)$. Daí, existe um único $z \in \pi_{1}\left(W_{1} \times W_{2}\right)$ tal que

$$
\begin{gathered}
(\xi, \zeta)(z)=q_{\#}(y) \mathrm{e} \\
(\psi, \phi)(z)=\left(\psi^{\prime}, \phi^{\prime}\right) \circ(\xi, \zeta)(z) \stackrel{(2.1)}{=}\left(\psi^{\prime}, \phi^{\prime}\right) \circ q_{\#}(y) \in\{(\overline{0}, \overline{0}),(\overline{1}, \overline{1})\},
\end{gathered}
$$

ou seja, $z \in p_{\#}^{\prime}\left(\pi_{1}(W)\right)$, donde, da injetividade de $p_{\#}^{\prime}$, consideramos $z \in \pi_{1}(W)$. Assim,

$$
\left(q_{\#} \circ \nu_{\#}\right)(z)=(\xi, \zeta)(z) \stackrel{(2.1)}{=} q_{\#}(y)
$$

Como $q_{\#}$ é monomorfismo, então $\nu_{\#}(z)=y$, e portanto $\nu_{\#}$ é um isomorfismo. Além disso, $\varphi^{\prime} \circ \nu_{\#}=\varphi$, pois

$$
\begin{aligned}
x \in \operatorname{Ker}(\varphi) & \Leftrightarrow \varphi(x)=\overline{0} \Leftrightarrow(\kappa \circ \varphi)(x)=(\overline{0}, \overline{0}) \Leftrightarrow(\psi, \phi)\left(p_{\#}^{\prime}(x)\right)=(\overline{0}, \overline{0}) \\
& \Leftrightarrow\left(\left(\psi^{\prime}, \phi^{\prime}\right) \circ(\xi, \zeta) \circ p_{\#}^{\prime}\right)(x)=(\overline{0}, \overline{0}) \Leftrightarrow\left(\left(\psi^{\prime}, \phi^{\prime}\right) \circ q_{\#} \circ \nu_{\#}\right)(x)=(\overline{0}, \overline{0}) \\
& \Leftrightarrow\left(\kappa \circ \varphi^{\prime} \circ \nu_{\#}\right)(x)=(\overline{0}, \overline{0}) \Leftrightarrow\left(\varphi^{\prime} \circ \nu_{\#}\right)(x)=\overline{0} \\
& \Leftrightarrow x \in \operatorname{Ker}\left(\varphi^{\prime} \circ \nu_{\#}\right) .
\end{aligned}
$$

Assim, temos a verificação da afirmação. Portanto, de acordo com [12, Apêndice A] e pela relação dada anteriormente, temos seis casos a serem analisados, conforme veremos na sequência.

Caso 1: Sejam $M_{1}$ e $M_{2}$ superfícies orientáveis com $\chi\left(M_{1}\right)$ e $\chi\left(M_{2}\right)$ congruentes a $2 \bmod 4$, isto é, $\chi\left(M_{1}\right)=2-4 n$ e $\chi\left(M_{2}\right)=2-4 m, \operatorname{com} n$ e $m$ inteiros maiores ou iguais a zero. Logo, $W_{1}$ (respectivamente, $W_{2}$ ) é a soma conexa de um plano projetivo e $n$ (respectivamente, m) toros, ou seja,

$$
\begin{aligned}
& \pi_{1}\left(W_{1}\right)=\left\langle u, a_{1}, a_{2}, \ldots, a_{2 n-1}, a_{2 n} \mid u^{2}\left[a_{1}, a_{2}\right] \cdots\left[a_{2 n-1}, a_{2 n}\right]\right\rangle, \\
& \pi_{1}\left(W_{2}\right)=\left\langle\widetilde{u}, \widetilde{a}_{1}, \widetilde{a}_{2} \ldots, \widetilde{a}_{2 m-1}, \widetilde{a}_{2 m} \mid \widetilde{u}^{2}\left[\widetilde{a}_{1}, \widetilde{a}_{2}\right] \cdots\left[\widetilde{a}_{2 m-1}, \widetilde{a}_{2 m}\right]\right\rangle .
\end{aligned}
$$

Neste caso, $\psi$ e $\phi$ são únicos e tais que $\psi(u)=\overline{1}, \psi\left(a_{i}\right)=\overline{0}$, para todo $1 \leq i \leq 2 n, \phi(\widetilde{u})=\overline{1}$ e $\phi\left(\widetilde{a}_{j}\right)=\overline{0}$, para todo $1 \leq j \leq 2 m$. Por [21, Cap. 4, Proposição 4], $\pi_{1}\left(W_{1} \times W_{2}\right)$ tem como geradores: $u, a_{i}, \widetilde{u}, \widetilde{a}_{j}$; e como relações: $u^{2}\left[a_{1}, a_{2}\right] \cdots\left[a_{2 n-1}, a_{2 n}\right], \widetilde{u}^{2}\left[\widetilde{a}_{1}, \widetilde{a}_{2}\right] \cdots\left[\widetilde{a}_{2 m-1}, \widetilde{a}_{2 m}\right]$, $[u, \widetilde{u}],\left[a_{i}, \widetilde{u}\right],\left[u, \widetilde{a}_{j}\right]$ e $\left[a_{i}, \widetilde{a}_{j}\right]$, com $1 \leq i \leq 2 n$ e $1 \leq j \leq 2 m$. Agora $\bar{\varphi}(u)=(\omega \circ(\psi, \phi))(u, 1)=\omega(\overline{1}, \overline{0})=\overline{1}$, 
$\bar{\varphi}\left(a_{i}\right)=(\omega \circ(\psi, \phi))\left(a_{i}, 1\right)=\omega(\overline{0}, \overline{0})=\overline{0}$,

$\bar{\varphi}(\widetilde{u})=(\omega \circ(\psi, \phi))(1, \widetilde{u})=\omega(\overline{1}, \overline{0})=\overline{1}$,

$\bar{\varphi}\left(\widetilde{a}_{j}\right)=(\omega \circ(\psi, \phi))\left(1, \widetilde{a}_{j}\right)=\omega(\overline{0}, \overline{0})=\overline{0}$,

com $1 \leq i \leq 2 n$ e $1 \leq j \leq 2 m$. Consideremos $\{1, u\}$ um sistema de Schreier. Daí, por [23, Seção 2.3], temos os seguintes geradores para $\pi_{1}(W)$ :

$y_{02}=\varrho(u, u)=u u \cdot(\overline{u u})^{-1}=u^{2}$,
$y_{i 1}=\varrho\left(1, a_{i}\right)=a_{i} \cdot\left(\overline{a_{i}}\right)^{-1}=a_{i}$,
$y_{i 2}=\varrho\left(u, a_{i}\right)=u a_{i} \cdot\left(\overline{u a_{i}}\right)^{-1}=u a_{i} u^{-1}$,
$\widetilde{y}_{01}=\varrho(1, \widetilde{u})=\widetilde{u} \cdot(\overline{\widetilde{u}})^{-1}=\widetilde{u} u^{-1}$,
$\widetilde{y}_{02}=\varrho(u, \widetilde{u})=u \widetilde{u} \cdot(\overline{u \widetilde{u}})^{-1}=u \widetilde{u}$,
$\widetilde{y}_{j 1}=\varrho\left(1, \widetilde{a}_{j}\right)=\widetilde{a}_{j} \cdot\left(\overline{\widetilde{a}_{j}}\right)^{-1}=\widetilde{a}_{j}$,
$\widetilde{y}_{j 2}=\varrho\left(u, \widetilde{a}_{j}\right)=u \widetilde{a}_{j} \cdot\left(\overline{u \widetilde{a}_{j}}\right)^{-1}=u \widetilde{a}_{j} u^{-1} ;$

e as seguintes relações:

$y_{02}\left[y_{11}, y_{21}\right] \cdots\left[y_{(2 n-1) 1}, y_{(2 n) 1}\right]=1$,

$y_{02}\left[y_{12}, y_{22}\right] \cdots\left[y_{(2 n-1) 2}, y_{(2 n) 2}\right]=1$,

$\widetilde{y}_{01} \widetilde{y}_{02}\left[\widetilde{y}_{11}, \widetilde{y}_{21}\right] \cdots\left[\widetilde{y}_{(2 m-1) 1}, \widetilde{y}_{(2 m) 1}\right]=1$,

$\widetilde{y}_{02} \widetilde{y}_{01}\left[\widetilde{y}_{12}, \widetilde{y}_{22}\right] \cdots\left[\widetilde{y}_{(2 m-1) 2}, \widetilde{y}_{(2 m) 2}\right]=1$,

$\widetilde{y}_{02} y_{02}^{-1} \widetilde{y}_{01}^{-1}=1$,

$y_{02} \widetilde{y}_{01} \widetilde{y}_{02}^{-1}=1$,

$\widetilde{y}_{j 2} \widetilde{y}_{j 1}^{-1}=1$

$y_{02} \widetilde{y}_{j 1} y_{02}^{-1} \widetilde{y}_{j 2}^{-1}=1$,

$y_{i 1} \widetilde{y}_{01} y_{i 2}^{-1} \widetilde{y}_{01}^{-1}=1$,

$y_{i 2} \widetilde{y}_{02} y_{i 1}^{-1} \widetilde{y}_{02}^{-1}=1$,

$\left[y_{i 1}, \widetilde{y}_{j 1}\right]=1$,

$\left[y_{i 2}, \widetilde{y}_{j 2}\right]=1$,

com $1 \leq i \leq 2 n$ e $1 \leq j \leq 2 m$. Logo, como $\varphi: \pi_{1}(W) \rightarrow \mathbb{Z}_{2}$ é tal que $\varphi(x)$ é a pré-imagem de $(\psi, \phi)\left(p_{\#}^{\prime}(x)\right)$ pelo homomorfismo $\kappa$, temos

$$
\begin{aligned}
& \varphi\left(y_{02}\right)=\varphi\left(u^{2}\right)=\kappa^{-1}\left((\psi, \phi)\left(u^{2}, 1\right)\right)=\kappa^{-1}(\overline{0}, \overline{0})=\overline{0} \\
& \varphi\left(y_{i 1}\right)=\varphi\left(a_{i}\right)=\kappa^{-1}\left((\psi, \phi)\left(a_{i}, 1\right)\right)=\kappa^{-1}(\overline{0}, \overline{0})=\overline{0} \\
& \varphi\left(y_{i 2}\right)=\varphi\left(u a_{i} u^{-1}\right)=\kappa^{-1}\left((\psi, \phi)\left(u a_{i} u^{-1}, 1\right)\right)=\kappa^{-1}(\overline{0}, \overline{0})=\overline{0} \\
& \varphi\left(\widetilde{y}_{01}\right)=\varphi\left(\widetilde{u} u^{-1}\right)=\kappa^{-1}\left((\psi, \phi)\left(u^{-1}, \widetilde{u}\right)\right)=\kappa^{-1}(\overline{1}, \overline{1})=\overline{1},
\end{aligned}
$$


$\varphi\left(\widetilde{y}_{02}\right)=\varphi(u \widetilde{u})=\kappa^{-1}((\psi, \phi)(u, \widetilde{u}))=\kappa^{-1}(\overline{1}, \overline{1})=\overline{1}$,

$\varphi\left(\widetilde{y}_{j 1}\right)=\varphi\left(\widetilde{a}_{j}\right)=\kappa^{-1}\left((\psi, \phi)\left(1, \widetilde{a}_{j}\right)\right)=\kappa^{-1}(\overline{0}, \overline{0})=\overline{0}$,

$\varphi\left(\widetilde{y}_{j 2}\right)=\varphi\left(u \widetilde{a}_{j} u^{-1}\right)=\kappa^{-1}\left((\psi, \phi)\left(1, \widetilde{a}_{j}\right)\right)=\kappa^{-1}(\overline{0}, \overline{0})=\overline{0}$,

com $1 \leq i \leq 2 n$ e $1 \leq j \leq 2 m$. Assim, se temos a fatoração do diagrama $(*), \eta\left(\widetilde{y}_{01}\right)$ e $\eta\left(\widetilde{y}_{02}\right)$ devem ser inteiros ímpares. No entanto, das relações (2.4), 2.6) e (2.8), segue, respectivamente, que $\eta\left(y_{02}\right)=0, \eta\left(\widetilde{y}_{01}\right)=-\eta\left(\widetilde{y}_{02}\right)$ e $\eta\left(\widetilde{y}_{01}\right)=\eta\left(\widetilde{y}_{02}\right)$, donde obtemos $\eta\left(\widetilde{y}_{01}\right)=\eta\left(\widetilde{y}_{02}\right)=0$, o que é uma contradição.

Caso 2: Sejam $M_{1}$ uma superfície orientável e $M_{2}$ uma superfície não orientável, com $\chi\left(M_{1}\right)$ e $\chi\left(M_{2}\right)$ congruentes a $2 \bmod 4$, isto é, $\chi\left(M_{1}\right)=2-4 n$ e $\chi\left(M_{2}\right)=2-4 m$, com $n, m$ inteiros maiores ou iguais a zero. Logo, $\pi_{1}\left(W_{1}\right)$ e $\pi_{1}\left(W_{2}\right)$ são como em 2.2) e (2.3), respectivamente, e $\pi_{1}\left(W_{1} \times W_{2}\right)$ tem como geradores: $u, a_{i}, \widetilde{u}, \widetilde{a}_{j}$; e como relações: $u^{2}\left[a_{1}, a_{2}\right] \cdots\left[a_{2 n-1}, a_{2 n}\right], \widetilde{u}^{2}\left[\widetilde{a}_{1}, \widetilde{a}_{2}\right] \cdots\left[\widetilde{a}_{2 m-1}, \widetilde{a}_{2 m}\right],[u, \widetilde{u}],\left[a_{i}, \widetilde{u}\right],\left[u, \widetilde{a}_{j}\right]$ e $\left[a_{i}, \widetilde{a}_{j}\right]$, com $1 \leq$ $i \leq 2 n$ e $1 \leq j \leq 2 m$. Neste caso, $\psi(u)=\overline{1}$ e $\psi\left(a_{i}\right)=\overline{0}$, com $1 \leq i \leq 2 n$, e $\phi$ é tal que $\phi(\widetilde{u})=\overline{1}$ e $\phi\left(\widetilde{a}_{j}\right)=\overline{1}$, para pelo menos um $j, 1 \leq j \leq 2 m$. Mas, de acordo com [12, Proposição $32(\mathrm{a})]$, basta considerarmos o caso em que $\phi(\widetilde{u})=\phi\left(\widetilde{a}_{1}\right)=\overline{1}$ e $\phi\left(\widetilde{a}_{j}\right)=\overline{0}$, para todo $1<j \leq 2 m$. Procedendo como no caso anterior, temos $\bar{\varphi}(u)=\bar{\varphi}(\widetilde{u})=\bar{\varphi}\left(\widetilde{a}_{1}\right)=\overline{1} \mathrm{e}$ $\bar{\varphi}\left(a_{i}\right)=\bar{\varphi}\left(\widetilde{a}_{j}\right)=\overline{0}$, com $1 \leq i \leq 2 n$ e $1<j \leq 2 m$. Então, consideremos $\{1, u\}$ um sistema de Schreier. Daí, $\pi_{1}(W)$ tem os seguintes geradores: $y_{02}=u^{2}, y_{i 1}=a_{i}, y_{i 2}=u a_{i} u^{-1}$, $\widetilde{y}_{01}=\widetilde{u} u^{-1}, \widetilde{y}_{02}=u \widetilde{u}, \widetilde{y}_{11}=\widetilde{a}_{1} u^{-1}, \widetilde{y}_{12}=u \widetilde{a}_{1}, \widetilde{y}_{j 1}=\widetilde{a}_{j}, \widetilde{y}_{j 2}=u \widetilde{a}_{j} u^{-1}$, com $1 \leq i \leq 2 n \mathrm{e}$ $1<j \leq 2 m$; e de suas relações, destacamos:

$y_{02}\left[y_{11}, y_{21}\right] \cdots\left[y_{(2 n-1) 1}, y_{(2 n) 1}\right]=1$,

$\widetilde{y}_{01} \widetilde{y}_{02} \widetilde{y}_{11} \widetilde{y}_{22} \widetilde{y}_{11}^{-1} \widetilde{y}_{21}^{-1}\left[y_{31}, y_{41}\right] \cdots\left[y_{(2 m-1) 1}, y_{(2 m) 1}\right]=1$,

$\widetilde{y}_{02} y_{02}^{-1} \widetilde{y}_{01}^{-1}=1$,

$y_{02} \widetilde{y}_{j 1} y_{02}^{-1} \widetilde{y}_{j 2}^{-1}=1$

com $1<j \leq 2 m$. Notemos que $\varphi$ é tal que $\varphi\left(y_{02}\right)=\varphi\left(y_{i 1}\right)=\varphi\left(y_{i 2}\right)=\varphi\left(\widetilde{y}_{j 1}\right)=\varphi\left(\widetilde{y}_{j 2}\right)=\overline{0}$ e $\varphi\left(\widetilde{y}_{01}\right)=\varphi\left(\widetilde{y}_{02}\right)=\varphi\left(\widetilde{y}_{11}\right)=\varphi\left(\widetilde{y}_{12}\right)=\overline{1}$. Assim, se o diagrama $(*)$ se fatora, $\eta$ deve enviar $\widetilde{y}_{01}, \widetilde{y}_{02}, \widetilde{y}_{11}$ e $\widetilde{y}_{12}$ em inteiros ímpares. No entanto, de $(2.16)$, segue que $\eta\left(y_{02}\right)=0$, e, de (2.18), segue que $\eta\left(\widetilde{y}_{01}\right)=\eta\left(\widetilde{y}_{02}\right)$. Agora, por 2.19), para $j=2$, temos $\eta\left(\widetilde{y}_{21}\right)=\eta\left(\widetilde{y}_{22}\right)$, donde, por (2.17), $\eta\left(\widetilde{y}_{01}\right)=-\eta\left(\widetilde{y}_{02}\right)$. Logo, $\eta\left(\widetilde{y}_{01}\right)=\eta\left(\widetilde{y}_{02}\right)=0$, o que é uma contradição.

Caso 3: Seja $M_{1}$ uma superfície orientável com $\chi\left(M_{1}\right)$ congruente a $2 \bmod 4$, e seja $M_{2}$ uma superfície não orientável com $\chi\left(M_{2}\right)$ congruente a $0 \bmod 4$, isto é, $\chi\left(M_{1}\right)=2-4 n$ e $\chi\left(M_{2}\right)=-4 m$, com $n, m$ inteiros maiores ou iguais a zero. Logo, $W_{1}$ é como nos casos 
anteriores, enquanto que $W_{2}$ é a soma conexa de uma garrafa de Klein e $m$ toros. Daí, $\pi_{1}\left(W_{1}\right)$ é como em (2.2), e

$$
\pi_{1}\left(W_{2}\right)=\left\langle\widetilde{u}, \widetilde{v}, \widetilde{a}_{1}, \widetilde{a}_{2}, \ldots, \widetilde{a}_{2 m-1}, \widetilde{a}_{2 m} \mid[\widetilde{u}, \widetilde{v}]^{\prime}\left[\widetilde{a}_{1}, \widetilde{a}_{2}\right] \cdots\left[\widetilde{a}_{2 m-1}, \widetilde{a}_{2 m}\right]\right\rangle .
$$

Então, $\pi_{1}\left(W_{1} \times W_{2}\right)$ tem $u, a_{i}, \widetilde{u}, \widetilde{v}, \widetilde{a}_{j}$ como geradores, e $u^{2}\left[a_{1}, a_{2}\right] \cdots\left[a_{2 n-1}, a_{2 n}\right]$, $[\widetilde{u}, \widetilde{v}]^{\prime}\left[\widetilde{a}_{1}, \widetilde{a}_{2}\right] \cdots\left[\widetilde{a}_{2 m-1}, \widetilde{a}_{2 m}\right],[u, \widetilde{u}],[u, \widetilde{v}],\left[u, \widetilde{a}_{j}\right],\left[a_{i}, \widetilde{u}\right],\left[a_{i}, \widetilde{v}\right],\left[a_{i}, \widetilde{a}_{j}\right]$ como relações, com $1 \leq i \leq 2 n$ e $1 \leq j \leq 2 m$. Como no caso $1, \psi(u)=\overline{1}$ e $\psi\left(a_{i}\right)=\overline{0}$, com $1 \leq i \leq 2 n$, e de acordo com [12, Proposição 32(b)], basta considerarmos o caso em que $\phi(\widetilde{u})=\overline{1}$ e $\phi(\widetilde{v})=\phi\left(\widetilde{a}_{j}\right)=\overline{0}, \operatorname{com} 1 \leq j \leq 2 m$. Logo, $\bar{\varphi}(u)=\bar{\varphi}(\widetilde{u})=\overline{1}$ e $\bar{\varphi}\left(a_{i}\right)=\bar{\varphi}(\widetilde{v})=\bar{\varphi}\left(\widetilde{a}_{j}\right)=\overline{0}$, com $1 \leq i \leq 2 n$ e $1 \leq j \leq 2 m$. Consideremos $\{1, u\}$ um sistema de Schreier. Daí, $\pi_{1}(W)$ possui os seguintes geradores: $y_{02}=u^{2}, y_{i 1}=a_{i}, y_{i 2}=u a_{i} u^{-1}, \widetilde{y}_{01}=\widetilde{u} u^{-1}, \widetilde{y}_{02}=u \widetilde{u}$, $\widetilde{z}_{01}=\widetilde{v}, \widetilde{z}_{02}=u \widetilde{v} u^{-1}, \widetilde{y}_{j 1}=\widetilde{a}_{j}$ e $\widetilde{y}_{j 2}=u \widetilde{a}_{j} u^{-1}$, com $1 \leq i \leq 2 n$ e $1 \leq j \leq 2 m$; e de suas relações, destacamos:

$y_{02}\left[y_{11}, y_{21}\right] \cdots\left[y_{(2 n-1) 1}, y_{(2 n) 1}\right]=1$,

$\widetilde{y}_{01} \widetilde{z}_{02} \widetilde{y}_{02} \widetilde{z}_{01}^{-1}\left[\widetilde{y}_{11}, \widetilde{y}_{21}\right] \cdots\left[\widetilde{y}_{(2 m-1) 1}, \widetilde{y}_{(2 m) 1}\right]=1$,

$\widetilde{y}_{02} y_{02}^{-1} \widetilde{y}_{01}^{-1}=1$,

$\widetilde{z}_{02} \widetilde{z}_{01}^{-1}=1$.

Como $\varphi$ envia $\widetilde{y}_{01}$ e $\widetilde{y}_{02}$ em $\overline{1}, \eta$ deve enviá-los em inteiros ímpares, caso $(*)$ se fatore. Mas, de (2.21), temos $\eta\left(y_{02}\right)=0$, e de 2.23$)$ e 2.24$)$, temos $\eta\left(\widetilde{y}_{01}\right)=\eta\left(\widetilde{y}_{02}\right)$ e $\eta\left(\widetilde{z}_{01}\right)=\eta\left(\widetilde{z}_{02}\right)$, respectivamente. Daí, por 2.22$), \eta\left(\widetilde{y}_{01}\right)=-\eta\left(\widetilde{y}_{02}\right)$, donde concluímos que $\eta\left(\widetilde{y}_{01}\right)=0$, o que é uma contradição.

Caso 4: Sejam $M_{1}$ e $M_{2}$ superfícies não orientáveis com $\chi\left(M_{1}\right)$ e $\chi\left(M_{2}\right)$ congruentes a $2 \bmod 4$, isto é, $\chi\left(M_{1}\right)=2-4 n$ e $\chi\left(M_{2}\right)=2-4 m$, com $n, m$ inteiros maiores ou iguais a zero. Assim, $\pi_{1}\left(W_{1}\right)$ e $\pi_{1}\left(W_{2}\right)$ são como em (2.2) e (2.3), respectivamente, e $\pi_{1}\left(W_{1} \times W_{2}\right)$ tem como geradores: $u, a_{i}, \widetilde{u}, \widetilde{a}_{j}$; e como relações: $u^{2}\left[a_{1}, a_{2}\right] \cdots\left[a_{2 n-1}, a_{2 n}\right]$, $\widetilde{u}^{2}\left[\widetilde{a}_{1}, \widetilde{a}_{2}\right] \cdots\left[\widetilde{a}_{2 m-1}, \widetilde{a}_{2 m}\right],[u, \widetilde{u}],\left[a_{i}, \widetilde{u}\right],\left[u, \widetilde{a}_{j}\right]$ e $\left[a_{i}, \widetilde{a}_{j}\right]$, com $1 \leq i \leq 2 n$ e $1 \leq j \leq 2 m$. De acordo com [12, Proposição 32(a)], $\psi(u)=\psi\left(a_{1}\right)=\overline{1}, \psi\left(a_{i}\right)=\overline{0}$, com $1<i \leq 2 n$, $\phi(\widetilde{u})=\phi\left(\widetilde{a}_{1}\right)=\overline{1}$ e $\phi\left(\widetilde{a}_{j}\right)=\overline{0}$, com $1<j \leq 2 m$, serão os únicos casos analisados. Ainda, $\bar{\varphi}(u)=\bar{\varphi}\left(a_{1}\right)=\bar{\varphi}(\widetilde{u})=\bar{\varphi}\left(\widetilde{a}_{1}\right)=\overline{1}$ e $\bar{\varphi}\left(a_{i}\right)=\bar{\varphi}\left(\widetilde{a}_{j}\right)=\overline{0}$, com $1<i \leq 2 n$ e $1<j \leq 2 m$.

Se consideramos $\left\{1, a_{1}\right\}$ um sistema de Schreier, então $\pi_{1}(W)$ tem os seguintes geradores: $y_{01}=u a_{1}^{-1}, y_{02}=a_{1} u, y_{12}=a_{1}^{2}, y_{i 1}=a_{i}, y_{i 2}=a_{1} a_{i} a_{1}^{-1}, \widetilde{y}_{01}=\widetilde{u} a_{1}^{-1}, \widetilde{y}_{02}=a_{1} \widetilde{u}$, $\widetilde{y}_{11}=\widetilde{a}_{1} a_{1}^{-1}, \widetilde{y}_{12}=a_{1} \widetilde{a}_{1}, \widetilde{y}_{j 1}=\widetilde{a}_{j}, \widetilde{y}_{j 2}=a_{1} \widetilde{a}_{j} a_{1}^{-1}$, com $1<i \leq 2 n$ e $1<j \leq 2 m$; e de suas relações, destacamos: 
$\widetilde{y}_{01} \widetilde{y}_{02} \widetilde{y}_{11} \widetilde{y}_{22} \widetilde{y}_{11}^{-1} \widetilde{y}_{21}^{-1}\left[y_{31}, y_{41}\right] \cdots\left[y_{(2 m-1) 1}, y_{(2 m) 1}\right]=1$,

$y_{01} \widetilde{y}_{02} y_{02}^{-1} \widetilde{y}_{01}^{-1}=1$

$y_{01} \widetilde{y}_{j 2} y_{02}^{-1} \widetilde{y}_{j 1}^{-1}=1$

$\widetilde{y}_{j 2} \widetilde{y}_{j 1}^{-1}=1$

com $1<j \leq 2 m$. Logo, de (2.28), obtemos $\eta\left(\widetilde{y}_{j 1}\right)=\eta\left(\widetilde{y}_{j 2}\right)$, com $1<j \leq 2 m$, e de 2.27 , temos $\eta\left(y_{01}\right)=\eta\left(y_{02}\right)$. Com isto, 2.25) e 2.26) implicam que $\eta\left(\widetilde{y}_{01}\right)=-\eta\left(\widetilde{y}_{02}\right)$ e $\eta\left(\widetilde{y}_{01}\right)=\eta\left(\widetilde{y}_{02}\right)$, respectivamente, donde $\eta\left(\widetilde{y}_{01}\right)=\eta\left(\widetilde{y}_{02}\right)=0$, o que é uma contradição, pois $\varphi$ envia $\widetilde{y}_{01}$ e $\widetilde{y}_{02}$ em $\overline{1}$.

Caso 5: Sejam $M_{1}$ e $M_{2}$ superfícies não orientáveis com $\chi\left(M_{1}\right)$ congruente a $2 \bmod 4$ e $\chi\left(M_{2}\right)$ congruente a $0 \bmod 4$, isto é, $\chi\left(M_{1}\right)=2-4 n$ e $\chi\left(M_{2}\right)=-4 m$, com $n, m$ inteiros maiores ou iguais a zero. Então, $\pi_{1}\left(W_{1}\right)$ e $\pi_{1}\left(W_{2}\right)$ são como em $(2.2)$ e 2.20), respectivamente. Assim, $\pi_{1}\left(W_{1} \times W_{2}\right)$ tem $u, a_{i}, \widetilde{u}, \widetilde{v}, \widetilde{a}_{j}$ como geradores, com $1 \leq i \leq 2 n$, $1 \leq j \leq 2 m$, e $u^{2}\left[a_{1}, a_{2}\right] \cdots\left[a_{2 n-1}, a_{2 n}\right],[\widetilde{u}, \widetilde{v}]^{\prime}\left[\widetilde{a}_{1}, \widetilde{a}_{2}\right] \cdots\left[\widetilde{a}_{2 m-1}, \widetilde{a}_{2 m}\right],[u, \widetilde{u}],[u, \widetilde{v}],\left[u, \widetilde{a}_{j}\right]$, $\left[a_{i}, \widetilde{u}\right],\left[a_{i}, \widetilde{v}\right]$ e $\left[a_{i}, \widetilde{a}_{j}\right]$ como relações, com $1 \leq i \leq 2 n, 1 \leq j \leq 2 m$. Temos $\psi(u)=\psi\left(a_{1}\right)=$ $\overline{1}, \psi\left(a_{i}\right)=\overline{0}$, com $1<i \leq 2 n, \phi(\widetilde{u})=\overline{1}, \phi(\widetilde{v})=\phi\left(\widetilde{a}_{j}\right)=\overline{0}$, com $1 \leq j \leq 2 m$. E mais, $\bar{\varphi}(u)=\bar{\varphi}\left(a_{1}\right)=\bar{\varphi}(\widetilde{u})=\overline{1}$ e $\bar{\varphi}\left(a_{i}\right)=\bar{\varphi}(\widetilde{v})=\bar{\varphi}\left(\widetilde{a}_{j}\right)=\overline{0}$, com $1<i \leq 2 n$ e $1 \leq j \leq 2 m$.

Logo, se consideramos $\left\{1, a_{1}\right\}$ um sistema de Schreier, então $\pi_{1}(W)$ tem como geradores: $y_{01}=u a_{1}^{-1}, y_{02}=a_{1} u, y_{12}=a_{1}^{2}, y_{i 1}=a_{i}, y_{i 2}=a_{1} a_{i} a_{1}^{-1}, \widetilde{y}_{01}=\widetilde{u} a_{1}^{-1}, \widetilde{y}_{02}=a_{1} \widetilde{u}, \widetilde{z}_{01}=\widetilde{v}$, $\widetilde{z}_{02}=a_{1} \widetilde{v} a_{1}^{-1}, \widetilde{y}_{j 1}=\widetilde{a}_{j}$ e $\widetilde{y}_{j 2}=a_{1} \widetilde{a}_{j} a_{1}^{-1}, 1<i \leq 2 n$ e $1 \leq j \leq 2 m$, e de suas relações, destacamos:

$\widetilde{y}_{01} \widetilde{z}_{02} \widetilde{y}_{02} \widetilde{z}_{01}^{-1}\left[\widetilde{y}_{11}, \widetilde{y}_{21}\right] \cdots\left[\widetilde{y}_{(2 m-1) 1}, \widetilde{y}_{(2 m) 1}\right]=1$,

$y_{01} \widetilde{y}_{02} y_{01}^{-1} \widetilde{y}_{01}^{-1}=1$,

$\widetilde{z}_{02} \widetilde{z}_{01}^{-1}=1$

Observemos que $\varphi\left(\widetilde{y}_{01}\right)=\varphi\left(\widetilde{y}_{02}\right)=\overline{1}$, e desta forma $\eta\left(\widetilde{y}_{01}\right)$ e $\eta\left(\widetilde{y}_{02}\right)$ devem ser inteiros ímpares, caso $(*)$ se fatore. Mas, de 2.31) e 2.29), temos $\eta\left(\widetilde{y}_{01}\right)=-\eta\left(\widetilde{y}_{02}\right)$, enquanto que, de (2.30), $\eta\left(\widetilde{y}_{01}\right)=\eta\left(\widetilde{y}_{02}\right)$, donde concluímos que $\eta\left(\widetilde{y}_{01}\right)=\eta\left(\widetilde{y}_{02}\right)=0$, o que é uma contradição.

Caso 6: Sejam $M_{1}$ e $M_{2}$ superfícies não orientáveis com $\chi\left(M_{1}\right)$ e $\chi\left(M_{2}\right)$ congruentes a $0 \bmod 4$, isto é, $\chi\left(M_{1}\right)=-4 n$ e $\chi\left(M_{2}\right)=-4 m$, com $n, m$ inteiros maiores ou iguais a zero. Desta forma,

$$
\pi_{1}\left(W_{1}\right)=\left\langle u, v, a_{1}, a_{2}, \ldots, a_{2 n-1}, a_{2 n} \mid[u, v]^{\prime}\left[a_{1}, a_{2}\right] \cdots\left[a_{2 n-1}, a_{2 n}\right]\right\rangle
$$


e $\pi_{1}\left(W_{2}\right)$ é como em 2.20). Desta forma, $\pi_{1}\left(W_{1} \times W_{2}\right)$ possui $u, v, a_{i}, \widetilde{u}, \widetilde{v}, \widetilde{a}_{j}$ como geradores, e $[u, v]^{\prime}\left[a_{1}, a_{2}\right] \cdots\left[a_{2 n-1}, a_{2 n}\right],[\widetilde{u}, \widetilde{v}]^{\prime}\left[\widetilde{a}_{1}, \widetilde{a}_{2}\right] \cdots\left[\widetilde{a}_{2 m-1}, \widetilde{a}_{2 m}\right],[u, \widetilde{u}],[u, \widetilde{v}],\left[u, \widetilde{a}_{j}\right]$, $[v, \widetilde{u}],[v, \widetilde{v}],\left[v, \widetilde{a}_{j}\right],\left[a_{i}, \widetilde{u}\right],\left[a_{i}, \widetilde{v}\right]$ e $\left[a_{i}, \widetilde{a}_{j}\right]$ como relações, com $1 \leq i \leq 2 n$ e $1 \leq j \leq 2 m$. Ainda, $\psi$ e $\phi$ são tais que $\psi(u)=\overline{1}, \psi(v)=\psi\left(a_{i}\right)=\overline{0}, \operatorname{com} 1 \leq i \leq 2 n, \phi(\widetilde{u})=\overline{1}, \phi(\widetilde{v})=$ $\phi\left(\widetilde{a}_{j}\right)=\overline{0}, \operatorname{com} 1 \leq j \leq 2 m$. Logo, $\bar{\varphi}(u)=\bar{\varphi}(\widetilde{u})=\overline{1}$ e $\bar{\varphi}(v)=\bar{\varphi}\left(a_{i}\right)=\bar{\varphi}(\widetilde{v})=\bar{\varphi}\left(\widetilde{a}_{j}\right)=\overline{0}$, com $1 \leq i \leq 2 n$ e $1 \leq j \leq 2 m$. Se consideramos $\{1, u\}$ um sistema de Schreier, então $\pi_{1}(W)$ tem os seguintes geradores: $y_{02}=u^{2}, z_{01}=v, z_{02}=u v u^{-1}, y_{i 1}=a_{i}, y_{i 2}=u a_{i} u^{-1}$, $\widetilde{y}_{01}=\widetilde{u} u^{-1}, \widetilde{y}_{02}=u \widetilde{u}, \widetilde{z}_{01}=\widetilde{v}, \widetilde{z}_{02}=u \widetilde{v} u^{-1}, \widetilde{y}_{j 1}=\widetilde{a}_{j}$ e $\widetilde{y}_{j 2}=u \widetilde{a}_{j} u^{-1}$, com $1 \leq i \leq 2 n \mathrm{e}$ $1 \leq j \leq 2 m$; e de suas relações destacamos:

$z_{02} y_{02} z_{01}^{-1}\left[y_{11}, y_{21}\right] \cdots\left[y_{(2 n-1) 1}, y_{(2 n) 1}\right]=1$,

$\widetilde{y}_{01} \widetilde{z}_{02} \widetilde{y}_{02} \widetilde{z}_{01}^{-1}\left[\widetilde{y}_{11}, \widetilde{y}_{21}\right] \cdots\left[\widetilde{y}_{(2 m-1) 1}, \widetilde{y}_{(2 m) 1}\right]=1$,

$\widetilde{y}_{02} y_{02}^{-1} \widetilde{y}_{01}^{-1}=1$,

$\widetilde{z}_{02} \widetilde{z}_{01}^{-1}=1$,

$z_{01} \widetilde{y}_{01} z_{02}^{-1} \widetilde{y}_{01}^{-1}=1$.

Neste caso, $\varphi\left(\widetilde{y}_{01}\right)=\varphi\left(\widetilde{y}_{02}\right)=\overline{1}$, e caso $(*)$ se fatore, $\eta\left(\widetilde{y}_{01}\right)$ e $\eta\left(\widetilde{y}_{02}\right)$ devem ser inteiros ímpares. No entanto, de 2.35) e (2.33), temos $\eta\left(\widetilde{y}_{01}\right)=-\eta\left(\widetilde{y}_{02}\right)$, e de (2.36), 2.32) e 2.34), obtemos $\eta\left(\widetilde{y}_{01}\right)=\eta\left(\widetilde{y}_{02}\right)$, e isto implica que $\eta\left(\widetilde{y}_{01}\right)=\eta\left(\widetilde{y}_{02}\right)=0$, o que é contradição.

Portanto, em todos os casos, o diagrama $(*)$ não se fatora, e assim a propriedade de Borsuk-Ulam vale para $\left(M_{1} \times M_{2}, \tau_{1} ; \mathbb{R}^{2}\right)$. A outra implicação segue diretamente da Proposição 2.5.

Ainda falta analisarmos a propriedade de Borsuk-Ulam para $\left(M_{1} \times M_{2}, \tau_{i} ; \mathbb{R}^{n}\right)$, quando $n=3,4$ e $i=1,2$. Para estes casos temos o seguinte resultado:

Proposição 2.9. A propriedade de Borsuk-Ulam não vale para $\left(M_{1} \times M_{2}, \tau_{i} ; \mathbb{R}^{n}\right)$, quando $n=3,4$ e $i=1,2$.

Demonstração. Para $i=1,2$, como a propriedade de Borsuk-Ulam não vale para $\left(M_{1}, \tau ; \mathbb{R}^{3}\right)$, pois $\operatorname{dim}\left(M_{1}\right)=2$ (Lema $\left.1.18(3)\right)$, então existe uma aplicação equivariante $f:\left(M_{1}, \tau\right) \rightarrow\left(\mathbb{S}^{2}, A\right)$ e assim $f \circ p_{1}:\left(M_{1} \times M_{2}, \tau_{i}\right) \rightarrow\left(\mathbb{S}^{2}, A\right)$ também é equivariante. Portanto, a propriedade de Borsuk-Ulam não vale para $\left(M_{1} \times M_{2}, \tau_{i} ; \mathbb{R}^{3}\right), i=1,2$ (Proposição 1.16- equivalência (i) e (ii)). Agora, do Lema 1.18(1), segue que a propriedade de BorsukUlam também não vale para $\left(M_{1} \times M_{2}, \tau_{i} ; \mathbb{R}^{4}\right), i=1,2$. 


\section{Observação 2.10 .}

(1) Para $i=1$, a prova da proposição anterior poderia ter sido feita de forma análoga, usando o fato de que a propriedade de Borsuk-Ulam não vale para $\left(M_{2}, \beta ; \mathbb{R}^{3}\right)$.

(2) De um modo geral, ainda não sabemos se existem outras involuções livres sobre os produtos de superfícies que não sejam da forma diagonal. No apêndice, apresentaremos cálculos feitos na tentativa de encontrarmos tais involuções sobre o produto $K b \times K b$.

(3) No caso de $\mathbb{S}^{2} \times \mathbb{S}^{2}$ admitir uma involução livre $\tau^{\prime}$, que não é da forma diagonal, sempre temos a validade da propriedade de Borsuk-Ulam para a terna $\left(\mathbb{S}^{2} \times \mathbb{S}^{2}, \tau^{\prime} ; \mathbb{R}^{2}\right)$, pois $\mathbb{S}^{2} \times \mathbb{S}^{2}$ é simplesmente conexo (Corolário 1.21). 


\section{Capítulo 3}

\section{Fibrados de superfície sobre superfície e involuções livres}

De agora em diante, por abuso de notação, chamaremos simplesmente de fibrado de superfície, um $F$-fibrado sobre $B$, em que a base $B$ e a fibra $F$ são superfícies fechadas (compactas e sem bordo). Assim, seja $M$ uma 4-variedade fechada que tem o mesmo tipo de homotopia do espaço total $E(M \simeq E)$ de algum fibrado de superfície, $\xi_{M}:=$ $(E, B, F, p)$, e seja $\mathcal{F}$ a família de todas as 4 -variedades fechadas com esta propriedade. Neste capítulo, descreveremos parte dos elementos da família $\mathcal{F}$, bem como analisaremos a existência de involuções livres sobre tais elementos.

\section{Observação 3.1 .}

(1) Os produtos de superfícies, estudados no capítulo anterior, são elementos de $\mathcal{F}$.

(2) Dado um elemento $M$ de $\mathcal{F}$, o fibrado de superfície correspondente $\xi_{M}$ pode não ser único. Por exemplo, se $\xi_{M}$ é o $F$-fibrado trivial sobre $B$, isto é, $M \simeq B \times F$, podemos olhar $\xi_{M}$ também como sendo o $F^{\prime}$-fibrado trivial sobre $B^{\prime}$, onde $F^{\prime}=B$ e $B^{\prime}=F$.

(3) Se $B, E$ e $F$ são complexos finitos e conexos, e $p: E \rightarrow B$ é uma fibração de Hurewicz, com fibra homotopicamente equivalente a $F$, então $\chi(E)=\chi(B) \chi(F)$ (onde $\chi(X)$ denota a característica de Euler de um espaço $X$ ), e a sequência (1.1) fornece uma sequência exata

$$
\cdots \longrightarrow \pi_{2}(B) \stackrel{\partial}{\longrightarrow} \pi_{1}(F) \longrightarrow \pi_{1}(M) \longrightarrow \pi_{1}(B) \longrightarrow 1
$$

tal que a imagem de $\pi_{2}(B)$ pelo homomorfismo conectante $\partial$ está contida no centro de $\pi_{1}(F)$ ([17, Corolário 2]).

(4) Por [27], fibrações de Hurewicz com base $B$ e fibra $X$ são classificadas pelas classes de 
homotopia de aplicações de $B$ no espaço classificante de Milgram $B E(X)$, onde $E(X)$ é o monóide de todas as equivalências de homotopia de autoaplicações de $X$, com a topologia compacto aberta.

\subsection{Involuções livres sobre elementos de $\mathcal{F}$}

Dado um espaço $X$, o conjunto formado por pares da forma $(X,[\varphi])$, onde $[\varphi]$ é uma classe não nula de $H^{1}\left(X ; \mathbb{Z}_{2}\right)$, está em correspondência bijetiva com o conjunto formado por pares $(Y, \tau)$, onde $\tau$ é uma involução livre sobre um dado espaço $Y$. De fato, dado um $\operatorname{par}(X,[\varphi])$, então, pelo isomorfismo $H^{1}\left(X ; \mathbb{Z}_{2}\right) \cong \operatorname{Hom}\left(\pi_{1}(X), \mathbb{Z}_{2}\right)$, olhando $\varphi$ como um epimorfismo de $\pi_{1}(X)$ em $\mathbb{Z}_{2}$, temos que $\operatorname{Ker}(\varphi)$ é um subgrupo de índice 2 em $\pi_{1}(X)$. Assim, da teoria de revestimento, existe um revestimento duplo regular $\left(X_{\varphi}, p^{\prime}\right)$ de $X$ com $\pi_{1}\left(X_{\varphi}\right)=\operatorname{Ker}(\varphi)$, o qual chamamos de revestimento duplo associado a $\varphi$, e

$$
A\left(X_{\varphi}, p^{\prime}\right) \cong \frac{\pi_{1}(X)}{\pi_{1}\left(X_{\varphi}\right)} \cong \mathbb{Z}_{2}
$$

onde $A\left(X_{\varphi}, p^{\prime}\right)$ denota o grupo de "deck transformations" de $\left(X_{\varphi}, p^{\prime}\right)$. Daí, $A\left(X_{\varphi}, p^{\prime}\right)=$ $\left\{i d, \tau_{\varphi}\right\}$, com $\tau_{\varphi}^{2}=i d, \tau_{\varphi}(x) \neq x$, para todo $x \in X$, e $X_{\varphi} / \tau_{\varphi}=X$. Portanto, conseguimos um par $(Y, \tau)$, onde $Y:=X_{\varphi}$ equipado com uma involução livre $\tau:=\tau_{\varphi}$. Agora, dado um par $(Y, \tau)$, onde $\tau$ é uma involução livre sobre $Y$, consideremos a fibração $p^{\prime}: Y \rightarrow$ $Y / \tau$. Fazendo $X:=Y / \tau$, então, da sequência de homotopia da fibração, obtemos um epimorfismo $\varphi$ de $\pi_{1}(X)$ em $\mathbb{Z}_{2}$, que corresponde à uma classe não nula de $H^{1}\left(X ; \mathbb{Z}_{2}\right)$, denotada por $[\varphi]$, e portanto temos um par $(X,[\varphi])$.

Observação 3.2. Dado um espaço $X$ e $0 \neq[\varphi] \in H^{1}\left(X ; \mathbb{Z}_{2}\right)$, de agora em diante, denotaremos por $X_{\varphi}$ o espaço de revestimento duplo de $X$ associado a $\varphi$, e por $\tau_{\varphi}$ a involução livre sobre $X_{\varphi}$ dada pela "deck transformation" $\left(X_{\varphi} / \tau_{\varphi}=X\right)$. Caso não seja mencionada uma classe não nula de $H^{1}\left(X ; \mathbb{Z}_{2}\right)$, denotaremos o revestimento duplo de $X$, associado a algum subgrupo de índice dois em $\pi_{1}(X)$, simplesmente por $\widehat{X}$.

Consideremos as seguintes "operações":

"Operação" 1. dados um espaço $X$ e um subgrupo de índice dois do grupo fundamental de $X$, considere o espaço $\widehat{X}$ associado a este subgrupo;

“Operação" 2. dado um par $(Y, \tau)$, onde $\tau$ é uma involução livre sobre o espaço $Y$, considere o espaço de órbitas $Y / \tau$. 
Caso a família $\mathcal{F}$ seja fechada em relação à estas operações (ou seja, se $M$ é um elemento de $\mathcal{F}$, então $\widehat{M}$ também o é, e se $\tau$ é uma involução livre sobre $M$, então $M / \tau$ também o é), então a determinação de todos os elementos da família $\mathcal{F}$ que admitem uma involução livre $\tau$, bem como a determinação de todas as suas involuções livres, poderá ser feita através do estudo dos revestimentos duplos de tais elementos, conforme veremos mais adiante. O problema de encontrarmos os revestimentos duplos de um espaço recai em um problema algébrico, em geral, factível. Para analisarmos o fechamento ou não de $\mathcal{F}$, em relação à estas operações, dividimos-na como abaixo:

- $\mathcal{F}_{1}$, formada por $M \in \mathcal{F}$ tal que $\xi_{M}=(E, B, F, p)$, onde $F$ e $B$ são $\mathbb{S}^{2}$ ou $\mathbb{R} P^{2}$;

- $\mathcal{F}_{2}$, formada por $M \in \mathcal{F}$ tal que $\xi_{M}=(E, B, F, p)$, onde $B=\mathbb{S}^{2}$ (ou $\mathbb{R} P^{2}$ ) e $F$ é $K(\pi, 1)$, ou $B$ é $K(\pi, 1)$ e $F=\mathbb{S}^{2}\left(\right.$ ou $\left.\mathbb{R} P^{2}\right) ;$

- $\mathcal{F}_{3}$, formada por $M \in \mathcal{F}$ tal que $\xi_{M}=(E, B, F, p)$, onde $F$ e $B$ são $K(\pi, 1)$.

Estas subfamílias gozam da seguinte propriedade:

Lema 3.3. As subfamílias $\mathcal{F}_{1}, \mathcal{F}_{2}$ e $\mathcal{F}_{3}$, definidas anteriormente, são disjuntas duas a duas.

Demonstração. Notemos que os elementos da subfamília $\mathcal{F}_{3}$ são $K(\pi, 1)$, enquanto que os das subfamílias $\mathcal{F}_{1}$ e $\mathcal{F}_{2}$ não o são. Agora, os elementos da subfamília $\mathcal{F}_{1}$ possuem característica de Euler igual a um, dois ou quatro, enquanto que os elementos da subfamília $\mathcal{F}_{2}$ possuem característica de Euler menor ou igual a zero, e isto conclui a prova.

Assim, quanto à questão do fechamento da família $\mathcal{F}$ em relação à operação 1 (respectivamente, 2), temos:

Lema 3.4. A família $\mathcal{F}$ é fechada em relação à operação 1 (respectivamente, 2), definida anteriormente, se, e somente se, as subfamílias $\mathcal{F}_{1}, \mathcal{F}_{2}$ e $\mathcal{F}_{3}$ são fechadas em relação à operação 1 (respectivamente, 2).

Demonstração. Seja $M$ um elemento de $\mathcal{F}_{1}, \mathcal{F}_{2}$ ou $\mathcal{F}_{3}$, que admite revestimento duplo $\widehat{M}$ e/ou involução livre $\tau$. Então,

- $\widehat{M}$ não é $K(\pi, 1)$ e $\chi(\widehat{M})=2$ ou 4 , se $M \in \mathcal{F}_{1}$;

- $\widehat{M}$ não é $K(\pi, 1)$ e $\chi(\widehat{M}) \leq 0$, se $M \in \mathcal{F}_{2}$;

- $\widehat{M}$ é $K(\pi, 1)$ e $\chi(\widehat{M}) \geq 0$, se $M \in \mathcal{F}_{3}$; e/ou 
- $M / \tau$ não é $K(\pi, 1)$ e $\chi(M / \tau)=1$ ou 2 , se $M \in \mathcal{F}_{1}$;

- $M / \tau$ não é $K(\pi, 1)$ e $\chi(M / \tau) \leq 0$, se $M \in \mathcal{F}_{2}$;

- $M / \tau$ é $K(\pi, 1)$ e $\chi(M / \tau) \geq 0$, se $M \in \mathcal{F}_{3}$.

Portanto, se a família $\mathcal{F}$ é fechada em relação à operação 1 (respectivamente, 2), segue que cada uma das três subfamílias também o é. Reciprocamente, se as subfamílias $\mathcal{F}_{1}$, $\mathcal{F}_{2}$ e $\mathcal{F}_{3}$ são fechadas em relação à operação 1 (respectivamente, 2), então claramente $\mathcal{F}$ também o é.

Portanto, o problema se reduz a estudarmos o fechamento de $\mathcal{F}_{1}, \mathcal{F}_{2}$ e $\mathcal{F}_{3}$ em relação às operações 1 e 2 . De um modo mais geral, temos o seguinte resultado:

Teorema 3.5. A família $\mathcal{F}$ é fechada em relação à operação 1.

Demonstração. Sejam $M \in \mathcal{F}$ e $\xi_{M}=(E, B, F, p)$ um fibrado de superfície correspondente. Consideremos $\widehat{M}$ o revestimento duplo de $M$ associado a algum subgrupo de índice dois de $\pi_{1}(M) \cong \pi_{1}(E)$, e $(\widehat{E}, q)$ o revestimento duplo de $E$ associado a este mesmo subgrupo (visto como subgrupo de índice dois em $\pi_{1}(E)$ ). Inicialmente, mostraremos que $\widehat{E}$ é o espaço total de um fibrado de superfície e, posteriormente, que $\widehat{M}$ tem o mesmo tipo de homotopia de $\widehat{E}$. Para isto, consideremos a fibração $p \circ q: \widehat{E} \rightarrow B, \operatorname{com} F^{\prime}:=(p \circ q)^{-1}\left(b_{0}\right)$, $b_{0} \in B$, conforme no seguinte diagrama:

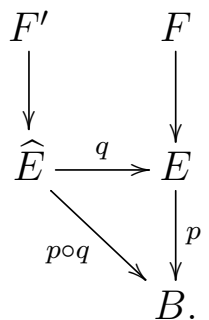

Assim, se $(p \circ q)_{\#}$ é sobrejetor, ou seja, se $F^{\prime}$ é conexa por caminhos, definamos $q^{\prime}: F^{\prime} \rightarrow F$ por $q^{\prime}(x)=q(x)$, isto é, $q^{\prime}=q_{\left.\right|_{F^{\prime}}}$. Logo,

- $q^{\prime}$ está bem definida, pois $q$ é uma aplicação fibrada;

- $q^{\prime}$ é sobrejetora. De fato, se $y \in F \subset E$, então, como $q$ é sobrejetora, existe $x \in \widehat{E}$ tal que $y=q(x)$. Daí, $(p \circ q)(x)=p(q(x))=p(y)=b_{0}$, donde segue que $x \in F^{\prime}$;

- $q^{-1}(F)=F^{\prime}$. Com efeito, se $x \in q^{-1}(F)$, então

$$
(p \circ q)(x)=p(q(x)) \stackrel{q(x) \in F}{=} b_{0},
$$

o que implica que $x \in F^{\prime}$. Agora, se $x \in F^{\prime}$, então $(p \circ q)(x)=b_{0}$, o que implica que $q(x) \in F$, donde segue que $x \in q^{-1}(F)$. 
Logo, por [32, Teorema 20, pág. 65], $q^{\prime}: F^{\prime} \rightarrow F$ é uma projeção de revestimento, com fibra discreta com dois elementos, isto é, $F^{\prime}=\widehat{F}$. Portanto, $\widehat{E}$ é o espaço total de um $\widehat{F}$-fibrado sobre $B$.

Agora, se $(p \circ q)_{\#}$ não é sobrejetor, então $(p \circ q)_{\#}\left(\pi_{1}(\widehat{E})\right)$ é um subgrupo de índice dois em $\pi_{1}(B)$, pois $q_{\#}\left(\pi_{1}(\widehat{E})\right)$ é um subgrupo de índice dois em $\pi_{1}(E)$ e $p_{\#}$ é sobrejetor. Sendo assim, consideremos $\left(\widehat{B}, q^{\prime}\right)$ o revestimento duplo de $B$ associado ao subgrupo $(p \circ q)_{\#}\left(\pi_{1}(\widehat{E})\right)$, isto é, $q_{\#}^{\prime}\left(\pi_{1}(\widehat{B})\right)=(p \circ q)_{\#}\left(\pi_{1}(\widehat{E})\right)$. Daí, $p \circ q$ se levanta a uma aplicação $p^{\prime}: \widehat{E} \rightarrow \widehat{B}$, ou seja, $q^{\prime} \circ p^{\prime}=p \circ q$. Desta forma, tomemos o fibrado induzido de $\xi_{M}$ por $q^{\prime}$, ou seja, $\left(q^{\prime}\right)^{*}\left(\xi_{M}\right)=\left(E^{*}, \widehat{B}, F, p_{2}\right)$, onde $E^{*}=\left\{(u, v) \in E \times \widehat{B} \mid p(u)=q^{\prime}(v)\right\}$ e $p_{2}(u, v)=v$, para todo $(u, v) \in E^{*}$, conforme o diagrama comutativo abaixo

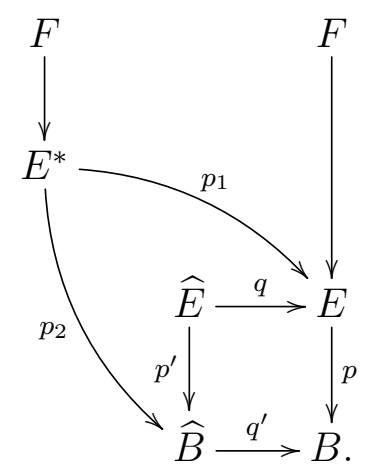

Como $\widehat{E}$ é espaço de revestimento duplo de $E$, consideremos $\widehat{\tau}$ a involução livre sobre $\widehat{E}$ dada pela "deck transformation" e definamos uma aplicação $h: \widehat{E} \rightarrow E^{*}$ por $h(x)=$ $\left(q(x), p^{\prime}(\widehat{\tau}(x))\right)$, para todo $x \in \widehat{E}$. Temos que $h$ é um homeomorfismo. De fato,

- $h$ está bem definida, pois

$$
p(q(x))=(p \circ q)(x)=(p \circ q)(\widehat{\tau}(x))=\left(q^{\prime} \circ p^{\prime}\right)(\widehat{\tau}(x))=q^{\prime}\left(p^{\prime}(\widehat{\tau}(x))\right),
$$

e é contínua;

- $h$ é sobrejetora. Com efeito, seja $(u, v) \in E^{*}$. Daí, como $q$ é sobrejetora, segue que $u=q(x)=q(\widehat{\tau}(x))$, para algum $x \in \widehat{E}$. Assim, $v=p^{\prime}(x)$ ou $p^{\prime}(\widehat{\tau}(x))$. Por um lado, se $v=p^{\prime}(x)$, então $h(\widehat{\tau}(x))=\left(q(\widehat{\tau}(x)), p^{\prime}(\widehat{\tau} \circ \widehat{\tau}(x))\right)=(u, v)$. Por outro lado, se $v=p^{\prime}(\widehat{\tau}(x))$, então $h(x)=\left(q(x), p^{\prime}(\widehat{\tau}(x))\right)=(u, v)$;

- $h$ é injetora, pois dados $x, x^{\prime} \in \widehat{E}$ tais que $x \neq x^{\prime}$, então ou $x^{\prime} \neq \widehat{\tau}(x)$ ou $x^{\prime}=\widehat{\tau}(x)$. Se $x^{\prime} \neq \widehat{\tau}(x)$, vem que $q(x) \neq q\left(x^{\prime}\right)$, e assim $h(x) \neq h\left(x^{\prime}\right)$. Se $x^{\prime}=\widehat{\tau}(x)$, então

$$
h\left(x^{\prime}\right)=\left(q(\widehat{\tau}(x)), p^{\prime}(\widehat{\tau} \circ \widehat{\tau}(x))\right)=\left(q(x), p^{\prime}(x)\right) \neq\left(q(x), p^{\prime}(\widehat{\tau}(x))\right)=h(x) .
$$

Portanto, como $\widehat{E}$ é compacto e $E^{*}$ é Hausdorff, segue que $\widehat{E}$ é o espaço total de um $F$-fibrado sobre $\widehat{B}$. 
Uma vez que $\widehat{E}$ e $\widehat{M}$ são revestimentos duplos de $E$ e $M$, respectivamente, temos

$$
\pi_{n}(\widehat{E}) \cong \pi_{n}(E) \cong \pi_{n}(M) \cong \pi_{n}(\widehat{M})
$$

para todo $n \geq 2$, e da forma como $\widehat{E}$ e $\widehat{M}$ foram considerados, temos $\pi_{1}(\widehat{E}) \cong \pi_{1}(\widehat{M})$. Portanto, $\widehat{M} \simeq \widehat{E}$.

Assim, sabemos que os revestimentos duplos dos elementos de $\mathcal{F}$ também são elementos de $\mathcal{F}$, e estão equipados com as involuções livres dadas pelas "deck transformations". Desta forma, nas próximas duas seções, na maioria dos casos, descreveremos os elementos das subfamílias $\mathcal{F}_{i}, i=1,2$, e em termos desta descrição, identificaremos seus revestimentos duplos. Também faremos algumas observações acerca do quociente de elementos destas subfamílias por uma involução livre.

\subsection{Subfamília $\mathcal{F}_{1}$}

Dividimos os fibrados que correspondem à subfamília $\mathcal{F}_{1}$ nos seguintes casos:

(1) $F=B=\mathbb{S}^{2}$,

(2) $F=\mathbb{R} P^{2}$ e $B=\mathbb{S}^{2}$,

(3) $F=\mathbb{S}^{2}$ e $B=\mathbb{R} P^{2}$,

(4) $F=B=\mathbb{R} P^{2}$

que não formam uma partição de $\mathcal{F}_{1}$.

Observemos que, se $\xi_{M}$ é como no caso (1), então $M$ não admite revestimento duplo conexo, pois $M$ é simplesmente conexa, e se $\xi_{M}$ é como no caso (4), então $M$ não admite involução livre, pois $\chi(M)=1$.

Lema 3.6. Seja $M \in \mathcal{F}_{1}$.

(i) Se $\xi_{M}$ está no caso (2), ou (3), então $\xi_{\widehat{M}}$ está no caso (1);

(ii) se $\xi_{M}$ está no caso (4), então $\xi_{\widehat{M}}$ está no caso (2), ou (3).

Demonstração. A prova segue do Teorema 3.5 e do Lema 3.4 juntamente com o fato de que $\chi(\widehat{M})=2 \chi(M)$.

Lema 3.7. Seja $(M, \tau)$ um par, onde $M \in \mathcal{F}_{1}$. Supondo que $M / \tau \in \mathcal{F}_{1}$, temos:

(i) se $\xi_{M}$ está no caso (1), então $\xi_{M / \tau}$ está no caso (2), ou (3);

(ii) se $\xi_{M}$ está no caso (2), ou (3), então $\xi_{M / \tau}$ está no caso (4). 
Demonstração. A prova segue do fato de que $\chi(M / \tau)=\chi(M) / 2$.

Observação 3.8. Não sabemos se, em geral, a subfamília $\mathcal{F}_{1}$ é fechada em relação à operação 2. Sendo assim, nas subseções a seguir, faremos o reconhecimento dos elementos de $\mathcal{F}_{1}$, bem como dos seus revestimentos duplos, caso existam, em cada um dos quatro casos.

\subsubsection{Base $\mathbb{S}^{2}$}

Como $\mathbb{S}^{2}$ é a união dos hemisférios superior $H^{+}$e inferior $H^{-}$, e um $F$-fibrado sobre $H^{+}\left(H^{-}\right)$é trivial (Teorema 1.4), então um $F$-fibrado sobre $\mathbb{S}^{2}$ é determinado, a menos de equivalência de fibrados, pela classe de homotopia da aplicação de colagem em $\left[\mathbb{S}^{1}, \operatorname{Homeo}(F)\right]=\pi_{1}(\operatorname{Homeo}(F))$.

Caso 1: $F=B=\mathbb{S}^{2}$

Como $\pi_{1}\left(\right.$ Homeo $\left.\left(\mathbb{S}^{2}\right)\right) \cong \pi_{1}(S O(3))=\mathbb{Z}_{2}\left(\left[9\right.\right.$, pág. 21]), temos dois $\mathbb{S}^{2}$-fibrados sobre $\mathbb{S}^{2}$ (a menos de equivalência de fibrados), e um modelo para o espaço total do não trivial é dado por

$$
\mathbb{S}^{2} \widetilde{\times} \mathbb{S}^{2}:=\frac{\left(H^{+} \times \mathbb{S}^{2}\right) \amalg\left(H^{-} \times \mathbb{S}^{2}\right)}{(x, y) \sim(x, \phi(x)(y))},
$$

onde $x=e^{2 \pi t i} \in \partial H^{+} \cap \partial H^{-}, t \in I$, e $\phi(x): \mathbb{S}^{2} \rightarrow \mathbb{S}^{2}$ é a rotação em torno do eixo vertical no sentido anti-horário por um ângulo $2 \pi t$. Como comentamos anteriormente, $\mathbb{S}^{2} \times \mathbb{S}^{2} \mathrm{e}$ $\mathbb{S}^{2} \widetilde{\times} \mathbb{S}^{2}$ não admitem revestimentos duplos conexos.

\section{Caso 2: $F=\mathbb{R} P^{2}$ e $B=\mathbb{S}^{2}$}

Há dois $\mathbb{R} P^{2}$-fibrados sobre $\mathbb{S}^{2}$ (a menos de equivalência de fibrados), pois

$$
\pi_{1}\left(\operatorname{Homeo}\left(\mathbb{R} P^{2}\right)\right) \cong \pi_{1}(S O(3))=\mathbb{Z}_{2}
$$

([9, pág. 21]). O espaço total do não trivial é dado por

$$
E_{[\underline{[3.3)}}:=\frac{\left(H^{+} \times \mathbb{R} P^{2}\right) \amalg\left(H^{-} \times \mathbb{R} P^{2}\right)}{(x, y) \sim(x, \phi(x)(y))},
$$

onde $x=e^{2 \pi t i} \in \partial H^{+} \cap \partial H^{-}, t \in I$, e $\phi(x): \mathbb{R} P^{2} \rightarrow \mathbb{R} P^{2}$ é a rotação em torno do eixo vertical no sentido anti-horário por um ângulo $2 \pi t$. Assim:

Proposição 3.9. Seja $\xi_{M}$ um $\mathbb{R} P^{2}$-fibrado sobre $\mathbb{S}^{2}$. Então,

(i) $\xi_{\widehat{M}}$ é o $\mathbb{S}^{2}$-fibrado trivial sobre $\mathbb{S}^{2}$, se $\xi_{M}$ é trivial;

(ii) $\xi_{\widehat{M}}$ é o $\mathbb{S}^{2}$-fibrado não trivial sobre $\mathbb{S}^{2}$, se $\xi_{M}$ é não trivial. 
Demonstração. Por [19, Teorema 5.19(5)], $\pi_{1}(M) \cong \mathbb{Z}_{2}$, e, pelo Lema 3.6, sabemos que $\xi_{\widehat{M}}$ é um $\mathbb{S}^{2}$-fibrado sobre $\mathbb{S}^{2}$. Desta forma,

(i) consideremos sobre o espaço $\mathbb{S}^{2} \times \mathbb{S}^{2}$, a involução livre dada por $\tau(x, y)=(x,-y)$. Como $\left(\mathbb{S}^{2} \times \mathbb{S}^{2}\right) / \tau=\mathbb{S}^{2} \times \mathbb{R} P^{2}$, segue que $\mathbb{S}^{2} \times \mathbb{S}^{2}$ reveste duplamente $\mathbb{S}^{2} \times \mathbb{R} P^{2}$, ou seja, $\xi_{\widehat{M}}$ é o $\mathbb{S}^{2}$-fibrado trivial sobre $\mathbb{S}^{2}$

(ii) segundo [19, pág. 238], o espaço total do $\mathbb{R} P^{2}$-fibrado não trivial sobre $\mathbb{S}^{2}$ é o quociente de $\mathbb{S}^{2} \widetilde{\times} \mathbb{S}^{2}$ por uma involução fibrada (isto é, um homeomorfismo que leva cada fibra nela mesma) que é a aplicação antipodal sobre cada fibra. Portanto, $\widehat{M} \simeq \mathbb{S}^{2} \widetilde{\times} \mathbb{S}^{2}$, ou seja, $\xi_{\widehat{M}}$ é o $\mathbb{S}^{2}$-fibrado não trivial sobre $\mathbb{S}^{2}$.

\subsubsection{Base $\mathbb{R} P^{2}$}

Como $\mathbb{R} P^{2}=\mathbb{M} \cup \mathbb{D}^{2}$ (união de uma faixa de Möbius e um disco), um $F$-fibrado sobre $\mathbb{R} P^{2}$ é determinado por um fibrado sobre $\mathbb{M}$ que restrito a $\partial \mathbb{M}$ é trivial, e pela aplicação de colagem que é um elemento de $\pi_{1}(\operatorname{Homeo}(F))$.

\section{Caso 3: $F=\mathbb{S}^{2}$ e $B=\mathbb{R} P^{2}$}

Temos quatro classes de $\mathbb{S}^{2}$-fibrados sobre $\mathbb{R} P^{2}$. De fato, notemos que são dois os $\mathbb{S}^{2}$-fibrados sobre $\mathbb{M}$, pois, por [19, Lema 5.9],

$$
\left[\mathbb{M} ; B E\left(\mathbb{S}^{2}\right)\right] \cong[\mathbb{M} ; B O(3)]=\left[\mathbb{S}^{1} ; B O(3)\right]=\pi_{1}(B O(3)) \cong \mathbb{Z}_{2}
$$

O espaço total do $\mathbb{S}^{2}$-fibrado não trivial sobre $\mathbb{M}$ é dado como segue: consideremos o círculo central $\mathbb{S}^{1}$ de $\mathbb{M}$. Pelo Exemplo 1.1, $\xi=\left(\mathbb{S}^{1} \widetilde{\times} \mathbb{S}^{2}, \mathbb{S}^{1}, \mathbb{S}^{2}, q\right)$ é o $\mathbb{S}^{2}$-fibrado não trivial sobre $\mathbb{S}^{1}$. Tomando a retração $r: \mathbb{M} \rightarrow \mathbb{S}^{1}$, o fibrado induzido $r^{*}(\xi)$ com projeção $p^{\prime}$ é o $\mathbb{S}^{2}$-fibrado não trivial sobre $\mathbb{M}$, ou seja,

$$
r^{*}\left(\mathbb{S}^{1} \widetilde{\times} \mathbb{S}^{2}\right)=\left\{(u, v) \in \mathbb{M} \times\left(\mathbb{S}^{1} \widetilde{\times} \mathbb{S}^{2}\right) \mid r(u)=q(v)\right\}
$$

é o espaço total do $\mathbb{S}^{2}$-fibrado não trivial sobre $\mathbb{M}$. Daí, para cada $x=e^{2 \pi t i} \in \partial \mathbb{M} \cap \partial \mathbb{D}^{2}$, considerando $i d(x), \phi(x) \in \operatorname{Homeo}\left(\mathbb{S}^{2}\right)$, os homeomorfismos identidade e rotação (aquela como no Caso 1), a menos de equivalência de fibrados, temos os seguintes espaços totais dos $\mathbb{S}^{2}$-fibrados não triviais sobre $\mathbb{R} P^{2}$ :

$$
\begin{aligned}
& E_{\underline{(3.5)}}:=\frac{\left(\mathbb{M} \times \mathbb{S}^{2}\right) \amalg\left(\mathbb{D}^{2} \times \mathbb{S}^{2}\right)}{(x, y) \sim(x, \phi(x)(y))}, \\
& E_{(\underline{3.6)}}:=\frac{r^{*}\left(\mathbb{S}^{1} \widetilde{\times} \mathbb{S}^{2}\right) \amalg\left(\mathbb{D}^{2} \times \mathbb{S}^{2}\right)}{(x, y) \sim(x, y)}, \\
& E_{[\text {[3.7) }}:=\frac{r^{*}\left(\mathbb{S}^{1} \widetilde{\times} \mathbb{S}^{2}\right) \amalg\left(\mathbb{D}^{2} \times \mathbb{S}^{2}\right)}{(x, y) \sim(x, \phi(x)(y))} .
\end{aligned}
$$


Proposição 3.10. Seja $\xi_{M}$ um $\mathbb{S}^{2}$-fibrado sobre $\mathbb{R} P^{2}$. Então, $\xi_{\widehat{M}}$ é o $\mathbb{S}^{2}$-fibrado trivial sobre $\mathbb{S}^{2}$.

Demonstração. Da prova do Teorema 3.5, temos o seguinte diagrama

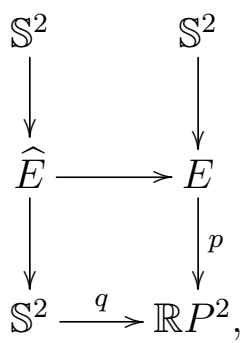

onde $\left(\mathbb{S}^{2}, q\right)$ é revestimento duplo de $\mathbb{R} P^{2}$. Notemos que

$$
q^{-1}\left(\mathbb{R} P^{2}\right)=q^{-1}\left(\mathbb{M} \cup \mathbb{D}^{2}\right)=\mathcal{C} \cup \mathbb{D}_{1}^{2} \cup \mathbb{D}_{2}^{2},
$$

onde $\mathcal{C}=q^{-1}(\mathbb{M})=\mathbb{S}^{1} \times[0,1]$ (o cilindro) e $q^{-1}\left(\mathbb{D}^{2}\right)=\mathbb{D}_{1}^{2} \cup \mathbb{D}_{2}^{2}$ (as calotas superior e inferior, respectivamente). Sobre cada uma das componentes conexas de $q^{-1}\left(\mathbb{R} P^{2}\right)$, temos os $\mathbb{S}^{2}$-fibrados triviais. No caso em que $E=\mathbb{R} P^{2} \times \mathbb{S}^{2}$ ou $E_{[3.6]}$, como o bordo de cada uma destas componentes se projeta injetivamente em $\mathbb{M} \cap \mathbb{D}^{2}$ através de $q$, segue que a colagem do bordo superior, $\mathbb{S}^{1} \times\{1\}$, de $\mathcal{C}$ com o bordo do disco $\mathbb{D}_{1}^{2}$ é dada por $i d(x) \in \operatorname{Homeo}\left(\mathbb{S}^{2}\right)$, para cada $x \in \partial \mathbb{D}_{1}^{2}$. O mesmo acontece com a colagem do bordo inferior, $\mathbb{S}^{1} \times\{0\}$, de $\mathcal{C}$ com o bordo do disco $\mathbb{D}_{2}^{2}$. Assim, $\xi_{\widehat{M}}$ é o $\mathbb{S}^{2}$-fibrado trivial sobre $\mathbb{S}^{2}$. Agora, se $E=E_{[3.5}$ ou $E_{[3.7]}$, as colagens citadas anteriormente são dadas pela rotação, ou seja,

$$
\widehat{E}=\frac{\left(\mathbb{D}_{1}^{2} \times \mathbb{S}^{2}\right) \amalg\left(\mathcal{C} \times \mathbb{S}^{2}\right) \amalg\left(\mathbb{D}_{2}^{2} \times \mathbb{S}^{2}\right)}{(x, y) \sim(x, 1, \phi(x)(y)),(z, y) \sim(z, 0, \phi(z)(y))},
$$

onde $\phi(x), \phi(z) \in \operatorname{Homeo}\left(\mathbb{S}^{2}\right)$ são dados pela rotação (aquela como no caso 1), para cada $x \in \partial \mathbb{D}_{1}^{2}, z \in \partial \mathbb{D}_{2}^{2}$ e $y \in \mathbb{S}^{2}$. Uma vez que $\mathbb{S}^{2} \times \mathbb{S}^{2}$ pode ser obtido como

$$
\frac{\left(\mathbb{D}_{1}^{2} \times \mathbb{S}^{2}\right) \amalg\left(\mathcal{C} \times \mathbb{S}^{2}\right) \amalg\left(\mathbb{D}_{2}^{2} \times \mathbb{S}^{2}\right)}{(x, y) \sim(x, 1, y),(z, y) \sim(z, 0, y)},
$$

onde $x \in \partial \mathbb{D}_{1}^{2}, z \in \partial \mathbb{D}_{2}^{2}$ e $y \in \mathbb{S}^{2}$, podemos construir um homeomorfismo entre $\widehat{E}$ e $\mathbb{S}^{2} \times \mathbb{S}^{2}$, donde segue o resultado.

Caso 4: $F=B=\mathbb{R} P^{2}$

Por [19, Lema 5.15],

$$
\left[\mathbb{M} ; B E\left(\mathbb{R} P^{2}\right)\right] \cong[\mathbb{M} ; B S O(3)]=\pi_{1}(B S O(3))=\pi_{0}(S O(3))=1,
$$


ou seja, temos apenas um $\mathbb{R} P^{2}$-fibrado sobre $\mathbb{M}$. Como $\pi_{1}\left(\operatorname{Homeo}\left(\mathbb{R} P^{2}\right)\right)=\mathbb{Z}_{2}$, temos duas classes de $\mathbb{R} P^{2}$-fibrados sobre $\mathbb{R} P^{2}$. O espaço total do $\mathbb{R} P^{2}$-fibrado não trivial sobre $\mathbb{R} P^{2}$ é dado por

$$
E_{[\underline{(3.8)}}:=\frac{\left(\mathbb{M} \times \mathbb{R} P^{2}\right) \amalg\left(\mathbb{D}^{2} \times \mathbb{R} P^{2}\right)}{(x, y) \sim(x, \phi(x)(y))},
$$

onde $\phi(x) \in \operatorname{Homeo}\left(\mathbb{R} P^{2}\right)$ é como no Caso 2, para cada $x \in \partial \mathbb{M}$. Assim, seja $\xi_{M}$ um $\mathbb{R} P^{2}$-fibrado sobre $\mathbb{R} P^{2}$. Como

$$
\pi_{1}(E) \cong \mathbb{Z}_{2} \oplus \mathbb{Z}_{2}=\left\langle a \mid a^{2}\right\rangle \times\left\langle b \mid b^{2}\right\rangle
$$

([19, pág. 238]), onde $a$ e $b$ denotam os geradores dos grupos fundamentais da base e da fibra, respectivamente, temos três espaços de revestimento duplo de $M$, associados aos epimorfismos de $\pi_{1}(M)$ em $\mathbb{Z}_{2}$ dados na seguinte tabela:

\begin{tabular}{|l|l|l|}
\hline & $\boldsymbol{a}$ & $\boldsymbol{b}$ \\
\hline $\boldsymbol{\varphi}_{\mathbf{1}}$ & $\overline{1}$ & $\overline{0}$ \\
\hline $\boldsymbol{\varphi}_{\mathbf{2}}$ & $\overline{0}$ & $\overline{1}$ \\
\hline $\boldsymbol{\varphi}_{\mathbf{3}}$ & $\overline{1}$ & $\overline{1}$ \\
\hline
\end{tabular}

Tabela 3.1: Epimorfismos de $\pi_{1}(M)$ em $\mathbb{Z}_{2}$.

Proposição 3.11. Sejam $\xi_{M}=\left(E, \mathbb{R} P^{2}, \mathbb{R} P^{2}, p\right)$ o fibrado trivial e $0 \neq[\varphi] \in H^{1}\left(M ; \mathbb{Z}_{2}\right)$. Com a notação anterior, $\xi_{M_{\varphi}}$ é o

(i) $\mathbb{R} P^{2}$-fibrado trivial sobre $\mathbb{S}^{2}$, para $\varphi=\varphi_{1}$;

(ii) $\mathbb{S}^{2}$-fibrado trivial sobre $\mathbb{R} P^{2}$, para $\varphi=\varphi_{2}$;

(iii) $\mathbb{S}^{2}$-fibrado sobre $\mathbb{R} P^{2}$ tal que $E_{\varphi}=E_{[3.6]}$, para $\varphi=\varphi_{3}$.

Demonstração. Consideremos o produto $\mathbb{S}^{2} \times \mathbb{R} P^{2}$ equipado com a involução livre $\tau$ dada por $\tau(x, y)=(-x, y)$. Logo, $\mathbb{S}^{2} \times \mathbb{R} P^{2}$ reveste duplamente $\mathbb{R} P^{2} \times \mathbb{R} P^{2}$, uma vez que $\left(\mathbb{S}^{2} \times \mathbb{R} P^{2}\right) / \tau=\mathbb{R} P^{2} \times \mathbb{R} P^{2}$. Daí, olhando $\mathbb{S}^{2} \times \mathbb{R} P^{2}$, ora como espaço total do $\mathbb{S}^{2}$-fibrado trivial sobre $\mathbb{R} P^{2}$, ora como espaço total do $\mathbb{R} P^{2}$-fibrado trivial sobre $\mathbb{S}^{2}$, da sequência de homotopia da fibração para o revestimento $\mathbb{S}^{2} \times \mathbb{R} P^{2} \rightarrow\left(\mathbb{S}^{2} \times \mathbb{R} P^{2}\right) / \tau$, temos a prova de (i) e (ii). No caso de (iii), consideremos $E_{(\underline{3.6})}$ o espaço total de um $\mathbb{S}^{2}$-fibrado sobre $\mathbb{R} P^{2}$ (dado em (3.6)), e definamos sobre $E_{[3.6]}$ uma involução livre como segue: sobre $\mathbb{D}^{2} \times \mathbb{S}^{2}$, seja $\tau_{1}$ a involução dada por $\tau_{1}(x, y)=(x,-y)$, e sobre $r^{*}\left(\mathbb{S}^{1} \widetilde{\times} \mathbb{S}^{2}\right)=\{(u, v) \in$ $\left.\mathbb{M} \times\left(\mathbb{S}^{1} \widetilde{\times} \mathbb{S}^{2}\right) \mid r(u)=q(v)\right\}\left(\right.$ como em $(3.4)$ ), seja $\tau_{2}$ dada por $\tau_{2}(u, v)=(u, \beta(v))$, (onde $\beta$ 
é a involução sobre $\mathbb{S}^{1} \widetilde{\times} \mathbb{S}^{2}=\frac{[0,1] \times \mathbb{S}^{2}}{(0, y) \sim(1,-y)}$ dada por $\beta(\overline{(t, y)})=\overline{(t,-y)}$, que está bem definida, pois

$$
\beta(\overline{(0, y)})=\overline{(0,-y)}=\overline{(1, y)}=\beta(\overline{(1,-y)})
$$

e é livre). Notemos que $\tau_{1}$ e $\tau_{2}$ coincidem em $\partial \mathbb{D}^{2} \cap \partial \mathbb{M}$, pois sobre $\partial \mathbb{M}$ temos o $\mathbb{S}^{2}$ fibrado trivial. Portanto, temos uma involução livre $\tau^{\prime}:=\tau_{1} \cup \tau_{2}$ bem definida sobre $E_{(3.6)}$. Como $\left(\mathbb{D}^{2} \times \mathbb{S}^{2}\right) / \tau_{1}=\mathbb{D}^{2} \times \mathbb{R} P^{2}, r^{*}\left(\mathbb{S}^{1} \widetilde{\times} \mathbb{S}^{2}\right) / \tau_{2}=\mathbb{M} \times \mathbb{R} P^{2}$ e a colagem em $E_{([3.6)}$ é dada pela identidade, segue que $E_{(3.6)} / \tau^{\prime}=\mathbb{R} P^{2} \times \mathbb{R} P^{2}$, ou seja, $E_{(3.6)}$ reveste duplamente $\mathbb{R} P^{2} \times \mathbb{R} P^{2}$. Como $E_{(3.6)} \not \mathbb{S}^{2} \times \mathbb{R} P^{2}$, então $E_{\varphi}=E_{(\underline{3.6)}}$ para $\varphi=\varphi_{3}$.

Proposição 3.12. Sejam $\xi_{M}=\left(E, \mathbb{R} P^{2}, \mathbb{R} P^{2}, p\right)$ o fibrado não trivial e $0 \neq[\varphi] \in$ $H^{1}\left(M ; \mathbb{Z}_{2}\right)$. Então, de acordo com a Tabela 3.1. $\xi_{M_{\varphi}}$ é o

(i) $\mathbb{R} P^{2}$-fibrado trivial sobre $\mathbb{S}^{2}$, para $\varphi=\varphi_{1}$;

(ii) $\mathbb{S}^{2}$-fibrado não trivial sobre $\mathbb{R} P^{2}$ tal que $E_{\varphi}=E_{[\underline{3.5})}$, para $\varphi=\varphi_{2}$;

(iii) $\mathbb{S}^{2}$-fibrado não trivial sobre $\mathbb{R} P^{2}$ tal que $E_{\varphi}=E_{[3.7]}$, para $\varphi=\varphi_{3}$.

Demonstração. Observemos que $E=E_{[3.8)}$ (dado em (3.8)).

(i) Da sequência de homotopia da fibração para $p$, o epimorfismo

$$
\varphi:=p_{\#}: \pi_{1}(E) \rightarrow \mathbb{Z}_{2}=\pi_{1}\left(\mathbb{R} P^{2}\right)
$$

é tal que $\varphi=\varphi_{1}$ (Tabela 3.1). Assim, considerando o revestimento duplo $\left(E_{\varphi}, p^{\prime}\right)$ de $E$, a fibração $p \circ p^{\prime}: E_{\varphi} \rightarrow \mathbb{R} P^{2}$ é tal que

$$
\left(p \circ p^{\prime}\right)_{\#}\left(\pi_{1}\left(E_{\varphi}\right)\right)=p_{\#}\left(p_{\#}^{\prime}\left(\pi_{1}\left(E_{\varphi}\right)\right)\right)=p_{\#}(\operatorname{Ker}(\varphi))=1
$$

ou seja, $\left(p \circ p^{\prime}\right)_{\#}\left(\pi_{1}\left(E_{\varphi}\right)\right)$ é um subgrupo de índice dois em $\pi_{1}\left(\mathbb{R} P^{2}\right)$. Logo, pela prova do Teorema 3.5. $\xi_{M_{\varphi}}$ é um $\mathbb{R} P^{2}$-fibrado sobre $\mathbb{S}^{2}$. Sendo $\left(\mathbb{S}^{2}, q\right)$ o revestimento duplo de $\mathbb{R} P^{2}$, temos

$$
\mathbb{S}^{2}=q^{-1}\left(\mathbb{R} P^{2}\right)=q^{-1}\left(\mathbb{M} \cup \mathbb{D}^{2}\right)=\mathcal{C} \cup \mathbb{D}_{1}^{2} \cup \mathbb{D}_{2}^{2}
$$

Daí, analogamente à prova da Proposição 3.10, segue que $\xi_{M_{\varphi}}$ é trivial.

(ii) Seja $\left(\mathbb{S}^{2}, q\right)$ o revestimento duplo de $\mathbb{R} P^{2}$ e consideremos $E_{(3.5)}$ o espaço total do $\mathbb{S}^{2}$-fibrado não trivial sobre $\mathbb{R} P^{2}$ (dado em $(3.5)$ ). Definamos $q^{\prime}: E_{(3.5)} \rightarrow E_{(3.8)}$ por $q^{\prime}(\overline{(x, y)})=\overline{(x, q(y))}$. A aplicação $q^{\prime}$ está bem definida, pois

$$
\left.q^{\prime}(\overline{(x, y)})=\overline{(x, q(y))}=\overline{(x, \phi(t)(q(y)))}=\overline{\left(x, q\left(\phi^{\prime}(t)(y)\right)\right)}=q^{\prime} \overline{\left(x, \phi^{\prime}(x)(y)\right)}\right)
$$


para $x \in \partial \mathbb{M} \cap \partial \mathbb{D}^{2}$, e é uma projeção de revestimento duplo. Da sequência de homotopia da fibração, o revestimento duplo $q^{\prime}$ está associado ao epimorfismo $\varphi_{2}$, donde segue o resultado.

(iii) Agora, seja $E_{[3.7]}$, espaço total de um $\mathbb{S}^{2}$-fibrado sobre $\mathbb{R} P^{2}$ (dado em (3.7)). Consideremos a involução livre $\tau^{\prime}$ sobre $E_{[3.7]}$, do mesmo modo que na prova da proposição anterior (item (iii)), e assim, de modo análogo, temos $E_{[3.7]} / \tau^{\prime}=E_{[3.8]}$. Portanto, segue que $E_{\varphi}=E_{(3.7)}$, para $\varphi=\varphi_{3}$.

Observação 3.13. Com o procedimento de considerarmos os revestimentos duplos de elementos de $\mathcal{F}_{1}$, então, pelos resultados anteriores, encontramos quatro involuções livres sobre $\mathbb{S}^{2} \times \mathbb{S}^{2}$, uma sobre $\mathbb{S}^{2} \widetilde{\times} \mathbb{S}^{2}$, duas sobre $\mathbb{S}^{2} \times \mathbb{R} P^{2}$ e uma sobre cada um dos espaços $E_{[3.5]}, E_{[3.6]}$ e $E_{[3.7]}$. No diagrama abaixo, expressamos os revestimentos duplos que correspondem à estas involuções.

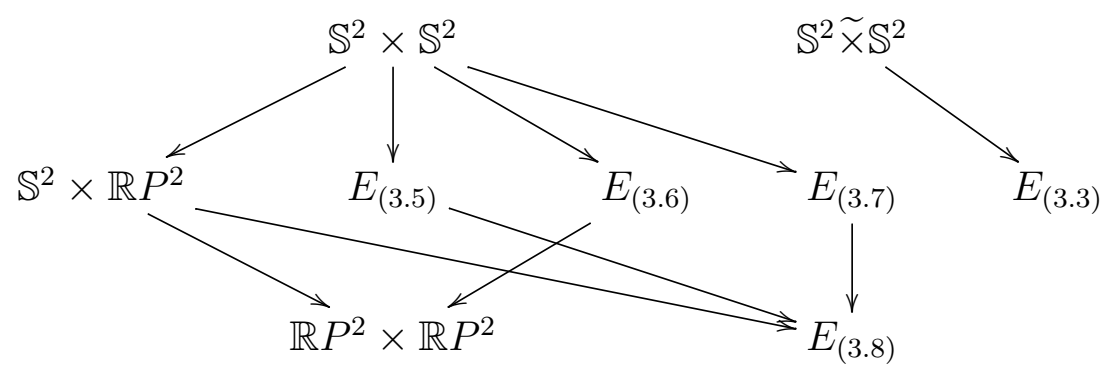

\subsection{Subfamília $\mathcal{F}_{2}$}

Nesta seção, fazemos o reconhecimento dos elementos da subfamília $\mathcal{F}_{2}$, bem como de seus revestimentos duplos. Dividimos os fibrados de superfície, $\xi_{M}=(E, B, F, p)$, que correspondem à esta subfamília nos seguintes casos:
(1) $F=\mathbb{S}^{2}$ e $\begin{cases}(\mathbf{1 a}) & \chi(B)=0 ; \\ (\mathbf{1 b}) & \chi(B)<0 ;\end{cases}$
(2) $F=\mathbb{R} P^{2}$ e $\left\{\begin{array}{l}(\mathbf{2 a}) \chi(B)=0 \\ (\mathbf{2 b}) \chi(B)<0\end{array}\right.$
(3) $B=\mathbb{S}^{2}$ e $\begin{cases}(\mathbf{3 a}) & \chi(F)=0 ; \\ (\mathbf{3 b}) & \chi(F)<0 ;\end{cases}$
(4) $B=\mathbb{R} P^{2}$ e $\begin{cases}(4 \mathbf{a}) & \chi(F)=0 ; \\ (4 \mathbf{b}) & \chi(F)<0 .\end{cases}$ 
Lema 3.14. Seja $M$ um elemento da subfamília $\mathcal{F}_{2}$.

(i) se $\xi_{M}$ está no caso (1a) (respectivamente, (1b)), então $\xi_{\widehat{M}}$ está no caso (1a) (respectivamente, $(1 \mathrm{~b}))$;

(ii) se $\xi_{M}$ está no caso (2a) (respectivamente, $(2 \mathrm{~b})$ ), então $\xi_{\widehat{M}}$ está no caso (1a), ou (2a) (respectivamente, $(1 \mathrm{~b})$, ou $(2 \mathrm{~b}))$;

(iii) se $\xi_{M}$ está no caso (3a) (respectivamente, $(3 \mathrm{~b})$ ), então $\xi_{\widehat{M}}$ está no caso (3a) (respectivamente, $(3 \mathrm{~b}))$;

(iv) se $\xi_{M}$ está no caso (4a) (respectivamente, (4b)), então $\xi_{\widehat{M}}$ está no caso (3a), ou (4a) (respectivamente, (3b), ou (4b));.

Demonstração. Como a esfera $\mathbb{S}^{2}$ não admite revestimento duplo conexo (pois é simplesmente conexa), o revestimento duplo conexo de $\mathbb{R} P^{2}$ é $\mathbb{S}^{2}$ e o revestimento duplo de uma superfície fechada $K(\pi, 1)$ com característica de Euler zero (respectivamente, menor do que zero) também é $K(\pi, 1)$ com característica de Euler zero (respectivamente, menor do que zero), o resultado segue da prova do Teorema 3.5 .

Observação 3.15. No item (i), do lema anterior, se $\xi_{\widehat{M}}$, por exemplo, é trivial, então poderíamos vê-lo também no caso (3a), ou (3b).

\subsection{1 $F=\mathbb{S}^{2}$ e $B$ é $K(\pi, 1)$}

Em [26], Melvin dá um critério para os espaços totais de $\mathbb{S}^{2}$-fibrados sobre superfícies compactas $B$ serem homeomorfos, em termos das classes de Stiefel-Whitney. Assim, quando a base é fechada, ou seja, quando temos um fibrado de superfície $\xi_{M}=\left(E, B, \mathbb{S}^{2}, p\right)$, ele é classificado pelos invariantes $w_{2}\left(\xi_{M}\right) \in\{0,1\}$ e $s\left(w_{1}\left(\xi_{M}\right)\right)$ que é igual a

- $\infty$, se $w_{1}\left(\xi_{M}\right)=w_{1}(B)$;

- 0 , se $w_{1}\left(\xi_{M}\right)=0 \neq w_{1}(B)$;

- 1, se $w_{1}\left(\xi_{M}\right) \neq 0, w_{1}\left(\xi_{M}\right) \neq w_{1}(B)$ e $w_{1}\left(\xi_{M}\right) \smile w_{1}(B)=1$;

- 2 , se $w_{1}\left(\xi_{M}\right) \neq 0, w_{1}\left(\xi_{M}\right) \neq w_{1}(B)$ e $w_{1}\left(\xi_{M}\right) \smile w_{1}(B)=0$

([26]), onde $w_{i}\left(\xi_{M}\right) \in H^{i}\left(B ; \mathbb{Z}_{2}\right)$ denota a $i$-ésima classe de Stiefel-Whitney de $\xi_{M}$. Assim,

$$
s\left(w_{1}\left(\xi_{M}\right)\right)= \begin{cases}2, \infty, & \text { se } B \text { é orientável, } \operatorname{com} \chi(B) \leq 0 \\ 0,1, \infty, & \text { se } B=K b \\ 0,1,2, \infty, & \text { se } B \text { é não orientável, } \operatorname{com} \chi(B)<0\end{cases}
$$


ou seja, a menos de equivalência de fibrados, existem

- quatro $\mathbb{S}^{2}$-fibrados sobre $B, \operatorname{com} B$ orientável e $\chi(B) \leq 0$;

- seis $\mathbb{S}^{2}$-fibrados sobre $K b$; e

- oito $\mathbb{S}^{2}$-fibrados sobre $B$, com $B$ não orientável e $\chi(B)<0$.

Observação 3.16. Sejam $\xi_{M}=\left(E, B, \mathbb{S}^{2}, p\right)$, com $B$ superfície fechada $K(\pi, 1)$, e $0 \neq$ $[\varphi] \in H^{1}\left(M ; \mathbb{Z}_{2}\right)$.

(1) Como $\pi_{1}(M) \cong \pi_{1}(B)$, podemos olhar $\varphi$ também como um epimorfismo de $\pi_{1}(B)$ em $\mathbb{Z}_{2}$

(2) da prova do Teorema 3.5. $\xi_{M_{\varphi}}=\left(E_{\varphi}, B_{\varphi}, \mathbb{S}^{2}, p^{\prime}\right)$, com $\left(E_{\varphi}, q^{\prime}\right)$ e $\left(B_{\varphi}, q\right)$ sendo revestimentos duplos de $E$ e $B$, respectivamente, associados a $\varphi$;

(3) como $\left(q^{\prime}, q\right)$ é uma aplicação fibrada, isto é, $p \circ q^{\prime}=q \circ p^{\prime}$, então

$$
w_{i}\left(\xi_{M_{\varphi}}\right)=q^{*}\left(w_{i}\left(\xi_{M}\right)\right)
$$

$\left(\left[28, \S 4\right.\right.$, Axioma 2]). Assim, $w_{2}\left(\xi_{M_{\varphi}}\right)=0$, pois $q^{*}: H^{2}\left(B ; \mathbb{Z}_{2}\right) \rightarrow H^{2}\left(B_{\varphi} ; \mathbb{Z}_{2}\right)$ é a multiplicação por 2 ([19, Lema 5.11(4)]) e

$$
w_{1}\left(\xi_{M_{\varphi}}\right)=q^{*}\left(w_{1}\left(\xi_{M}\right)\right)=w_{1}\left(\xi_{M}\right)_{\left.\right|_{\operatorname{Ker}(\varphi)}},
$$

onde a classe $w_{1}\left(\xi_{M}\right)$ é vista como um homomorfismo de $\pi_{1}(B)$ em $\mathbb{Z}_{2}$.

Analisemos os casos:

\section{(I) $B$ orientável com $\chi(B) \leq 0$}

Denominamos cada uma das quatro classes de $\mathbb{S}^{2}$-fibrados sobre $\boldsymbol{S}_{\boldsymbol{g}}$ (superfície fechada orientável de gênus $g \geq 1$ ) como sendo do tipo $\mathcal{O}_{i}, i=1, \ldots, 4$, conforme os invariantes dados anteriormente, organizados na seguinte tabela:

\begin{tabular}{|c|c|c|c|c|}
\hline & $\mathcal{O}_{\mathbf{1}}$ & $\mathcal{O}_{\mathbf{2}}$ & $\mathcal{O}_{\mathbf{3}}$ & $\mathcal{O}_{\mathbf{4}}$ \\
\hline $\boldsymbol{w}_{\mathbf{2}}\left(\boldsymbol{\xi}_{\boldsymbol{M}}\right)$ & 0 & 0 & 1 & 1 \\
\hline $\boldsymbol{s}\left(\boldsymbol{w}_{\mathbf{1}}\left(\boldsymbol{\xi}_{\boldsymbol{M}}\right)\right)$ & 2 & $\infty$ & 2 & $\infty$ \\
\hline
\end{tabular}

E denotamos por $E_{\mathcal{O}_{i}}$, com $i=1, \ldots, 4$, a menos de equivalência de fibrados, seus respectivos espaços totais. Observemos que o $\mathbb{S}^{2}$-fibrado trivial sobre $S_{g}(g \geq 1)$ corresponde ao tipo $\mathcal{O}_{2}([28, \S 4$, Proposição 2]). Adotando a apresentação

$$
\pi_{1}(M) \cong \pi_{1}\left(S_{g}\right)=\left\langle a_{1}, b_{1}, \ldots, a_{g}, b_{g} \mid\left[a_{1}, b_{1}\right] \cdots\left[a_{g}, b_{g}\right]\right\rangle,
$$


temos $2^{2 g}-1$ epimorfismos de $\pi_{1}(M)$ em $\mathbb{Z}_{2}$, e daí se $\xi_{M}$ é do tipo $\mathcal{O}_{1}$, ou $\mathcal{O}_{3}$, temos $w_{1}\left(\xi_{M}\right) \neq 0$, que vista como um epimorfismo, corresponde a um destes $2^{2 g}-1$.

Proposição 3.17. Sejam $\xi_{M}$ um $\mathbb{S}^{2}$-fibrado sobre $S_{g}(g \geq 1)$ e $0 \neq[\varphi] \in H^{1}\left(M ; \mathbb{Z}_{2}\right)$. Com a notação anterior,

(i) se $\xi_{M}$ é do tipo $\mathcal{O}_{1}$, ou $\mathcal{O}_{3}$, então $\xi_{M_{\varphi}}$ é do tipo

- $\mathcal{O}_{2}$, para $\varphi=w_{1}\left(\xi_{M}\right)$,

- $\mathcal{O}_{1}$, para $\varphi \neq w_{1}\left(\xi_{M}\right)$

(ii) se $\xi_{M}$ é do tipo $\mathcal{O}_{2}$, ou $\mathcal{O}_{4}$, então $\xi_{M_{\varphi}}$ é do tipo $\mathcal{O}_{2}$, para todo $\varphi$.

Demonstração. Como já vimos na observação anterior, $w_{2}\left(\xi_{M_{\varphi}}\right)=0$. Agora,

(i) se $\xi_{M}$ é do tipo $\mathcal{O}_{1}$, ou $\mathcal{O}_{3}$, então $w_{1}\left(\xi_{M}\right) \neq 0=w_{1}\left(S_{g}\right)$. Logo, para $\varphi=w_{1}\left(\xi_{M}\right)$,

$$
w_{1}\left(\xi_{M_{\varphi}}\right)=q^{*}\left(w_{1}\left(\xi_{M}\right)\right)=0=w_{1}\left(\left(S_{g}\right)_{\varphi}\right)\left(\text { pois }\left(S_{g}\right)_{\varphi}\right. \text { é orientável), }
$$

e portanto $s\left(w_{1}\left(\xi_{M_{\varphi}}\right)\right)=\infty$, donde segue que $\xi_{M_{\varphi}}$ é do tipo $\mathcal{O}_{2}$. Para $\varphi \neq w_{1}\left(\xi_{M}\right)$,

$$
w_{1}\left(\xi_{M_{\varphi}}\right)=q^{*}\left(w_{1}\left(\xi_{M}\right)\right) \neq 0=w_{1}\left(\left(S_{g}\right)_{\varphi}\right) \text { e } w_{1}\left(\xi_{M_{\varphi}}\right) \smile w_{1}\left(\left(S_{g}\right)_{\varphi}\right)=0 .
$$

Assim, $s\left(w_{1}\left(\xi_{M_{\varphi}}\right)\right)=2$, ou seja, $\xi_{M_{\varphi}}$ é do tipo $\mathcal{O}_{1}$.

(ii) Se $\xi_{M}$ é do tipo $\mathcal{O}_{2}$, ou $\mathcal{O}_{4}$, temos $w_{1}\left(\xi_{M}\right)=w_{1}\left(S_{g}\right)=0$. Então,

$$
w_{1}\left(\xi_{M_{\varphi}}\right)=q^{*}\left(w_{1}\left(\xi_{M}\right)\right)=0=w_{1}\left(\left(S_{g}\right)_{\varphi}\right)
$$

donde vem que $s\left(w_{1}\left(\xi_{M_{\varphi}}\right)\right)=\infty$, ou seja, $\xi_{M_{\varphi}}$ é do tipo $\mathcal{O}_{2}$, para todo $\varphi$.

\section{(II) $B$ é a garrafa de Klein}

Denominamos cada uma das seis classes de $\mathbb{S}^{2}$-fibrados sobre $K b$ como sendo do tipo $\mathcal{K}_{i}$, $i=1, \ldots, 6$, conforme os invariantes dados anteriormente, organizados na seguinte tabela:

\begin{tabular}{|c|c|c|c|c|c|c|}
\hline & $\mathcal{K}_{\mathbf{1}}$ & $\mathcal{K}_{\mathbf{2}}$ & $\mathcal{K}_{\boldsymbol{3}}$ & $\mathcal{K}_{\boldsymbol{4}}$ & $\mathcal{K}_{\boldsymbol{5}}$ & $\mathcal{K}_{\boldsymbol{6}}$ \\
\hline $\boldsymbol{w}_{\mathbf{2}}\left(\boldsymbol{\xi}_{M}\right)$ & 0 & 0 & 0 & 1 & 1 & 1 \\
\hline $\boldsymbol{s}\left(\boldsymbol{w}_{\mathbf{1}}\left(\boldsymbol{\xi}_{M}\right)\right)$ & 0 & 1 & $\infty$ & 0 & 1 & $\infty$ \\
\hline
\end{tabular}

E denotamos por $E_{\mathcal{K}_{i}}$, com $i=1, \ldots, 6$, a menos de equivalência de fibrados, seus respectivos espaços totais. Aqui, o $\mathbb{S}^{2}$-fibrado trivial sobre $K b$ corresponde ao tipo $\mathcal{K}_{1}$. São três os epimorfismos de

$$
\pi_{1}(M) \cong \pi_{1}(K b)=\left\langle a_{1}, a_{2} \mid a_{1}^{2} a_{2}^{2}\right\rangle
$$

em $\mathbb{Z}_{2}$, conforme a seguinte tabela: 


\begin{tabular}{|c|c|c|}
\hline & $a_{1}$ & $a_{\mathbf{2}}$ \\
\hline $\boldsymbol{\varphi}_{\mathbf{1}}$ & $\overline{1}$ & $\overline{0}$ \\
\hline $\boldsymbol{\varphi}_{\mathbf{2}}$ & $\overline{0}$ & $\overline{1}$ \\
\hline $\boldsymbol{\varphi}_{\mathbf{3}}$ & $\overline{1}$ & $\overline{1}$ \\
\hline
\end{tabular}

Observemos que $K b_{\varphi}=T^{2}$, para $\varphi=\varphi_{3}$, e $K b_{\varphi}=K b$, para $\varphi=\varphi_{1}, \varphi_{2}$. Assim, temos:

Proposição 3.18. Sejam $\xi_{M}$ um $\mathbb{S}^{2}$-fibrado sobre $K b$ e $0 \neq[\varphi] \in H^{1}\left(M ; \mathbb{Z}_{2}\right)$. De acordo com a notação anterior,

(i) se $\xi_{M}$ é do tipo $\mathcal{K}_{1}$, ou $\mathcal{K}_{4}$, então $\xi_{M_{\varphi}}$ é do tipo

- $\mathcal{O}_{2}, \operatorname{para} \varphi=\varphi_{3}$

- $\mathcal{K}_{1}$, para $\varphi=\varphi_{1}, \varphi_{2}$;

(ii) se $\xi_{M}$ é do tipo $\mathcal{K}_{2}$, ou $\mathcal{K}_{5}$, então $\xi_{M_{\varphi}}$ é do tipo

- $\mathcal{O}_{1}$, para $\varphi=\varphi_{3}$,

- $\mathcal{K}_{1}$, para $\varphi=\varphi_{1}$,

- $\mathcal{K}_{3}$, para $\varphi=\varphi_{2}$

(iii) se $\xi_{M}$ é do tipo $\mathcal{K}_{3}$, ou $\mathcal{K}_{6}$, então $\xi_{M_{\varphi}}$ é do tipo

- $\mathcal{O}_{2}, \operatorname{para} \varphi=\varphi_{3}$

- $\mathcal{K}_{3}$, para $\varphi=\varphi_{1}, \varphi_{2}$.

Demonstração. Calculemos $s\left(w_{1}\left(\xi_{M_{\varphi}}\right)\right)$, uma vez que $w_{2}\left(\xi_{M_{\varphi}}\right)=0$.

(i) Se $\xi_{M}$ é do tipo $\mathcal{K}_{1}$, ou $\mathcal{K}_{4}$, temos $w_{1}\left(\xi_{M}\right)=0 \neq w_{1}(K b)$. Assim,

$$
w_{1}\left(\xi_{M_{\varphi}}\right)=q^{*}\left(w_{1}\left(\xi_{M}\right)\right)=0=w_{1}\left(T^{2}\right)
$$

para $\varphi=\varphi_{3}$, o que implica que $s\left(w_{1}\left(\xi_{M_{\varphi}}\right)\right)=\infty$, ou seja, $\xi_{M_{\varphi}}$ é do tipo $\mathcal{O}_{2}$. Agora,

$$
w_{1}\left(\xi_{M_{\varphi}}\right)=0 \neq w_{1}(K b),
$$

para $\varphi=\varphi_{1}, \varphi_{2}$, donde vem que $s\left(w_{1}\left(\xi_{M_{\varphi}}\right)\right)=0$, e portanto $\xi_{M_{\varphi}}$ é do tipo $\mathcal{K}_{1}$.

(ii) Se $\xi_{M}$ é do tipo $\mathcal{K}_{2}$, ou $\mathcal{K}_{5}$, temos $w_{1}\left(\xi_{M}\right) \neq 0, w_{1}\left(\xi_{M}\right) \neq w_{1}(K b)$ e $w_{1}\left(\xi_{M}\right) \smile$ $w_{1}(K b)=1$, pois $s\left(w_{1}\left(\xi_{M}\right)\right)=1$. Observemos que $w_{1}(K b)$, vista como um homomorfismo 
de $\pi_{1}(K b)$ em $\mathbb{Z}_{2}$, corresponde a $\varphi_{3}$, pois $w_{1}(K b)$ envia laços que revertem orientação em $\overline{1}$. Ainda, da estrutura multiplicativa de $H^{*}\left(K b ; \mathbb{Z}_{2}\right), w_{1}\left(\xi_{M}\right)$ vista como um epimorfismo de $\pi_{1}(K b)$ em $\mathbb{Z}_{2}$, corresponde a $\varphi_{1}$, ou $\varphi_{2}$. No entanto, segundo o critério dado por Melvin, em ambas as possibilidades, os fibrados são equivalentes. Sendo assim, sem perda de generalidade, consideremos $w_{1}\left(\xi_{M}\right)=\varphi_{1}$. Então, para $\varphi=\varphi_{3}$,

$$
w_{1}\left(\xi_{M_{\varphi}}\right)=q^{*}\left(w_{1}\left(\xi_{M}\right)\right)=\varphi_{\left.1\right|_{\operatorname{Ker}\left(\varphi_{3}\right)}} \neq 0=w_{1}\left(T^{2}\right) \text { e } w_{1}\left(\xi_{M_{\varphi}}\right) \smile w_{1}\left(T^{2}\right)=0 .
$$

Portanto, $s\left(w_{1}\left(\xi_{M_{\varphi}}\right)\right)=2$, ou seja, $\xi_{M_{\varphi}}$ é do tipo $\mathcal{O}_{1}$. Para $\varphi=\varphi_{2}$,

$$
w_{1}\left(\xi_{M_{\varphi}}\right)=q^{*}\left(w_{1}\left(\xi_{M}\right)\right)=\varphi_{\left.\right|_{\text {Ker }\left(\varphi_{2}\right)}} \neq 0 .
$$

Usando o método de Reidemeister-Schreier, temos $\operatorname{Ker}\left(\varphi_{2}\right)=\left\langle x_{1}, x_{2} \mid x_{1}^{2} x_{2}^{2}\right\rangle$, onde $x_{1}=a_{1}$ e $x_{2}=a_{2} a_{1}^{-1} a_{2}^{-1}$. Assim, $\varphi_{1}$ restrito a $\operatorname{Ker}\left(\varphi_{2}\right)$ é tal que $\varphi_{1}\left(x_{1}\right)=\overline{1}$ e $\varphi_{1}\left(x_{2}\right)=\overline{1}$, isto é, $w_{1}\left(\xi_{M_{\varphi}}\right)=w_{1}(K b)$, donde segue que $s\left(w_{1}\left(\xi_{M_{\varphi}}\right)\right)=\infty$. Portanto, $\xi_{M_{\varphi}}$ é do tipo $\mathcal{K}_{3}$. Para $\varphi=\varphi_{1}$

$$
w_{1}\left(\xi_{M_{\varphi}}\right)=q^{*}\left(w_{1}\left(\xi_{M}\right)\right)=\varphi_{\left.1\right|_{\operatorname{Ker}\left(\varphi_{1}\right)}}=0 \neq w_{1}(K b)
$$

e $\operatorname{assim} s\left(w_{1}\left(\xi_{M_{\varphi}}\right)\right)=0$, ou seja, $\xi_{M_{\varphi}}$ é do tipo $\mathcal{K}_{1}$.

(iii) Se $\xi_{M}$ é do tipo $\mathcal{K}_{3}$, ou $\mathcal{K}_{6}$, como $s\left(w_{1}\left(\xi_{M}\right)\right)=\infty$, isto é, $w_{1}\left(\xi_{M}\right)=w_{1}(K b)$, então

$$
w_{1}\left(\xi_{M_{\varphi}}\right)=q^{*}\left(w_{1}\left(\xi_{M}\right)\right)=q^{*}\left(w_{1}(K b)\right)=w_{1}\left(K b_{\varphi}\right),
$$

isto é, $s\left(w_{1}\left(\xi_{M_{\varphi}}\right)\right)=\infty$, e portanto (iii) vale.

(III) $B$ não orientável $\operatorname{com} \chi(B)<0$

Denominamos cada uma das oito classes de $\mathbb{S}^{2}$-fibrados sobre $\boldsymbol{N}_{\boldsymbol{h}}$ (superfície fechada não orientável de gênus $h \geq 3$ ) como sendo do tipo $\mathcal{N}_{i}, i=1, \ldots, 8$, conforme os invariantes dados anteriormente, organizados na seguinte tabela:

\begin{tabular}{|c|c|c|c|c|c|c|c|c|}
\hline & $\mathcal{N}_{\mathbf{1}}$ & $\mathcal{N}_{\mathbf{2}}$ & $\mathcal{N}_{\mathbf{3}}$ & $\mathcal{N}_{\mathbf{4}}$ & $\mathcal{N}_{\mathbf{5}}$ & $\mathcal{N}_{\mathbf{6}}$ & $\mathcal{N}_{\mathbf{7}}$ & $\mathcal{N}_{\mathbf{8}}$ \\
\hline $\boldsymbol{w}_{\mathbf{2}}\left(\boldsymbol{\xi}_{\boldsymbol{M}}\right)$ & 0 & 0 & 0 & 0 & 1 & 1 & 1 & 1 \\
\hline $\boldsymbol{s}\left(\boldsymbol{w}_{\mathbf{1}}\left(\boldsymbol{\xi}_{\boldsymbol{M}}\right)\right)$ & 0 & 1 & 2 & $\infty$ & 0 & 1 & 2 & $\infty$ \\
\hline
\end{tabular}

E denotamos por $E_{\mathcal{N}_{i}}$, com $i=1, \ldots, 8$, a menos de equivalência de fibrados, seus respectivos espaços totais. Aqui, o $\mathbb{S}^{2}$-fibrado trivial sobre $N_{h}(h \geq 3)$ corresponde ao tipo $\mathcal{N}_{1}$. Para cada um destes oito tipos, temos $2^{h}-1$ epimorfismos de

$$
\pi_{1}(M) \cong \pi_{1}\left(N_{h}\right)=\left\langle a_{1}, a_{2}, \ldots, a_{h} \mid a_{1}^{2} a_{2}^{2} \cdots a_{h}^{2}\right\rangle
$$

em $\mathbb{Z}_{2}$. 


\section{Observação 3.19.}

(1) Pela apresentação dada em (3.11), a classe $w_{1}\left(N_{h}\right) \in H^{1}\left(N_{h} ; \mathbb{Z}_{2}\right)$, vista como um homomorfismo de $\pi_{1}\left(N_{h}\right)$ em $\mathbb{Z}_{2}$, é tal que $w_{1}\left(N_{h}\right)\left(a_{i}\right)=\overline{1}$, para todo $i=1, \ldots, h$.

(2) Dado um homomorfismo $\varphi: \pi_{1}\left(N_{h}\right) \rightarrow \mathbb{Z}_{2}$, denotemos por $\varphi^{c}$ (o "complementar" de $\varphi$ ) o homomorfismo de $\pi_{1}\left(N_{h}\right)$ em $\mathbb{Z}_{2}$ tal que

$$
\varphi^{c}\left(a_{i}\right)=\left\{\begin{array}{lll}
\overline{0}, & \text { se } \quad \varphi\left(a_{i}\right)=\overline{1} \\
\overline{1}, & \text { se } \quad \varphi\left(a_{i}\right)=\overline{0}
\end{array}\right.
$$

$1 \leq i \leq h$.

(3) Para $\varphi=w_{1}\left(N_{h}\right)$, temos $\left(N_{h}\right)_{\varphi}=S_{h-1}(h \geq 3)$, e para $\varphi \neq w_{1}\left(N_{h}\right)$, temos $\left(N_{h}\right)_{\varphi}=$ $N_{2 h-2}$.

(4) Tomemos $x, y, z$ e $w$ geradores de um grupo $G$. Fazendo

$$
\begin{aligned}
& u:=(z w)^{-1} x(z w), \\
& v:=(z w)^{-1} y(z w),
\end{aligned}
$$

podemos substituir $x, y$ por $u, v$, e palavras do tipo $x y z w$ e $y^{2} z w$ se transformam em $z w u v$ e $z w v^{2}$, respectivamente. Agora, se consideramos

$$
\begin{gathered}
t=\left(x y x^{-1}\right)\left(y^{-1} z\right)\left(x y x^{-1}\right)^{-1}, \\
u=x y x^{-1} z^{-1} y^{-1} x^{-1}=\left(x y x^{-1}\right)\left(z^{-1} x^{-1}\right)\left(x y x^{-1}\right)^{-1}, \\
v=x y z=\left(x y x^{-1}\right) x(z x y) x^{-1}\left(x y x^{-1}\right)^{-1},
\end{gathered}
$$

podemos substituir $x, y, z$ por $t, u, v$, e uma palavra do tipo $[x, y] z^{2}$ se transforma em $t^{2} u^{2} v^{2}$

Proposição 3.20. Sejam $\xi_{M}$ um $\mathbb{S}^{2}$-fibrado sobre $N_{h}(h \geq 3)$ e $0 \neq[\varphi] \in H^{1}\left(M ; \mathbb{Z}_{2}\right)$. De acordo com a notação anterior,

(i) se $\xi_{M}$ é do tipo $\mathcal{N}_{1}$, ou $\mathcal{N}_{5}$, então $\xi_{M_{\varphi}}$ é do tipo

- $\mathcal{O}_{2}, \operatorname{para} \varphi=w_{1}\left(N_{h}\right)$,

- $\mathcal{N}_{1}$, para $\varphi \neq w_{1}\left(N_{h}\right)$;

(ii) se $\xi_{M}$ é do tipo $\mathcal{N}_{4}$, ou $\mathcal{N}_{8}$, então $\xi_{M_{\varphi}}$ é do tipo

- $\mathcal{O}_{2}, \operatorname{para} \varphi=w_{1}\left(N_{h}\right)$,

- $\mathcal{N}_{4}$, para $\varphi \neq w_{1}\left(N_{h}\right)$; 
(iii) se $\xi_{M}$ é do tipo $\mathcal{N}_{2}, \mathcal{N}_{6}, \mathcal{N}_{3}$, ou $\mathcal{N}_{7}$, então $\xi_{M_{\varphi}}$ é do tipo

- $\mathcal{O}_{1}, \operatorname{para} \varphi=w_{1}\left(N_{h}\right)$,

- $\mathcal{N}_{1}, \operatorname{para} \varphi=w_{1}\left(\xi_{M}\right)$,

- $\mathcal{N}_{4}$, para $\varphi=w_{1}\left(\xi_{M}\right)^{c}$,

- $\mathcal{N}_{3}$, para os demais epimorfismos.

Demonstração. Tal como nos resultados anteriores, $w_{2}\left(\xi_{M_{\varphi}}\right)=0$. Calculemos então, para cada tipo, o invariante $s\left(w_{1}\left(\xi_{M_{\varphi}}\right)\right)$.

(i) Se $\xi_{M}$ é do tipo $\mathcal{N}_{1}$, ou $\mathcal{N}_{5}$, então $w_{1}\left(\xi_{M}\right)=0 \neq w_{1}\left(N_{h}\right)$ o que implica em

$$
w_{1}\left(\xi_{M_{\varphi}}\right)=q^{*}\left(w_{1}\left(\xi_{M}\right)\right)=0
$$

Assim, para $\varphi=w_{1}\left(N_{h}\right)$, temos $s\left(w_{1}\left(\xi_{M_{\varphi}}\right)\right)=\infty$, pois $w_{1}\left(\left(N_{h}\right)_{\varphi}\right)=w_{1}\left(S_{h-1}\right)=0$, e para $\varphi \neq w_{1}\left(N_{h}\right)$, segue que $s\left(w_{1}\left(\xi_{M_{\varphi}}\right)\right)=0$, pois $w_{1}\left(\left(N_{h}\right)_{\varphi}\right)=w_{1}\left(N_{2 h-2}\right) \neq 0$, e isto conclui a prova de (i).

(ii) Se $\xi_{M}$ é do tipo $\mathcal{N}_{4}$, ou $\mathcal{N}_{8}$, como $s\left(w_{1}\left(\xi_{M}\right)\right)=\infty$, então $w_{1}\left(\xi_{M}\right)=w_{1}\left(N_{h}\right)$. Assim,

$$
w_{1}\left(\xi_{M_{\varphi}}\right)=q^{*}\left(w_{1}\left(\xi_{M}\right)\right)=q^{*}\left(w_{1}\left(N_{h}\right)\right)=w_{1}\left(\left(N_{h}\right)_{\varphi}\right)
$$

o que implica em $s\left(w_{1}\left(\xi_{M_{\varphi}}\right)\right)=\infty$, donde segue o resultado.

(iii) Se $\xi_{M}$ é do tipo $\mathcal{N}_{2}$, ou $\mathcal{N}_{6}$, então $w_{1}\left(\xi_{M}\right) \neq 0, w_{1}\left(\xi_{M}\right) \neq w_{1}\left(N_{h}\right)$ e $w_{1}\left(\xi_{M}\right) \smile$ $w_{1}\left(N_{h}\right)=1$, pois $s\left(w_{1}\left(\xi_{M}\right)\right)=1$. Assim, da estrutura multiplicativa de $H^{*}\left(N_{h} ; \mathbb{Z}_{2}\right)$, podemos considerar $w_{1}\left(\xi_{M}\right)=\varphi_{1}$, onde $\varphi_{1}: \pi_{1}\left(N_{h}\right) \rightarrow \mathbb{Z}_{2}$ é tal que $\varphi_{1}\left(a_{1}\right)=\overline{1}$ e $\varphi_{1}\left(a_{i}\right)=\overline{0}$, para $i=2, \ldots, h$. Daí, para $\varphi=w_{1}\left(N_{h}\right)$,

$$
w_{1}\left(\xi_{M_{\varphi}}\right)=q^{*}\left(w_{1}\left(\xi_{M}\right)\right) \neq 0=w_{1}\left(S_{h-1}\right)
$$

e $w_{1}\left(\xi_{M_{\varphi}}\right) \smile w_{1}\left(S_{h-1}\right)=0$, donde segue que $\xi_{M_{\varphi}}$ é do tipo $\mathcal{O}_{1}$. Para $\varphi=w_{1}\left(\xi_{M}\right)$, vem que

$$
w_{1}\left(\xi_{M_{\varphi}}\right)=q^{*}\left(w_{1}\left(\xi_{M}\right)\right)=0 \neq w_{1}\left(N_{2 h-2}\right),
$$

ou seja, $\xi_{M_{\varphi}}$ é do tipo $\mathcal{N}_{1}$. Para $\varphi \neq \varphi_{1}, \varphi \neq w_{1}\left(N_{h}\right)$, como $w_{1}\left(\xi_{M_{\varphi}}\right)=\varphi_{\left.1\right|_{\text {Ker }(\varphi)}} \neq 0$, a fim de compararmos $w_{1}\left(\xi_{M_{\varphi}}\right)$ com $w_{1}\left(N_{2 h-2}\right)$, e calcularmos $w_{1}\left(\xi_{M_{\varphi}}\right) \smile w_{1}\left(N_{2 h-2}\right)$, determinamos uma apresentação para $\operatorname{Ker}(\varphi)$. Assim, seja $\left\{1, a_{k}\right\}$ um sistema de Schreier, 
onde $k=\operatorname{mín}\{1, \ldots, h\}$ tal que $\varphi\left(a_{k}\right)=\overline{1}$. Usando o método de Reidemeister-Schreier, temos

$$
\operatorname{Ker}(\varphi)=\left\langle x_{1}, y_{1}, \ldots, \widehat{x}_{k}, \widehat{y}_{k}, \ldots, x_{h}, y_{h} \mid w_{k+1} \cdots w_{h} w_{1} \cdots w_{k-1} w_{k-1}^{\prime} \cdots w_{1}^{\prime} w_{h}^{\prime} \cdots w_{k+1}^{\prime}\right\rangle,
$$

onde $x_{i}=a_{i}, y_{i}=a_{k} a_{i}^{-1} a_{k}^{-1}, w_{i}=x_{i}^{2}$ e $w_{i}^{\prime}=y_{i}^{2}$, se $\varphi\left(a_{i}\right)=\overline{0}$; e $x_{i}=a_{i} a_{k}^{-1}, y_{i}=a_{k} a_{i}$, $w_{i}=x_{i} y_{i}$ e $w_{i}^{\prime}=x_{i}^{-1} y_{i}^{-1}$, se $\varphi\left(a_{i}\right)=\overline{1}$, com $i=1, \ldots, \widehat{k}, \ldots, h\left(\widehat{x}_{k}, \widehat{y}_{k}, \widehat{k}\right.$ denotam a exclusão de $x_{k}, y_{k}, k$, respectivamente). Observemos que se $k=1$, então $a_{1}$ não aparece em $x_{i}$ e aparece duas vezes em $y_{i}$, caso $\varphi\left(a_{i}\right)=\overline{0}$; e aparece uma única vez em $x_{i}$ e em $y_{i}$, caso $\varphi\left(a_{i}\right)=\overline{1}$, para algum $i=2, \ldots, h$. Se $k \neq 1$, então $a_{1}$ aparece uma única vez em $x_{1}$ e em $y_{1}$, e não aparece em $x_{i}$ e $y_{i}$, para $i=2, \ldots, h$. Realizando as transformações dadas em 3.12 e (3.13), obtemos

$$
\operatorname{Ker}(\varphi)=\left\langle u_{1}, u_{2}, \ldots, u_{2 h-1}, u_{2 h-2} \mid u_{1}^{2} u_{2}^{2} \cdots u_{2 h-1}^{2} u_{2 h-2}^{2}\right\rangle
$$

de modo que, para os epimorfismos $\varphi \neq \varphi_{1}^{c}$, existe pelo menos um par $\left(u_{j}, u_{l}\right)$ tal que $\varphi_{1}\left(u_{j}\right)=\varphi_{1}\left(u_{l}\right)=\overline{0}$, com $1 \leq j \leq 2 h-2$, e assim $w_{1}\left(\xi_{M_{\varphi}}\right) \neq w_{1}\left(N_{2 h-2}\right)$ (pois $\left.w_{1}\left(N_{2 h-2}\right)\left(u_{j}\right)=\overline{1}\right)$ e $w_{1}\left(\xi_{M_{\varphi}}\right) \smile w_{1}\left(N_{2 h-2}\right)=0$, ou seja, $\xi_{M_{\varphi}}$ é do tipo $\mathcal{N}_{3}$. Para $\varphi=\varphi_{1}^{c}$, temos $\varphi_{1}\left(u_{j}\right)=\overline{1}$, para todo $1 \leq j \leq 2 h-2$, ou seja, $w_{1}\left(\xi_{M_{\varphi}}\right)=w_{1}\left(N_{2 h-2}\right)$, e portanto $\xi_{M_{\varphi}}$ é do tipo $\mathcal{N}_{4}$. Agora, quando $\xi_{M}$ é do tipo $\mathcal{N}_{3}$, ou $\mathcal{N}_{7}$, temos $s\left(w_{1}\left(\xi_{M}\right)\right)=2$, isto é, $w_{1}\left(\xi_{M}\right) \neq 0, w_{1}\left(\xi_{M}\right) \neq w_{1}\left(N_{h}\right)$ e $w_{1}\left(\xi_{M}\right) \smile w_{1}\left(N_{h}\right)=0$. Sem perda de generalidade, podemos considerar $w_{1}\left(\xi_{M}\right)=\varphi_{h+1}$, onde $\varphi_{h+1}: \pi_{1}\left(N_{h}\right) \rightarrow \mathbb{Z}_{2}$ é tal que $\varphi_{h+1}\left(a_{1}\right)=\varphi_{h+1}\left(a_{2}\right)=\overline{1}$ e $\varphi_{h+1}\left(a_{i}\right)=\overline{0}$, para todo $i=3, \ldots, h$. Daí, procedendo de modo análogo à análise feita para os tipos $\mathcal{N}_{2}$ e $\mathcal{N}_{6}$, o resultado segue.

\subsection{2 $F=\mathbb{R} P^{2}$ e $B$ é $K(\pi, 1)$}

Seja $\xi_{M}$ um $\mathbb{R} P^{2}$-fibrado sobre $B$, onde $B$ é uma superfície fechada $K(\pi, 1)$. A menos de equivalência de fibrados, existem dois tais fibrados ([19, pág. 101]). Consideremos o subespaço $B_{1}=B \backslash \operatorname{int}\left(\mathbb{D}^{2}\right)$, sobre o qual temos o $\mathbb{R} P^{2}$-fibrado trivial. Um modelo para o espaço total do $\mathbb{R} P^{2}$-fibrado não trivial sobre $B$ é dado por

$$
E_{[(3.14)}:=\frac{\left(B_{1} \times \mathbb{R} P^{2}\right) \amalg\left(\mathbb{D}^{2} \times \mathbb{R} P^{2}\right)}{(x, y) \sim(x, \phi(x)(y))},
$$

onde $\phi(x) \in \operatorname{Homeo}\left(\mathbb{R} P^{2}\right)$ é dado pela rotação, para cada $x \in \partial B_{1}$, como no Caso 2 , da Seção 3.2. Agora, por [19, Teorema 5.16],

$$
\pi_{1}(M) \cong \pi_{1}(E) \cong \pi_{1}(B) \times\left\langle c \mid c^{2}\right\rangle
$$


donde temos o seguinte lema:

Lema 3.21. Sejam $\xi_{M}=\left(E, B, \mathbb{R} P^{2}, p\right)$, com $B$ uma superfície fechada $K(\pi, 1)$, e $0 \neq$ $[\varphi] \in H^{1}\left(M ; \mathbb{Z}_{2}\right)$. De acordo com 3.15 ,

(i) se $c \in \operatorname{Ker}(\varphi)$, então $p_{\#}(\operatorname{Ker}(\varphi)) \neq \pi_{1}(B)$;

(ii) se $c \notin \operatorname{Ker}(\varphi)$, então $p_{\#}(\operatorname{Ker}(\varphi))=\pi_{1}(B)$.

Demonstração.

(i) Suponhamos que $c \in \operatorname{Ker}(\varphi)$, ou seja, $\varphi(c)=\varphi(1, c)=\overline{0}$. Assim, para todo $w \in \pi_{1}(B)$,

$$
\varphi(w, c)=\varphi(w, 1)+\varphi(1, c)=\varphi(w, 1)
$$

Daí, como $\varphi$ é sobrejetor, existe $w^{\prime} \in \pi_{1}(B)-\{1\}$ tal que $\varphi\left(w^{\prime}, c\right) \stackrel{(3.16)}{=} \varphi\left(w^{\prime}, 1\right)=\overline{1}$. Desta forma, $w^{\prime} \notin p_{\#}(\operatorname{Ker}(\varphi))$, pois caso contrário $\varphi\left(w^{\prime}, c\right) \stackrel{(3.16)}{=} \varphi\left(w^{\prime}, 1\right)=\overline{0}$, o que é uma contradição. Portanto, temos a verificação de (i).

(ii) Como $\operatorname{Ker}(\varphi)$ é um subgrupo de $\pi_{1}(E)$, claramente $p_{\#}(\operatorname{Ker}(\varphi)) \subset \pi_{1}(B)$. Agora, se $w \in \pi_{1}(B)$, temos $p_{\#}^{-1}(w)=\{(w, 1),(w, c)\}$. Daí, se $\varphi(w, 1)=\overline{0}$, então $(w, 1) \in \operatorname{Ker}(\varphi)$, isto é, $w \in p_{\#}(\operatorname{Ker}(\varphi))$. Agora, se $\varphi(w, 1)=\overline{1}$, então $\varphi(w, c)=\varphi(1, c)+\varphi(w, 1)=$ $\overline{1}+\overline{1}=\overline{0}$, ou seja, $(w, c) \in \operatorname{Ker}(\varphi)$, o que implica que $w \in p_{\#}(\operatorname{Ker}(\varphi))$. Portanto, $p_{\#}(\operatorname{Ker}(\varphi))=\pi_{1}(B)$.

Proposição 3.22. Sejam $\xi_{M}=\left(E, B, \mathbb{R} P^{2}, p\right)$ o fibrado trivial, com $B$ uma superfície fechada $K(\pi, 1)$, e $0 \neq[\varphi] \in H^{1}\left(M ; \mathbb{Z}_{2}\right)$. Com a notação anterior, se $\varphi(c)=\overline{0}$, então $\xi_{M_{\varphi}}$ é o $\mathbb{R} P^{2}$-fibrado trivial sobre $\widehat{B}$.

Demonstração. Seja $\left(E_{\varphi}, q\right)$ o revestimento duplo de $E$ associado a $\varphi$. Como $\varphi(c)=\overline{0}$, então pelo Lema 3.21(i), $(p \circ q)_{\#}$ não é sobrejetor, e assim, pela prova do Teorema 3.5. $\xi_{M_{\varphi}}$ é um $\mathbb{R} P^{2}$-fibrado sobre $\widehat{B}$, onde $\left(\widehat{B}, q^{\prime}\right)$ é o revestimento duplo de $B$ associado ao $\operatorname{subgrupo}(p \circ q)_{\#}\left(\pi_{1}\left(E_{\varphi}\right)\right)$,

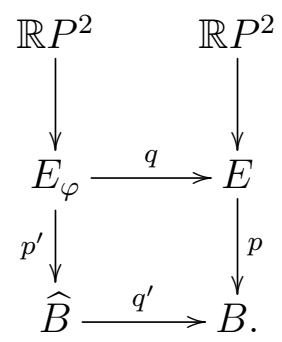

Como $\xi_{M_{\varphi}}=\left(q^{\prime}\right)^{*}\left(\xi_{M}\right)$ e $\xi_{M}$ é trivial, então $\xi_{M_{\varphi}}$ também o é.

Para os epimorfismos de $\pi_{1}(M)$ em $\mathbb{Z}_{2}$ que enviam $c$ em $\overline{1}$, obtemos os seguintes resultados: 
Proposição 3.23. Sejam $\xi_{M}=\left(E, S_{g}, \mathbb{R} P^{2}, p\right) \operatorname{com} g \geq 1$, e $0 \neq[\varphi] \in H^{1}\left(M ; \mathbb{Z}_{2}\right)$ tal que $\varphi(c)=\overline{1}$. De acordo com $(3.9)$,

(i) se $\xi_{M}$ é trivial, então $\xi_{M_{\varphi}}$ é um $\mathbb{S}^{2}$-fibrado sobre $S_{g}$ do tipo

- $\mathcal{O}_{2}$, se $\varphi\left(a_{i}\right)=\varphi\left(b_{i}\right)=\overline{0}$, para todo $i=1, \ldots, g$;

- $\mathcal{O}_{1}$, se $\varphi\left(a_{i}\right)=\overline{1}$, ou $\varphi\left(b_{i}\right)=\overline{1}$, para algum $i=1, \ldots, g$;

(ii) se $\xi_{M}$ é não trivial, então $\xi_{M_{\varphi}}$ é um $\mathbb{S}^{2}$-fibrado sobre $S_{g}$ do tipo

- $\mathcal{O}_{4}$, se $\varphi\left(a_{i}\right)=\varphi\left(b_{i}\right)=\overline{0}$, para todo $i=1, \ldots, g$;

- $\mathcal{O}_{3}$, se $\varphi\left(a_{i}\right)=\overline{1}$, ou $\varphi\left(b_{i}\right)=\overline{1}$, para algum $i=1, \ldots, g$.

Demonstração. Como $\varphi(c)=\overline{1}$, então pelo Lema 3.21(ii) e da prova do Teorema 3.5 . $\xi_{M_{\varphi}}$ é um $\mathbb{S}^{2}$-fibrado sobre $S_{g}$. Assim, se $\varphi$ é tal que $\varphi\left(a_{i}\right)=\varphi\left(b_{i}\right)=\overline{0}$, para todo $i=1, \ldots, g$, então $w_{1}\left(\xi_{M_{\varphi}}\right)=0=w_{1}\left(S_{g}\right)$, ou seja, $s\left(w_{1}\left(\xi_{M_{\varphi}}\right)\right)=\infty$. Se $\varphi$ é tal que $\varphi\left(a_{i}\right)=\overline{1}$, ou $\varphi\left(b_{i}\right)=\overline{1}$, para algum $i=1, \ldots, g$, então $w_{1}\left(\xi_{M_{\varphi}}\right) \neq 0=w_{1}\left(S_{g}\right) \mathrm{e}$ $w_{1}\left(\xi_{M_{\varphi}}\right) \smile w_{1}\left(S_{g}\right)=0$, ou seja, $s\left(w_{1}\left(\xi_{M_{\varphi}}\right)\right)=2$. Quanto a $w_{2}\left(\xi_{M_{\varphi}}\right)$, como $S_{g}=B_{1} \cup \mathbb{D}^{2}$, onde $B_{1}=S_{g} \backslash \operatorname{int}\left(\mathbb{D}^{2}\right)$, e $\xi_{M_{\varphi}}$ restrito a $\partial \mathbb{D}^{2}$ (respectivamente, restrito a $\partial B_{1}$ ) é o $\mathbb{S}^{2}$-fibrado trivial, com espaço total revestindo duplamente $\partial \mathbb{D}^{2} \times \mathbb{R} P^{2}$ (respectivamente, revestindo duplamente $\left.\partial B_{1} \times \mathbb{R} P^{2}\right)$ segue que, se $\xi_{M}$ é trivial, então $E_{\varphi}$ é obtido através da colagem de $\partial \mathbb{D}^{2} \times \mathbb{S}^{2} \operatorname{com} \partial B_{1} \times \mathbb{S}^{2}$ por $i d(x) \in \operatorname{Homeo}\left(\mathbb{S}^{2}\right)$, para cada $x \in \partial \mathbb{D}^{2}$. Se $\xi_{M}$ é não trivial, então a colagem é feita por $\phi(x) \in \operatorname{Homeo}\left(\mathbb{S}^{2}\right)$, dado pela rotação, para cada $x \in \partial \mathbb{D}^{2}$. Portanto, por [26, Lema Estrutural], $w_{2}\left(\xi_{M_{\varphi}}\right)=0$ (respectivamente, $w_{2}\left(\xi_{M_{\varphi}}\right) \neq 0$ ), se $\xi_{M}$ é trivial (respectivamente, não trivial), donde segue a prova de (i) e (ii).

Exemplo 3.24. No caso particular em que $\xi_{M}=\left(E, T^{2}, \mathbb{R} P^{2}, p\right)$ é trivial, temos

$$
\pi_{1}(M)=\left\langle a_{1}, b_{1}, c \mid\left[a_{1}, b_{1}\right], c^{2},\left[a_{1}, c\right],\left[b_{1}, c\right]\right\rangle .
$$

Assim, se $\varphi$ é um dos epimorfismos na seguinte tabela

\begin{tabular}{|l|c|c|c|}
\hline & $a_{1}$ & $b_{1}$ & $c$ \\
\hline $\boldsymbol{\varphi}_{\mathbf{1}}$ & $\overline{1}$ & $\overline{0}$ & $\overline{1}$ \\
\hline $\boldsymbol{\varphi}_{\mathbf{2}}$ & $\overline{0}$ & $\overline{1}$ & $\overline{1}$ \\
\hline $\boldsymbol{\varphi}_{\mathbf{3}}$ & $\overline{1}$ & $\overline{1}$ & $\overline{1}$ \\
\hline
\end{tabular}

determinaremos $E_{\varphi}$. Para isto, consideremos a restrição do fibrado $\xi_{M}$ a 1-célula $\mathbb{S}^{1}$, de $T^{2}$, tal que $\pi_{1}\left(\mathbb{S}^{1}\right)=\left\langle a_{1}\right\rangle$, isto é, $\xi_{\left.M\right|_{\mathbb{S}^{1}}}=\left(E^{\prime}, \mathbb{S}^{1}, \mathbb{R} P^{2}, p^{\prime}\right)$, onde $E^{\prime}=\mathbb{S}^{1} \times \mathbb{R} P^{2}$ e $p^{\prime}=p_{\left.\right|_{E^{\prime}}}$. 
Daí, seja $\psi: \pi_{1}\left(E^{\prime}\right) \rightarrow \mathbb{Z}_{2}$ o epimorfismo dado por $\psi\left(a_{1}\right)=\psi(c)=\overline{1}$ e consideremos $E_{\psi}^{\prime}$ o revestimento duplo de $E^{\prime}$ associado a $\psi$, que é o espaço total de um $\mathbb{S}^{2}$-fibrado sobre $\mathbb{S}^{1}$. Como $\operatorname{Ker}(\psi)=\left\langle a_{1} c\right\rangle$, então

$$
E_{\psi}^{\prime}=\mathbb{S}^{1} \widetilde{\times} \mathbb{S}^{2}=\frac{[0,1] \times \mathbb{S}^{2}}{(0, y) \sim(1,-y)}
$$

é o espaço total do $\mathbb{S}^{2}$-fibrado não trivial sobre $\mathbb{S}^{1}$, o qual denotaremos por $\xi$. Agora, seja $p_{1}: T^{2} \rightarrow \mathbb{S}^{1}$, a projeção na primeira coordenada. O fibrado induzido $p_{1}^{*}(\xi)$ é tal que seu espaço total é

$$
p_{1}^{*}\left(\mathbb{S}^{1} \widetilde{\times} \mathbb{S}^{2}\right)=\mathbb{S}^{1} \times\left(\mathbb{S}^{1} \widetilde{\times} \mathbb{S}^{2}\right)
$$

Consideremos-no equipado com a involução $\tau$ dada por

$$
\tau(x, \overline{(t, y)})=(x, \overline{(t,-y)})
$$

que está bem definida pois

$$
\tau(x, \overline{(0, y)})=(x, \overline{(0,-y)})=(x, \overline{(1, y)})=\tau(x, \overline{(1,-y)})
$$

é livre e é tal que $\left(\mathbb{S}^{1} \times\left(\mathbb{S}^{1} \widetilde{\times} \mathbb{S}^{2}\right)\right) / \tau=T^{2} \times \mathbb{R} P^{2}$, ou seja, $\left(\mathbb{S}^{1} \times\left(\mathbb{S}^{1} \widetilde{\times} \mathbb{S}^{2}\right), q\right)$ é um revestimento duplo de $T^{2} \times \mathbb{R} P^{2}$, e o mesmo corresponde ao epimorfismo $\varphi_{1}$. Logo, pela proposição anterior, para $\varphi_{2}$ e $\varphi_{3}$, temos que $M_{\varphi} \simeq \mathbb{S}^{1} \times\left(\mathbb{S}^{1} \widetilde{\times} \mathbb{S}^{2}\right)$.

Para $B=K b$, temos

$$
\pi_{1}(M)=\left\langle a_{1}, a_{2}, c \mid a_{1}^{2} a_{2}^{2}, c^{2},\left[a_{1}, c\right],\left[a_{2}, c\right]\right\rangle
$$

Assim, os epimorfismos de $\pi_{1}(M)$ em $\mathbb{Z}_{2}$ que enviam $c$ em $\overline{1}$ são dados na seguinte tabela:

\begin{tabular}{|c|c|c|c|}
\hline & $a_{\mathbf{1}}$ & $\boldsymbol{a}_{\mathbf{2}}$ & $\boldsymbol{c}$ \\
\hline $\boldsymbol{\varphi}_{\mathbf{1}}$ & $\overline{0}$ & $\overline{0}$ & $\overline{1}$ \\
\hline $\boldsymbol{\varphi}_{\mathbf{2}}$ & $\overline{1}$ & $\overline{0}$ & $\overline{1}$ \\
\hline $\boldsymbol{\varphi}_{\mathbf{3}}$ & $\overline{0}$ & $\overline{1}$ & $\overline{1}$ \\
\hline $\boldsymbol{\varphi}_{\mathbf{4}}$ & $\overline{1}$ & $\overline{1}$ & $\overline{1}$ \\
\hline
\end{tabular}

Proposição 3.25. Sejam $\xi_{M}=\left(E, K b, \mathbb{R} P^{2}, p\right)$ e $0 \neq[\varphi] \in H^{1}\left(M ; \mathbb{Z}_{2}\right)$ tal que $\varphi(c)=\overline{1}$. Com a notação da tabela anterior,

(i) se $\xi_{M}$ é trivial, então $\xi_{M_{\varphi}}$ é um $\mathbb{S}^{2}$-fibrado sobre $K b$ do tipo 
- $\mathcal{K}_{1}$, se $\varphi=\varphi_{1}$,

- $\mathcal{K}_{2}$, se $\varphi=\varphi_{2}, \varphi_{3}$,

- $\mathcal{K}_{3}$, se $\varphi=\varphi_{4}$

(ii) se $\xi_{M}$ é não trivial, então $\xi_{M_{\varphi}}$ é um $\mathbb{S}^{2}$-fibrado sobre $S_{g}$ do tipo

- $\mathcal{K}_{4}$, se $\varphi=\varphi_{1}$,

- $\mathcal{K}_{5}$, se $\varphi=\varphi_{2}, \varphi_{3}$,

- $\mathcal{K}_{6}$, se $\varphi=\varphi_{4}$.

Demonstração. Pelo Lema 3.21(ii), $\xi_{M_{\varphi}}$ é um $\mathbb{S}^{2}$-fibrado sobre $K b$. Daí, para concluírmos sobre o tipo de $\xi_{M_{\varphi}}$, basta calcularmos $s\left(w_{1}\left(\xi_{M_{\varphi}}\right)\right)$, já que $w_{2}\left(\xi_{M_{\varphi}}\right)$ segue como na prova da proposição anterior. Se $\varphi=\varphi_{1}$, então $w_{1}\left(\xi_{M_{\varphi}}\right)=0 \neq w_{1}(K b)$, isto é, $s\left(w_{1}\left(\xi_{M_{\varphi}}\right)\right)=0$. Se $\varphi=\varphi_{2}$, ou $\varphi=\varphi_{3}$, então $w_{1}\left(\xi_{M_{\varphi}}\right) \neq 0, w_{1}\left(\xi_{M_{\varphi}}\right) \neq w_{1}(K b)$ e $w_{1}\left(\xi_{M_{\varphi}}\right) \smile w_{1}(K b)=1$, isto é, $s\left(w_{1}\left(\xi_{M_{\varphi}}\right)\right)=1$. E finalmente, se $\varphi=\varphi_{4}$, então $w_{1}\left(\xi_{M_{\varphi}}\right)=w_{1}(K b)$, isto é, $s\left(w_{1}\left(\xi_{M_{\varphi}}\right)\right)=\infty$.

No caso em que $B=N_{h}(h \geq 3)$, temos

$$
\pi_{1}(M)=\left\langle a_{1}, \ldots, a_{h} \mid a_{1}^{2} \cdots a_{h}^{2}, c^{2},\left[a_{i}, c\right], i=1, \ldots, h\right\rangle
$$

Proposição 3.26. Sejam $\xi_{M}=\left(E, N_{h}, \mathbb{R} P^{2}, p\right)$, com $h \geq 3$, e $0 \neq[\varphi] \in H^{1}\left(M ; \mathbb{Z}_{2}\right)$ tal que $\varphi(c)=\overline{1}$. Logo, de acordo com a notação anterior,

(i) se $\xi_{M}$ é trivial, então $\xi_{M_{\varphi}}$ é um $\mathbb{S}^{2}$-fibrado sobre $N_{h}$ do tipo

- $\mathcal{N}_{1}$, se $\varphi\left(a_{i}\right)=\overline{0}$, para todo $i=1, \ldots, h$,

- $\mathcal{N}_{2}$, se $\varphi\left(a_{i}\right)=\overline{1}$, para uma quantidade ímpar de $i$ 's, e $\varphi\left(a_{i}\right)=\overline{0}$, para algum $i=1, \ldots, h$,

- $\mathcal{N}_{3}$, se $\varphi\left(a_{i}\right)=\overline{1}$, para uma quantidade par de $i$ 's, e $\varphi\left(a_{i}\right)=\overline{0}$, para algum $i=$ $1, \ldots, h$,

- $\mathcal{N}_{4}$, se $\varphi\left(a_{i}\right)=\overline{1}$, para todo $i=1, \ldots, h$;

(ii) se $\xi_{M}$ é não trivial, então $\xi_{M_{\varphi}}$ é um $\mathbb{S}^{2}$-fibrado sobre $S_{g}$ do tipo

- $\mathcal{N}_{5}$, se $\varphi\left(a_{i}\right)=\overline{0}$, para todo $i=1, \ldots, h$, 
- $\mathcal{N}_{6}$, se $\varphi\left(a_{i}\right)=\overline{1}$, para uma quantidade ímpar de $i$ 's, e $\varphi\left(a_{i}\right)=\overline{0}$, para algum $i=1, \ldots, h$,

- $\mathcal{N}_{7}$, se $\varphi\left(a_{i}\right)=\overline{1}$, para uma quantidade par de $i$ 's, e $\varphi\left(a_{i}\right)=\overline{0}$, para algum $i=$ $1, \ldots, h$,

- $\mathcal{N}_{8}$, se $\varphi\left(a_{i}\right)=\overline{1}$, para todo $i=1, \ldots, h$.

Demonstração. O resultado segue de modo análogo às provas das proposições anteriores.

Observação 3.27. Nos resultados anteriores, para o caso em que $\xi_{M}$ é trivial e $\varphi(x)=\overline{0}$, para todo gerador $x$ de $\pi_{1}(B)$ ( $B$ sendo uma superfície fechada $K(\pi, 1)$ ), $\xi_{M_{\varphi}}$ também é trivial, ou seja, $M_{\varphi} \simeq E_{\varphi}=B \times \mathbb{S}^{2}$, e $E_{\varphi}$ está equipado com a involução livre da forma diagonal $i d \times A\left(A\right.$ é a involução antipodal sobre $\left.\mathbb{S}^{2}\right)$.

\subsection{3 $F$ é $K(\pi, 1)$ e $B=\mathbb{S}^{2}$}

Seja $\xi_{M}$ um $F$-fibrado sobre $\mathbb{S}^{2}$, com $F$ uma superfície fechada $K(\pi, 1)$. Para $F \neq T^{2}, K b$, existe apenas uma classe de tal fibrado, pois

$$
\left[\mathbb{S}^{2}, B E(F)\right]=\left[\mathbb{S}^{2}, K\left(\operatorname{Out}\left(\pi_{1}(F)\right), 1\right)\right]=\operatorname{Hom}\left(\pi_{1}\left(\mathbb{S}^{2}\right), \operatorname{Out}\left(\pi_{1}(F)\right)\right) / \sim=1
$$

([19, Teorema 5.1]), e

$$
\pi_{1}(M) \cong \pi_{1}(E) \cong \pi_{1}(F)
$$

donde temos o seguinte resultado:

Proposição 3.28. Sejam $\xi_{M}=\left(E, \mathbb{S}^{2}, F, p\right)$, com $F$ uma superfície fechada $K(\pi, 1)$ diferente de $T^{2}$ e $K b$, e $0 \neq[\varphi] \in H^{1}\left(M ; \mathbb{Z}_{2}\right)$. Então, $\xi_{M_{\varphi}}$ é o $F_{\varphi}$-fibrado sobre $\mathbb{S}^{2}$, único a menos de equivalência de fibrados.

Demonstração. Segue da prova do Teorema 3.5 e de [19, Teorema 5.1], usando o fato de que $F_{\varphi}$ é $K(\pi, 1)$ e $\chi\left(F_{\varphi}\right)<0$.

\section{Observação 3.29.}

(1) No caso em que $F=T^{2}$, um $T^{2}$-fibrado sobre $\mathbb{S}^{2}$ é determinado pela classe de homotopia da aplicação de colagem que é um elemento de $\pi_{1}\left(\operatorname{Homeo}\left(T^{2}\right)\right)$. Como as componentes conexas de Homeo $\left(T^{2}\right)$ têm o mesmo tipo de homotopia, se denotamos por $C_{i d}$ a 
componente da identidade, então por [9],

$$
\pi_{1}\left(\operatorname{Homeo}\left(T^{2}\right)\right) \cong \pi_{1}\left(C_{i d}\right) \cong \pi_{1}\left(T^{2}\right)
$$

ou seja, os $T^{2}$-fibrados sobre $\mathbb{S}^{2}$ são classificados pelas classes de laços em $T^{2}$.

(2) Agora, um $K b$-fibrado sobre $\mathbb{S}^{2}$ é determinado pela classe de homotopia da aplicação de colagem, que é um elemento de $\pi_{1}(\operatorname{Homeo}(K b))$. Daí, por [9],

$$
\pi_{1}(\operatorname{Homeo}(K b))=\pi_{1}\left(C_{i d}\right) \cong \pi_{1}(S O(2))=\mathbb{Z}
$$

(3) Não faremos o reconhecimento dos revestimentos duplos para tais fibrados.

\subsection{4 $F$ é $K(\pi, 1)$ e $B=\mathbb{R} P^{2}$}

Seja $\xi_{M}=\left(E, \mathbb{R} P^{2}, F, p\right)$, com $F$ uma superfície fechada $K(\pi, 1)$. Da sequência (3.1), temos

$$
\cdots \longrightarrow \pi_{2}\left(\mathbb{R} P^{2}\right) \stackrel{\partial}{\longrightarrow} \pi_{1}(F) \longrightarrow \pi_{1}(M) \longrightarrow \mathbb{Z}_{2} \longrightarrow 1,
$$

onde $\partial$ é o homomorfismo conectante.

Proposição 3.30. De acordo com a notação anterior, sejam $\xi_{M}=\left(E, \mathbb{R} P^{2}, F, p\right)$, com $F$ uma superfície fechada $K(\pi, 1)$ tal que $\chi(F)<0$, ou $F=T^{2}, K b$ e $\partial=1$, e $0 \neq[\varphi] \in$ $H^{1}\left(M ; \mathbb{Z}_{2}\right)$. Assim,

(i) se $\varphi_{\left.\right|_{\pi_{1}(F)}}$ é trivial, então $\xi_{M_{\varphi}}$ é um $F$-fibrado sobre $\mathbb{S}^{2}$;

(ii) se $\varphi_{\left.\right|_{\pi_{1}(F)}}$ não é trivial, então $\xi_{M_{\varphi}}$ é um $\widehat{F}$-fibrado sobre $\mathbb{R} P^{2}$.

Demonstração. Das hipóteses, temos a sequência exata curta

$$
1 \longrightarrow \pi_{1}(F) \longrightarrow \pi_{1}(E) \stackrel{p_{\#}}{\longrightarrow} \mathbb{Z}_{2} \longrightarrow 1
$$

Assim, se $\varphi$ restrito a $\pi_{1}(F)$ é trivial, então $\pi_{1}(F) \subset \pi_{1}\left(E_{\varphi}\right)$. Logo, como $\pi_{1}\left(E_{\varphi}\right)$ e $\pi_{1}(F)$ são subgrupos de índice dois em $\pi_{1}(E)$, segue que $\pi_{1}\left(E_{\varphi}\right)=\pi_{1}(F)$. Daí, $p_{\#}\left(\pi_{1}\left(E_{\varphi}\right)\right)=1$, ou seja, $p_{\#}\left(\pi_{1}\left(E_{\varphi}\right)\right)$ é um subgrupo de índice dois em $\pi_{1}\left(\mathbb{R} P^{2}\right)$. Agora, se a restrição de $\varphi$ a $\pi_{1}(F)$ não é trivial, então existe $y \in \pi_{1}\left(E_{\varphi}\right)$ tal que $y \notin \pi_{1}(F)$. De fato, suponhamos que $\pi_{1}\left(E_{\varphi}\right) \subset \pi_{1}(F)$. Daí, $\pi_{1}\left(E_{\varphi}\right)=\pi_{1}(F)$, o que implica que $\varphi\left(\pi_{1}(F)\right)=\{\overline{0}\}$, o que é uma contradição. Então, $p_{\#}(y)=\overline{1}$, ou seja, $p_{\#}\left(\pi_{1}\left(E_{\varphi}\right)\right)=\pi_{1}\left(\mathbb{R} P^{2}\right)$. Portanto, (i) e (ii) seguem da prova do Teorema 3.5 . 


\section{Observação 3.31 .}

(1) Segundo [19, Lema 5.21], se $\chi(F)<0$, ou $\chi(F)=0$ e $\partial=1$, um $F$-fibrado sobre $\mathbb{R} P^{2}$ é determinado, a menos de equivalência de fibrados, pela extensão correspondente dos grupos fundamentais.

(2) Pela proposição anterior (item (i)), para $F \neq T^{2}, K b$, temos $M_{\varphi} \simeq F \times \mathbb{S}^{2}$.

(3) Não faremos o reconhecimento das 4 -variedades $M$ tais que $\xi_{M}=\left(E, \mathbb{R} P^{2}, F, p\right)$, quando $\chi(F)=0$ e $\partial \neq 1$.

\subsection{Considerações sobre a subfamília $\mathcal{F}_{3}$}

Segundo [19, Teorema 5.2], uma 4-variedade fechada $M$ é um elemento da subfamília $\mathcal{F}_{3}$, ou seja, $\xi_{M}$ é um $F$-fibrado sobre $B$, com $B$ e $F$ superfícies fechadas $K(\pi, 1)$, se, e somente se, $\chi(M)=\chi(B) \chi(F)$ e $\pi_{1}(M)$ é uma extensão de $\pi_{1}(B)$ por $\pi_{1}(F)$, e além disso, toda extensão de $\pi_{1}(B)$ por $\pi_{1}(F)$ é realizada por algum fibrado de superfície, que é determinado, a menos de equivalência de fibrados, pela extensão. No entanto, esta caracterização algébrica não foi suficiente para fazermos a descrição dos elementos desta subfamília, e nos parece um tanto abstrato a identificação dos seus revestimentos duplos. Portanto, não tentaremos fazer esta análise. 


\section{Capítulo 4}

\section{PBU para revestimentos duplos de fibrados de superfície}

Como vimos no capítulo anterior, não foi possível detectarmos todas as involuções livres sobre os elementos da família $\mathcal{F}$. Sendo assim, neste capítulo, apresentaremos alguns resultados mais gerais à respeito da propriedade de Borsuk-Ulam para os revestimentos duplos de elementos de $\mathcal{F}$, bem como mais específicos para os revestimentos duplos de elementos das subfamílias $\mathcal{F}_{1}$ e $\mathcal{F}_{2}$. Recordemos que, dados $M \in \mathcal{F}$ e $[\varphi]$ uma classe não nula de $H^{1}\left(M ; \mathbb{Z}_{2}\right)$, denotamos por $M_{\varphi}$ o revestimento duplo de $M$ associado ao epimorfismo $\varphi: \pi_{1}(M) \rightarrow \mathbb{Z}_{2}$, e por $\tau_{\varphi}$ a involução livre sobre $M_{\varphi}$ dada pela "deck transformation". Como $E \stackrel{f}{\simeq} M$, pelo Teorema 3.5 . $M_{\varphi}$ é um elemento de $\mathcal{F}$ e $M_{\varphi} \simeq E_{\varphi}$, onde $\left(E_{\varphi}, q\right)$ é o revestimento duplo $E$ associado a $\varphi^{\prime}:=\varphi \circ f_{\#}$, equipado com a involução livre $\tau_{\varphi}^{\prime}$ (dada pela "deck transformation"). Uma vez que $\operatorname{dim}\left(M_{\varphi}\right)=4$, os casos a serem analisados são $n=2,3,4$, para triplas do tipo $\left(M_{\varphi}, \tau_{\varphi} ; \mathbb{R}^{n}\right)$.

\subsection{Resultados gerais}

De modo análogo ao Corolário 1.17, prova-se:

Corolário 4.1. Dados pares $\left(X, \tau_{1}\right)$ e $\left(Y, \tau_{2}\right)$, com $X$ e $Y$ espaços quaisquer, sejam $w_{X} \in$ $H^{1}\left(X / \tau_{1} ; \mathbb{Z}_{2}\right)$ e $w_{Y} \in H^{1}\left(Y / \tau_{2} ; \mathbb{Z}_{2}\right)$ as respectivas classes características dos $\mathbb{Z}_{2}$-fibrados principais $X \rightarrow X / \tau_{1}$ e $Y \rightarrow Y / \tau_{2}$. Se existe equivalência de homotopia $f: X / \tau_{1} \rightarrow Y / \tau_{2}$ tal que $f^{*}\left(w_{Y}\right)=w_{X}$, então a propriedade de Borsuk-Ulam vale para $\left(X, \tau_{1} ; \mathbb{R}^{n}\right)$ se, e somente se, ela vale para $\left(Y, \tau_{2} ; \mathbb{R}^{n}\right)$. 
De acordo com a notação anterior, temos:

Lema 4.2. A propriedade de Borsuk-Ulam vale para $\left(M_{\varphi}, \tau_{\varphi} ; \mathbb{R}^{n}\right)$ se, e somente se, ela vale para $\left(E_{\varphi}, \tau_{\varphi}^{\prime} ; \mathbb{R}^{n}\right)$.

Demonstração. Desde que a equivalência de homotopia $f: E \rightarrow M$ é tal que $f^{*}([\varphi])=$ $\left[\varphi^{\prime}\right]$, o resultado segue do Corolário anterior.

Mais geralmente, para os revestimentos duplos de elementos de $\mathcal{F}$, temos o seguinte resultado:

Teorema 4.3. Sejam $M \in \mathcal{F}$, $\operatorname{com} \xi_{M}=(E, B, F, p)$ um fibrado de superfície correspondente, e $0 \neq[\varphi] \in H^{1}\left(M ; \mathbb{Z}_{2}\right)$. De acordo com a notação anterior, se $(p \circ q)_{\#}$ não é sobrejetor, então a propriedade de Borsuk-Ulam não vale para $\left(M_{\varphi}, \tau_{\varphi} ; \mathbb{R}^{n}\right)$ quando $n=3,4$.

Demonstração. De acordo com nossas hipóteses, da prova do Teorema 3.5.

$$
\xi_{M_{\varphi}}=\left(q^{\prime}\right)^{*}\left(\xi_{M}\right)=\left(E_{\varphi}, \widehat{B}, F, p_{2}\right)
$$

onde $E_{\varphi}=\left\{(u, v) \in E \times \widehat{B} \mid p(u)=q^{\prime}(v)\right\}$ e $\left(\widehat{B}, q^{\prime}\right)$ é o revestimento duplo de $B$ associado ao subgrupo de índice dois $(p \circ q)_{\#}\left(\pi_{1}\left(E_{\varphi}\right)\right)$ de $\pi_{1}(B)$,

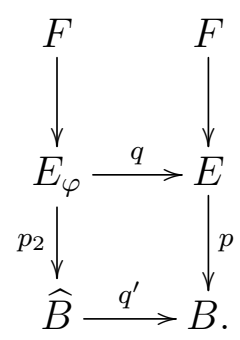

Denotemos por $\widehat{\beta}$ a involução livre sobre $\widehat{B}$ dada pela "deck transformation".

Afirmação. A projeção $p_{2}:\left(E_{\varphi}, \tau_{\varphi}^{\prime}\right) \rightarrow(\widehat{B}, \widehat{\beta})$ é equivariante.

Com efeito, seja $(u, v) \in E_{\varphi}$. Como $q$ é uma aplicação de revestimento duplo

$$
q(u, v)=q\left(\tau_{\varphi}^{\prime}(u, v)\right)=u
$$

o que implica em $\tau_{\varphi}^{\prime}(u, v)=\left(u, v^{\prime}\right)$, com $v^{\prime} \neq v$. Daí, da comutatividade do diagrama acima, segue que $v^{\prime}=\widehat{\beta}(v)$. Portanto,

$$
p_{2}\left(\tau_{\varphi}^{\prime}(u, v)\right)=p_{2}\left(u, v^{\prime}\right)=v^{\prime}=\widehat{\beta}(v)=\widehat{\beta}\left(p_{2}(u, v)\right)
$$


donde segue a equivariância de $p_{2}$.

Desta forma, como a propriedade de Borsuk-Ulam não vale para $\left(\widehat{B}, \widehat{\beta} ; \mathbb{R}^{n}\right)$ quando $n=$ 3, 4 (Lema 1.18(3) e (1)), pela Proposição 1.16 (equivalência (i) e (ii)), existem aplicações equivariantes de $\widehat{B}$ nas esferas $\mathbb{S}^{2}$ e $\mathbb{S}^{3}$. Assim, compondo-as com a projeção equivariante $p_{2}$, obtemos aplicações equivariantes de $E_{\varphi}$ em $\mathbb{S}^{2}$ e $\mathbb{S}^{3}$ (lembrando que composta de equivariantes também é equivariante). Logo, pela Proposição 1.16 (equivalência (i) e (ii)), segue que a propriedade de Borsuk-Ulam não vale para $\left(E_{\varphi}, \tau_{\varphi}^{\prime} ; \mathbb{R}^{n}\right)$ quando $n=3,4$. Portanto, o resultado segue do lema anterior.

Nas próximas seções analisaremos a propriedade de Borsuk-Ulam para os revestimentos duplos de elementos das subfamílias $\mathcal{F}_{1}$ e $\mathcal{F}_{2}$. A menos que se diga o contrário, adotaremos as seguintes apresentações

$$
\begin{gathered}
\pi_{1}\left(S_{g}\right)=\left\langle a_{1}, b_{1}, \ldots, a_{g}, b_{g} \mid\left[a_{1}, b_{1}\right] \cdots\left[a_{g}, b_{g}\right]\right\rangle(g \geq 1), \\
\pi_{1}\left(N_{h}\right)=\left\langle a_{1} \ldots, a_{h} \mid a_{1}^{2} \cdots a_{h}^{2}\right\rangle(h \geq 1) .
\end{gathered}
$$

\subsection{PBU para revestimentos duplos de elementos de $\mathcal{F}_{1}$}

Para elementos da subfamília $\mathcal{F}_{1}$ que admitem involuções livres, temos o seguinte resultado:

Proposição 4.4. Seja $(M, \tau)$ um par tal que $M \in \mathcal{F}_{1}$. Então, a propriedade de BorsukUlam vale para $\left(M, \tau ; \mathbb{R}^{2}\right)$.

Demonstração. Como $\pi_{1}(M)$ é finito, o resultado segue do Corolário 1.21 .

Observação 4.5. Em particular, sempre temos a validade da propriedade de BorsukUlam para $\left(M_{\varphi}, \tau_{\varphi} ; \mathbb{R}^{2}\right)$, para todo elemento $M$ de $\mathcal{F}_{1}$ que admite revestimento duplo.

Assim, falta verificarmos a propriedade de Borsuk-Ulam para $\left(M_{\varphi}, \tau_{\varphi} ; \mathbb{R}^{n}\right)$ quando $n=3,4$. Como consequência dos Teoremas 3.5 e 4.3 , temos:

Corolário 4.6. Se $\xi_{M}=\left(E, \mathbb{R} P^{2}, \mathbb{S}^{2}, p\right)$ e $0 \neq[\varphi] \in H^{1}\left(M ; \mathbb{Z}_{2}\right)$, então a propriedade de Borsuk-Ulam não vale para $\left(M_{\varphi}, \tau_{\varphi} ; \mathbb{R}^{n}\right)$ quando $n=3,4$. 


\section{Observação 4.7 .}

(1) Para $\xi_{M}=\left(E, \mathbb{S}^{2}, \mathbb{S}^{2}, p\right)$ não faz sentido estudar a propriedade de Borsuk-Ulam para $\left(M_{\varphi}, \tau_{\varphi} ; \mathbb{R}^{n}\right)$, pois $M$ é simplesmente conexa.

(2) Se $\xi_{M}=\left(E, \mathbb{S}^{2}, \mathbb{R} P^{2}, p\right)$ é trivial e $0 \neq[\varphi] \in H^{1}\left(M ; \mathbb{Z}_{2}\right)$, então a propriedade de Borsuk-Ulam não vale para a tripla $\left(M_{\varphi}, \tau_{\varphi} ; \mathbb{R}^{n}\right)$ quando $n=3,4$. De fato, da prova da Proposição 3.9, $M_{\varphi} \simeq \mathbb{S}^{2} \times \mathbb{S}^{2}$ e a involução livre $\tau_{\varphi}^{\prime}$ sobre $\mathbb{S}^{2} \times \mathbb{S}^{2}$ é da forma diagonal dada por $\tau_{\varphi}^{\prime}(x, y)=(x,-y)$. Portanto, pela Proposição 2.9, a propriedade de Borsuk-Ulam não vale para $\left(\mathbb{S}^{2} \times \mathbb{S}^{2}, \tau_{\varphi}^{\prime} ; \mathbb{R}^{n}\right)$ quando $n=3$, 4, e daí a observação segue do Lema 4.2 .

(3) Se $\xi_{M}=\left(E, \mathbb{R} P^{2}, \mathbb{R} P^{2}, p\right)$ é trivial, então existem três revestimentos duplos de $M$, associados aos epimorfismos apresentados na Tabela 3.1. Da prova da Proposição 3.11. $M_{\varphi} \simeq \mathbb{S}^{2} \times \mathbb{R} P^{2}$ e $\tau_{\varphi}^{\prime}$ é da forma diagonal, para $\varphi=\varphi_{1}, \varphi_{2}$. Portanto, a propriedade de Borsuk-Ulam não vale para $\left(M_{\varphi}, \tau_{\varphi} ; \mathbb{R}^{n}\right)$ quando $n=3,4$ e $\varphi=\varphi_{1}, \varphi_{2}$.

(4) Ainda não podemos dizer sobre a validade da propriedade de Borsuk-Ulam para as ternas $\left(M_{\varphi}, \tau_{\varphi} ; \mathbb{R}^{n}\right)$ quando $n=3,4$, nos seguintes casos:

- $\xi_{M}$ é o $\mathbb{R} P^{2}$-fibrado não trivial sobre $\mathbb{S}^{2}$;

- $\xi_{M}$ é o $\mathbb{R} P^{2}$-fibrado trivial sobre $\mathbb{R} P^{2}$ e $\varphi=\varphi_{3}$ (Tabela 3.1);

- $\xi_{M}$ é o $\mathbb{R} P^{2}$-fibrado não trivial sobre $\mathbb{R} P^{2}$.

\subsection{PBU para revestimentos duplos de elementos de $\mathcal{F}_{2}$}

Dividiremos a subfamília $\mathcal{F}_{2}$ em quatro casos conforme subseções a seguir.

\subsection{1 $F=\mathbb{S}^{2}$ e $B$ é $K(\pi, 1)$}

Seja $\xi_{M}=\left(E, B, \mathbb{S}^{2}, p\right)$, com $B$ uma superfície fechada $K(\pi, 1)$. Daí, $\pi_{1}(M) \cong \pi_{1}(B)$, e assim obtemos:

Proposição 4.8. Sejam $\xi_{M}=\left(E, B, \mathbb{S}^{2}, p\right)$, com $B$ uma superfície fechada $K(\pi, 1)$, e $0 \neq[\varphi] \in H^{1}\left(M ; \mathbb{Z}_{2}\right)$. A propriedade de Borsuk-Ulam vale para $\left(M_{\varphi}, \tau_{\varphi} ; \mathbb{R}^{2}\right)$ se, e somente se, $B$ é não orientável e $\varphi$ envia uma quantidade ímpar de geradores de $\pi_{1}(B)$ em $\overline{1}$.

Demonstração. Para a prova, estudaremos a fatoração do diagrama (*), do Teorema 1.20 . Se $B$ é orientável, podemos definir homomorfismo $\eta$ de $\pi_{1}(M) \cong \pi_{1}(B)$ em $\mathbb{Z}$ tal que 
$i_{\#} \circ \eta=\varphi$, para cada epimorfismo $\varphi$ de $\pi_{1}(M)$ em $\mathbb{Z}_{2}$. Com efeito, pela apresentação dada em (4.1), basta definirmos $\eta$ em $a_{i}$ e $b_{i}$ como sendo um inteiro par ou ímpar, caso $\varphi\left(a_{i}\right)$ e $\varphi\left(b_{i}\right)$ sejam $\overline{0}$ ou $\overline{1}(i=1, \ldots, g)$. De 4.2 , o mesmo acontece quando $B=N_{h}$ e $\varphi\left(a_{j}\right)=\overline{1}$ para uma quantidade par de $j$ 's $(j=1, \ldots, h)$, e isto prova a implicação “”". Reciprocamente, seja $B=N_{h}$ e $\varphi: \pi_{1}\left(N_{h}\right) \rightarrow \mathbb{Z}_{2}$ tal que $\varphi\left(a_{j}\right)=\overline{1}$ para uma quantidade ímpar de $j$ 's $(j=1, \ldots, h)$. Sem perda de generalidade consideremos que $\varphi\left(a_{1}\right)=\overline{1}$. Suponhamos que a propriedade de Borsuk-Ulam não valha para $\left(M_{\varphi}, \tau_{\varphi} ; \mathbb{R}^{2}\right)$. Assim, existe homomorfismo $\eta: \pi_{1}(M) \rightarrow \mathbb{Z}$ tal que $i_{\#} \circ \eta=\varphi$, ou seja, $\eta\left(a_{1}\right)$ deve ser um inteiro ímpar. No entanto, da relação $a_{1}^{2} \cdots a_{h}^{2}=1$, temos

$$
\eta\left(a_{1}\right)=-\left(\eta\left(a_{2}\right)+\cdots+\eta\left(a_{h}\right)\right)
$$

onde o segundo membro desta igualdade é um inteiro par, o que é uma contradição.

Corolário 4.9. Sejam $\xi_{M}=\left(E, B, \mathbb{S}^{2}, p\right)$, com $B$ uma superfície fechada $K(\pi, 1)$, e $0 \neq[\varphi] \in H^{1}\left(M ; \mathbb{Z}_{2}\right)$. Então, a propriedade de Borsuk-Ulam não vale para $\left(M_{\varphi}, \tau_{\varphi} ; \mathbb{R}^{n}\right)$ quando $n=3,4$.

Demonstração. Segue do Teorema 4.3 .

\subsection{2 $F=\mathbb{R} P^{2}$ e $B$ é $K(\pi, 1)$}

Seja $\xi_{M}=\left(E, B, \mathbb{R} P^{2}, p\right)$, com $B$ uma superfície fechada $K(\pi, 1)$. Lembremos que

$$
\pi_{1}(M) \cong \pi_{1}(B) \times \mathbb{Z}_{2}=\pi_{1}(B) \times\left\langle c \mid c^{2}\right\rangle
$$

(como em (3.15)). Assim, obtemos:

Proposição 4.10. Sejam $\xi_{M}=\left(E, B, \mathbb{R} P^{2}, p\right)$, com $B$ uma superfície fechada $K(\pi, 1)$, e $0 \neq[\varphi] \in H^{1}\left(M ; \mathbb{Z}_{2}\right)$. De acordo com a notação anterior,

(i) se $\varphi(c)=\overline{0}$, então a propriedade de Borsuk-Ulam vale para $\left(M_{\varphi}, \tau_{\varphi} ; \mathbb{R}^{2}\right)$ se, e somente se, $B$ é não orientável e $\varphi$ envia uma quantidade ímpar de geradores de $\pi_{1}(B)$ em $\overline{1}$;

(ii) se $\varphi(c)=\overline{1}$, então a propriedade de Borsuk-Ulam vale para $\left(M_{\varphi}, \tau_{\varphi} ; \mathbb{R}^{2}\right)$.

Demonstração. Através da análise da fatoração do diagrama (*), do Teorema 1.20, usando as apresentações 4.1) e 4.2), as condições do item (i) são necessárias e suficientes. Para a prova de (ii), suponhamos que a propriedade de Borsuk-Ulam não valha para $\left(M_{\varphi}, \tau_{\varphi} ; \mathbb{R}^{2}\right)$. Então, existe homomorfismo $\eta: \pi_{1}(M) \rightarrow \mathbb{Z}$ tal que o diagrama $(*)$ se fatora. Logo, $\eta(c)$ 
deve ser um inteiro ímpar, mas da relação $c^{2}=1$, segue que $\eta(c)=0$, o que é uma contradição.

Como consequência do Lema 3.21(i) e do Teorema 4.3, temos:

Corolário 4.11. Sejam $\xi_{M}=\left(E, B, \mathbb{R} P^{2}, p\right)$, com $B$ uma superfície fechada $K(\pi, 1)$, e $0 \neq[\varphi] \in H^{1}\left(M ; \mathbb{Z}_{2}\right)$. Se $\varphi(c)=\overline{0}$, então a propriedade de Borsuk-Ulam não vale para $\left(M_{\varphi}, \tau_{\varphi} ; \mathbb{R}^{n}\right)$ quando $n=3,4$.

Quando $B=T^{2}$, temos $\pi_{1}(M)=\left\langle a_{1}, b_{1} \mid\left[a_{1}, b_{1}\right]\right\rangle \times\left\langle c \mid c^{2}\right\rangle$ (como no exemplo 3.24), donde obtemos:

Proposição 4.12. Sejam $\xi_{M}=\left(E, T^{2}, \mathbb{R} P^{2}, p\right)$ o fibrado trivial e $0 \neq[\varphi] \in H^{1}\left(M ; \mathbb{Z}_{2}\right)$ tal que $\varphi(c)=\overline{1}$ e $\varphi(x)=\overline{1}$ para algum gerador $x$ de $\pi_{1}\left(T^{2}\right)$. Então, a propriedade de Borsuk-Ulam vale para $\left(M_{\varphi}, \tau_{\varphi} ; \mathbb{R}^{3}\right)$ e não vale para $\left(M_{\varphi}, \tau_{\varphi} ; \mathbb{R}^{4}\right)$.

Demonstração. Para $\varphi=\varphi_{1}$, no Exemplo 3.24, vimos que $M_{\varphi} \simeq \mathbb{S}^{1} \times\left(\mathbb{S}^{1} \widetilde{\times} \mathbb{S}^{2}\right)$ e a involução livre sobre $\mathbb{S}^{1} \times\left(\mathbb{S}^{1} \widetilde{\times} \mathbb{S}^{2}\right)$ é da forma diagonal $i d \times \tau$, onde $\tau$ é uma involução livre sobre $\mathbb{S}^{1} \widetilde{\times} \mathbb{S}^{2}$. Por [1], $\tau$ é única e tal que a propriedade de Borsuk-Ulam vale para $\left(\mathbb{S}^{1} \widetilde{\times} \mathbb{S}^{2}, \tau ; \mathbb{R}^{3}\right)$. Logo, pela Proposição 2.3, a propriedade de Borsuk-Ulam vale para $\left(\mathbb{S}^{1} \times\left(\mathbb{S}^{1} \widetilde{\times} \mathbb{S}^{2}\right), i d \times \tau ; \mathbb{R}^{3}\right)$, e consequentemente, pelo Lema 4.2 , ela também vale para $\left(M_{\varphi}, \tau_{\varphi} ; \mathbb{R}^{3}\right)$. Agora, como a propriedade de Borsuk-Ulam não vale para $\left(\mathbb{S}^{1} \widetilde{\times} \mathbb{S}^{2}, \tau ; \mathbb{R}^{4}\right)$, pois $\operatorname{dim}\left(\mathbb{S}^{1} \widetilde{\times} \mathbb{S}^{2}\right)=3$ (Lema $\left.1.18(3)\right)$, da Proposição 2.3, segue que ela também não vale para $\left(\mathbb{S}^{1} \times\left(\mathbb{S}^{1} \widetilde{\times} \mathbb{S}^{2}\right), i d \times \tau ; \mathbb{R}^{4}\right)$, e portanto também não vale para $\left(M_{\varphi}, \tau_{\varphi} ; \mathbb{R}^{4}\right)$ (Lema 4.2. Para $\varphi=\varphi_{2}, \varphi_{3}$, como existe isomorfismo $\nu: \pi_{1}\left(E_{\varphi_{i}} / \tau_{\varphi_{i}}^{\prime}\right) \rightarrow \pi_{1}\left(E_{\varphi_{1}} / \tau_{\varphi_{1}}^{\prime}\right)$ tal que o seguinte diagrama comuta

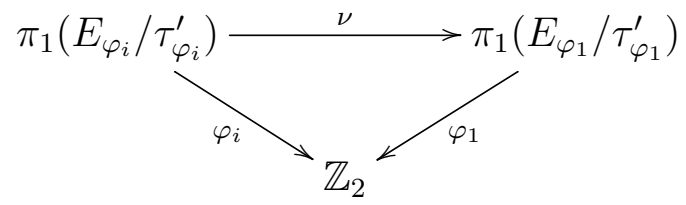

$(i=2,3)$, conseguimos equivalência de homotopia $h: E_{\varphi_{i}} / \tau_{\varphi_{i}}^{\prime} \rightarrow E_{\varphi_{1}} / \tau_{\varphi_{1}}^{\prime}$ tal que $h^{*}\left(\left[\varphi_{1}\right]\right)=\left[\varphi_{i}\right], i=2,3$. Portanto, para $\varphi=\varphi_{2}, \varphi_{3}$, o resultado segue do Corolário 1.17

\section{Observação 4.13.}

(1) Se $\xi_{M}$ é o $\mathbb{R} P^{2}$-fibrado trivial sobre $B$ (superfície fechada $K(\pi, 1)$ ) tal que $\varphi(c)=\overline{1}$ e $\varphi(x)=\overline{0}$, para todo gerador $x$ de $\pi_{1}(B)$, segue que a propriedade de Borsuk-Ulam 
não vale para $\left(M_{\varphi}, \tau_{\varphi} ; \mathbb{R}^{n}\right)$ quando $n=3,4$, pois a involução $\tau_{\varphi}^{\prime}$ sobre $E_{\varphi}$ é a diagonal, conforme vimos na Observação 3.27 .

(2) Com exceção da proposição anterior e do item (1) desta observação, ainda não sabemos sobre a validade da propriedade de Borsuk-Ulam para $\left(M_{\varphi}, \tau_{\varphi} ; \mathbb{R}^{n}\right)$ quando $n=3,4$ e $\varphi(c)=\overline{1}$.

\subsection{3 $F$ é $K(\pi, 1)$ e $B=\mathbb{S}^{2}$}

Seja $\xi_{M}=\left(E, \mathbb{S}^{2}, F, p\right)$, com $F$ uma superfície fechada $K(\pi, 1)$. Dividimos em três casos, como segue:

\section{(I) $F \neq T^{2}, K b$}

Como vimos no capítulo anterior, $\xi_{M}$ é único, a menos de equivalência de fibrados, quando $F \neq T^{2}, K b$, isto é, $M \simeq \mathbb{S}^{2} \times F$. Daí, $\pi_{1}(M) \cong \pi_{1}(F)$, e dada $0 \neq[\varphi] \in$ $H^{1}\left(M ; \mathbb{Z}_{2}\right.$ ), sabemos que $\xi_{M_{\varphi}}$ é um $F_{\varphi}$-fibrado sobre $\mathbb{S}^{2}$ (Teorema 3.5), que também é único, uma vez que $F_{\varphi}$ é $K(\pi, 1)$, diferente de $T^{2}$ e $K b$, ou seja, $M_{\varphi} \simeq \mathbb{S}^{2} \times F_{\varphi}$. Portanto, não há necessidade de analisarmos a propriedade de Borsuk-Ulam para $\left(M_{\varphi}, \tau_{\varphi} ; \mathbb{R}^{n}\right)$, uma vez que a mesma já foi analisada na Subseção 4.3.1.

(II) $F=T^{2}$

No caso em que $\xi_{M}=\left(E, \mathbb{S}^{2}, T^{2}, p\right)$, da sequência $(3.1)$, temos

$$
\cdots \longrightarrow \mathbb{Z} \stackrel{\partial}{\longrightarrow} \mathbb{Z}^{2} \longrightarrow \pi_{1}(M) \longrightarrow 1
$$

onde $\partial$ é o homomorfismo conectante. Assim,

- se $\partial=1$, então $\pi_{1}(M) \cong \mathbb{Z}^{2}$;

- se $\partial \neq 1$, então $\pi_{1}(M) \cong \mathbb{Z} \oplus \mathbb{Z}_{n}=\left\langle x, y \mid[x, y], y^{n}\right\rangle$, para algum $n>0$. ([19, Teorema $5.19(3)]$.

Proposição 4.14. Sejam $\xi_{M}=\left(E, \mathbb{S}^{2}, T^{2}, p\right)$ e $0 \neq[\varphi] \in H^{1}\left(M ; \mathbb{Z}_{2}\right)$. De acordo com a notação anterior,

(i) se $\partial=1$, então a propriedade de Borsuk-Ulam não vale para $\left(M_{\varphi}, \tau_{\varphi} ; \mathbb{R}^{2}\right)$.

(ii) se $\partial \neq 1$, então

- a propriedade de Borsuk-Ulam não vale para $\left(M_{\varphi}, \tau_{\varphi} ; \mathbb{R}^{2}\right)$, se $\varphi(y)=\overline{0}$;

- a propriedade de Borsuk-Ulam vale para $\left(M_{\varphi}, \tau_{\varphi} ; \mathbb{R}^{2}\right)$, se $\varphi(y)=\overline{1}$. 
Demonstração. O resultado segue da verificação da fatoração do diagrama (*), do Teorema 1.20 .

Observação 4.15. Nas hipóteses da proposição anterior,

(1) se $\partial=1$ ou $\partial \neq 1$ e $\varphi(y)=\overline{0}$, a propriedade de Borsuk-Ulam não vale para $\left(M_{\varphi}, \tau_{\varphi} ; \mathbb{R}^{n}\right)$ quando $n=3,4$;

(2) se $\partial \neq 1$ e $\varphi(y)=\overline{1}$, ainda não podemos dizer sobre a validade da propriedade para $\left(M_{\varphi}, \tau_{\varphi} ; \mathbb{R}^{n}\right)$ quando $n=3,4$.

(III) $F=K \boldsymbol{b}$

Para $\xi_{M}=\left(E, \mathbb{S}^{2}, K b, p\right)$, da sequência 3.1 temos

$$
\cdots \longrightarrow \mathbb{Z} \stackrel{\partial}{\longrightarrow} \pi_{1}(K b) \longrightarrow \pi_{1}(M) \longrightarrow 1 \text {. }
$$

Assim,

- se $\partial=1$, então $\pi_{1}(M) \cong\left\langle x, y \mid x y x y^{-1}\right\rangle$;

- se $\partial \neq 1$, então $\pi_{1}(M) \cong\left\langle x, y \mid x y x y^{-1}, y^{2 n}\right\rangle$, para algum $n>0$. ([19, Teorema $5.19(4)]$.

Proposição 4.16. Sejam $\xi_{M}=\left(E, \mathbb{S}^{2}, K b, p\right)$ e $0 \neq[\varphi] \in H^{1}\left(M ; \mathbb{Z}_{2}\right)$. De acordo com a notação anterior,

(i) para $\partial=1$, a propriedade de Borsuk-Ulam vale para $\left(M_{\varphi}, \tau_{\varphi} ; \mathbb{R}^{2}\right)$ se, e somente se, $\varphi(x)=\overline{1}$.

(ii) se $\partial \neq 1$, então a propriedade de Borsuk-Ulam vale para $\left(M_{\varphi}, \tau_{\varphi} ; \mathbb{R}^{2}\right)$.

Demonstração. O resultado segue da verificação da fatoração do diagrama (*), do Teorema 1.20 .

Observação 4.17. Nas hipóteses da proposição anterior,

(1) se $\partial=1$ e $\varphi(x)=\overline{0}$, então a propriedade de Borsuk-Ulam não vale para $\left(M_{\varphi}, \tau_{\varphi} ; \mathbb{R}^{n}\right)$ quando $n=3,4$.

(2) se $\partial=1$ e $\varphi(x)=\overline{1}$ ou $\partial \neq 1$, ainda não sabemos sobre a validade da propriedade para $\left(M_{\varphi}, \tau_{\varphi} ; \mathbb{R}^{n}\right)$ quando $n=3,4$. 


\subsection{4 $F$ é $K(\pi, 1)$ e $B=\mathbb{R} P^{2}$}

Seja $\xi_{M}=\left(E, \mathbb{R} P^{2}, F, p\right)$, com $F$ uma superfície fechada $K(\pi, 1)$. Da sequência (3.1), temos a sequência exata

$$
1 \longrightarrow \pi_{2}(M) \longrightarrow \mathbb{Z} \stackrel{\partial}{\longrightarrow} \pi_{1}(F) \longrightarrow \pi_{1}(M) \stackrel{p_{\#}}{\longrightarrow} \mathbb{Z}_{2} \longrightarrow 1
$$

Dividimos a análise nos seguintes casos:

(I) $\chi(F)<0$ ou $\chi(F)=0$ e $\partial=1$

Definição 4.18. Um grupo $G$ é chamado um grupo de superfície, se existe uma superfície fechada $S$ de gênus maior ou igual a um tal que $G \cong \pi_{1}(S)$.

Para o fibrado $\xi_{M}$ em questão, de (4.3) e [16, Corolário IV.4], temos a sequência exata curta

$$
1 \longrightarrow \pi_{1}(F) \longrightarrow \pi_{1}(M) \stackrel{p_{\#}}{\longrightarrow} \mathbb{Z}_{2} \longrightarrow 1
$$

donde segue o seguinte lema:

Lema 4.19. Seja $\xi_{M}=\left(E, \mathbb{R} P^{2}, F, p\right)$ tal que $\chi(F)<0$ ou $\chi(F)=0$ e $\partial=1$ (homomorfismo conectante). Se $\pi_{1}(M)$ é livre de torção, então ele é um grupo de superfície.

Demonstração. De acordo com nossas hipóteses, da sequência (4.4), temos $\pi_{1}(M)$ livre de torção contendo um grupo de superfície, $\pi_{1}(F)$, como subgrupo de índice dois. Logo, por [10, Corolário 2], existe superfície fechada $S$, diferente de $\mathbb{R} P^{2}$, tal que $\pi_{1}(M) \cong \pi_{1}(S)$.

Desta forma, de (4.1) e (4.2), obtemos o seguinte resultado:

Proposição 4.20. Sejam $\xi_{M}=\left(E, \mathbb{R} P^{2}, F, p\right)$, com $\chi(F)<0$ ou $\chi(F)=0$ e $\partial=1$, e $0 \neq[\varphi] \in H^{1}\left(M ; \mathbb{Z}_{2}\right)$. Se $\pi_{1}(M)$ é livre de torção, então, com a notação anterior, a propriedade de Borsuk-Ulam vale para $\left(M_{\varphi}, \tau_{\varphi} ; \mathbb{R}^{2}\right)$ se, e somente se, $S$ é não orientável $\left(\neq \mathbb{R} P^{2}\right)$ e $\varphi$ envia uma quantidade ímpar de geradores de $\pi_{1}(S)$ em $\overline{1}$.

Demonstração. Análoga à prova da Proposição 4.8 .

No caso de $\pi_{1}(M)$ ter torção, temos o seguinte lema:

Lema 4.21. Se $\xi_{M}=\left(E, \mathbb{R} P^{2}, F, p\right)$, com $\chi(F)<0$ ou $\chi(F)=0$ e $\partial=1$, e $x \in \pi_{1}(M)$ é um elemento de torção, então sua ordem de torção é dois. 
Demonstração. Suponhamos que $x \in \pi_{1}(M)$ é um elemento de torção. Da sequência (4.4), $p_{\#}(x) \neq \overline{0}$, pois se $p_{\#}(x)=\overline{0}$, temos $x \in \pi_{1}(F)$, o que contradiz o fato de que $\pi_{1}(F)$ é livre de torção. Logo, $p_{\#}(x)=\overline{1}$, e assim

(i) suponhamos que a ordem de torção de $x$ é $2 m+1(m$ inteiro $\geq 1)$. Então, $p_{\#}(x)^{2 m+1}=$ $\overline{0}$, o que implica em $p_{\#}(x)=\overline{0}$, e isto é uma contradição, ou seja, a ordem de torção de $x$ não pode ser ímpar;

(ii) se $x^{2 m+2}=1(m$ inteiro $\geq 1)$, então $p_{\#}(x)^{2 m+2}=\left(p_{\#}(x)^{2}\right)^{m+1}=\overline{0}$. Como $p_{\#}(x)^{2}=\overline{0}$, temos $x^{2} \in \pi_{1}(F)$ com ordem $m+1$, o que é uma contradição, e assim a ordem de torção de $x$ não pode ser par maior do que dois.

Portanto, se $\pi_{1}(M)$ tiver torção ela só poderá ser de ordem dois.

Proposição 4.22. Sejam $\xi_{M}=\left(E, \mathbb{R} P^{2}, F, p\right)$, com $\chi(F)<0$ ou $\chi(F)=0$ e $\partial=1$, e $0 \neq[\varphi] \in H^{1}\left(M ; \mathbb{Z}_{2}\right)$ tal que $\varphi(x)=\overline{1}$ para algum elemento de torção $x \in \pi_{1}(M)$. Então, a propriedade de Borsuk-Ulam vale para $\left(M_{\varphi}, \tau_{\varphi} ; \mathbb{R}^{2}\right)$.

Demonstração. Pelo lema anterior, o resultado segue da análise da fatoração do diagrama $(*)$.

Observação 4.23. Seja $\xi_{M}$ um $F$-fibrado sobre $\mathbb{R} P^{2}$ como em (I),

(1) a propriedade de Borsuk-Ulam não vale para $\left(M_{\varphi}, \tau_{\varphi} ; \mathbb{R}^{n}\right)$ quando $n=3,4$, nos seguintes casos:

- $\varphi=p_{\#}$. De fato, notemos que $p_{\#}\left(\pi_{1}\left(E_{\varphi}\right)\right)=1$, e assim a conclusão segue do Teorema 4.3. Nesta situação temos $E_{\varphi} \approx \mathbb{S}^{2} \times F([19$, Teorema 5.20(3)]);

- $\varphi \neq p_{\#}, \pi_{1}(M)$ livre de torção, com $\pi_{1}(M) \cong \pi_{1}(S)$ e

(a) $S$ é orientável; ou

(b) $S$ é não orientável e $\varphi$ envia uma quantidade par de elementos de $\pi_{1}(S)$ em $\overline{1}$;

(2) Não sabemos sobre a validade da propriedade de Borsuk-Ulam para $\left(M_{\varphi}, \tau_{\varphi} ; \mathbb{R}^{n}\right)$ quando $n=3,4$, nos casos não contemplados no item (1) desta observação.

(II) $\partial \neq 1$

A condição $\partial \neq 1$ implica que $\chi(F)=0$. Assim, para $F=K b$, obtemos o seguinte resultado:

Proposição 4.24. Sejam $\xi_{M}=\left(E, \mathbb{R} P^{2}, K b, p\right), 0 \neq[\varphi] \in H^{1}\left(M ; \mathbb{Z}_{2}\right)$ e $\partial \neq 1$. Então, a propriedade de Borsuk-Ulam vale para $\left(M_{\varphi}, \tau_{\varphi} ; \mathbb{R}^{2}\right)$. 
Demonstração. Por [19, Seção 11.6],

$$
\pi_{1}(M)=\left\langle t, u, w \mid u w u^{-1}=w^{-1}, u^{n}, t u t^{-1}=u^{-1}, t w t^{-1}=u^{a} w^{\epsilon}, t^{2}=u^{b} w^{c}\right\rangle,
$$

onde $n$ é par maior que zero, $a, b, c$ inteiros $(\geq 0)$ e $\epsilon= \pm 1$. Assim, suponhamos que exista homomorfismo $\eta: \pi_{1}(M) \rightarrow \mathbb{Z}$ tal que o diagrama $(*)$, do Teorema 1.20 , se fatore. Das relações $u^{n}=1, u w u^{-1}=w^{-1}$ e $t^{2}=u^{b} w^{c}$, temos $\eta(t)=\eta(u)=\eta(w)=0$, o que contradiz o fato de que pelo menos um dos geradores de $\pi_{1}(M)$ é enviado em $\overline{1}$ por $\varphi$.

Observação 4.25. Quando $\partial \neq 1$, ainda não sabemos sobre a validade da propriedade de Borsuk-Ulam para $\left(M_{\varphi}, \tau_{\varphi} ; \mathbb{R}^{n}\right)$ nas seguintes situações:

- $F=K b$ e $n=3,4$;

- $F=T^{2}$ e $n=2,3,4$.

\subsection{Considerações sobre a subfamília $\mathcal{F}_{3}$}

Com exceção dos produtos de superfícies fechadas (ambas $K(\pi, 1)$ ), a dificuldade de determinarmos, por exemplo, uma apresentação para o grupo fundamental dos demais elementos da subfamília $\mathcal{F}_{3}$, tornou inviável, até o momento, darmos uma resposta sobre a validade da propriedade de Borsuk-Ulam para os revestimentos duplos dos elementos desta subfamília. No entanto, alguns casos particulares de elementos de $\mathcal{F}_{3}$ equipados com involuções livres, serão explorados nos Capítulos 5 e 6 . 


\section{Capítulo 5}

\section{PBU para $\left(T^{4}, \tau ; \mathbb{R}^{n}\right)$}

Dizemos que um grupo $\pi$ é um grupo de n-variedade "flat" se ele é livre de torção e tem um subgrupo normal de índice finito, que é isomorfo a $\mathbb{Z}^{n}$. Estas são condições necessárias e suficientes para que $\pi$ seja o grupo fundamental de uma $n$-variedade riemanniana fechada "flat". Em [18], são apresentados todos os grupos de 4-variedade "flat", cujo número de tais grupos é 74, ou seja, são conhecidas todas as 4-variedades fechadas que são revestidas finitamente por $T^{4}$. Um grupo de 4-variedade "flat" $\pi$ é orientável se, e somente se, a 4-variedade "flat" correspondente é orientável (para maiores detalhes vide [18]). Então, nas duas próximas seções, usando [18], identificaremos as 4-variedades "flat" orientáveis e não orientáveis que são revestidas duplamente por $T^{4}$, e estudaremos as involuções livres sobre $T^{4}$. Finalizaremos com o estudo da propriedade de Borsuk-Ulam para $\left(T^{4}, \tau ; \mathbb{R}^{n}\right)$. Observemos que $T^{4}$ é o espaço total de um $T^{2}$-fibrado sobre $T^{2}$, ou seja, $T^{4}$ é um elemento da subfamília $\mathcal{F}_{3}$.

\subsection{4-variedades "flat" orientáveis}

De acordo com [18], os grupos de 4-variedade "flat" orientáveis $\pi$ tais que $\pi / \mathbb{Z}^{4} \cong \mathbb{Z}_{2}$, são dados na sequência:

(I) O primeiro grupo orientável é o produto semidireto de $\mathbb{Z}^{3}$ por $\mathbb{Z}$, pela ação determinada pela matriz

$$
T=\left(\begin{array}{ccc}
1 & 0 & 0 \\
0 & -1 & 0 \\
1 & 0 & -1
\end{array}\right),
$$


isto é, $\pi \cong \mathbb{Z}^{3} \rtimes_{T} \mathbb{Z}$. Uma apresentação para $\pi$ é dada por

$$
\pi=\left\langle t, x, y, z \mid t x t^{-1}=x z,[y, t]^{\prime},[z, t]^{\prime},[x, y],[x, z],[y, z]\right\rangle
$$

onde $[u, v]^{\prime}=u v u v^{-1}$. Assim, denotemos por $\mathcal{M}_{O}^{1}$ a 4 -variedade "flat" orientável tal que $\pi_{1}\left(\mathcal{M}_{O}^{1}\right)=\pi$. Vejamos as involuções livres $\tau$ sobre $T^{4}$ tais que $T^{4} / \tau=\mathcal{M}_{O}^{1}$. Para isto, através do método de Reidemeister-Schreier, determinaremos quais das 4-variedades que revestem duplamente $\mathcal{M}_{O}^{1}$, correspondem a $T^{4}$. Sendo $\varphi$ um epimorfismo de $\pi_{1}\left(\mathcal{M}_{O}^{1}\right)$ em $\mathbb{Z}_{2}$, da relação $t x t^{-1}=x z$, de $(5.1)$, temos $\varphi(z)=\overline{0}$, e portanto $\varphi$ pode ser um dos sete epimorfismos listados na seguinte tabela:

\begin{tabular}{|l|l|l|l|l|}
\hline & $\boldsymbol{t}$ & $\boldsymbol{x}$ & $\boldsymbol{y}$ & $\boldsymbol{z}$ \\
\hline $\boldsymbol{\varphi}_{\mathbf{1}}$ & $\overline{1}$ & $\overline{0}$ & $\overline{0}$ & $\overline{0}$ \\
\hline $\boldsymbol{\varphi}_{\mathbf{2}}$ & $\overline{0}$ & $\overline{1}$ & $\overline{0}$ & $\overline{0}$ \\
\hline $\boldsymbol{\varphi}_{\mathbf{3}}$ & $\overline{0}$ & $\overline{0}$ & $\overline{1}$ & $\overline{0}$ \\
\hline $\boldsymbol{\varphi}_{\mathbf{4}}$ & $\overline{1}$ & $\overline{1}$ & $\overline{0}$ & $\overline{0}$ \\
\hline
\end{tabular}

\begin{tabular}{|l|l|l|l|l|}
\hline & $\boldsymbol{t}$ & $\boldsymbol{x}$ & $\boldsymbol{y}$ & $\boldsymbol{z}$ \\
\hline $\boldsymbol{\varphi}_{\mathbf{5}}$ & $\overline{1}$ & $\overline{0}$ & $\overline{1}$ & $\overline{0}$ \\
\hline $\boldsymbol{\varphi}_{\mathbf{6}}$ & $\overline{0}$ & $\overline{1}$ & $\overline{1}$ & $\overline{0}$ \\
\hline $\boldsymbol{\varphi}_{\mathbf{7}}$ & $\overline{1}$ & $\overline{1}$ & $\overline{1}$ & $\overline{0}$ \\
\hline
\end{tabular}

Tabela 5.1: Epimorfismos de $\pi_{1}\left(\mathcal{M}_{O}^{1}\right)$ em $\mathbb{Z}_{2}$.

Para cada $i=1, \ldots, 7$, determinaremos uma apresentação para $\operatorname{Ker}\left(\varphi_{i}\right)$ como segue:

- $\operatorname{Ker}\left(\varphi_{1}\right)$

Seja $\{1, t\}$ um sistema de Schreier. Daí, os geradores de $\operatorname{Ker}\left(\varphi_{1}\right)$ são:

$$
\begin{aligned}
& y_{11}=\varrho(1, t)=t \cdot(\bar{t})^{-1}=1 \\
& y_{12}=\varrho(t, t)=t t \cdot(\overline{t t})^{-1}=t^{2}, \\
& y_{21}=\varrho(1, x)=x \cdot(\bar{x})^{-1}=x, \\
& y_{22}=\varrho(t, x)=t x \cdot(\overline{t x})^{-1}=t x t^{-1}, \\
& y_{31}=\varrho(1, y)=y \cdot(\bar{y})^{-1}=y, \\
& y_{32}=\varrho(t, y)=t y \cdot(\overline{t y})^{-1}=t y t^{-1}, \\
& y_{41}=\varrho(1, z)=z \cdot(\bar{z})^{-1}=z, \\
& y_{42}=\varrho(t, z)=t z \cdot(\overline{t z})^{-1}=t z t^{-1},
\end{aligned}
$$

e as relações são:
(1) $y_{21}^{-1} y_{22} y_{41}^{-1}=1$,
(2) $\left.y_{22}^{-1} y_{12} y_{21} y_{12}^{-1} y_{42}^{-1}\right]=1$,
(3) $y_{31} y_{32}=1$
(4) $y_{32} y_{12} y_{31} y_{12}^{-1}=1$,
(5) $y_{41} y_{42}=1$, 
(6) $y_{42} y_{12} y 41 y_{12}^{-1}=1$,

(7) $\left[y_{21}, y_{31}\right]=1$,

(8) $\left[y_{22}, y_{32}\right]=1$,

(9) $\left[y_{21}, y_{41}\right]=1$,

(10) $\left[y_{22}, y_{42}\right]=1$,

(11) $\left[y_{31}, y_{41}\right]=1$,

(12) $\left[y_{32}, y_{42}\right]=1$.

Com a notação $y_{1}=y_{12}, y_{2}=y_{21}, y_{3}=y_{31}$ e $y_{4}=y_{41}$, temos

$$
\left.\operatorname{Ker}\left(\varphi_{1}\right)=\left\langle y_{1}, y_{2}, y_{3}, y_{4}\right| y_{1}, y_{2}, y_{3}, y_{4} \text { comutam }\right\rangle \cong \mathbb{Z}^{4},
$$

ou seja, o revestimento duplo de $\mathcal{M}_{O}^{1}$ associado a $\varphi_{1}$ é $T^{4}$.

De modo análogo ao que foi feito para $\varphi_{1}$, para os demais epimorfismos, temos:

- $\operatorname{Ker}\left(\varphi_{2}\right)=\left\langle y_{1}, y_{2}, y_{3}, y_{4} \mid\left[y_{2}, y_{1}\right] y_{4}^{2},\left[y_{3}, y_{1}\right]^{\prime},\left[y_{4}, y_{1}\right]^{\prime},\left[y_{2}, y_{3}\right],\left[y_{2}, y_{4}\right],\left[y_{3}, y_{4}\right]\right\rangle$, onde $y_{1}=t$, $y_{2}=x^{2}, y_{3}=y$ e $y_{4}=z$.

- $\operatorname{Ker}\left(\varphi_{3}\right)=\left\langle y_{1}, y_{2}, y_{3}, y_{4} \mid y_{1} y_{2} y_{1}^{-1}=y_{2} y_{4},\left[y_{3}, y_{1}\right]^{\prime},\left[y_{4}, y_{1}\right]^{\prime},\left[y_{2}, y_{3}\right],\left[y_{2}, y_{4}\right],\left[y_{3}, y_{4}\right]\right\rangle$, onde $y_{1}=t, y_{2}=x, y_{3}=y^{2}$ e $y_{4}=z$.

- $\operatorname{Ker}\left(\varphi_{4}\right)=\left\langle y_{1}, y_{2}, y_{3}, y_{4} \mid y_{1} y_{2} y_{4}^{-1}=y_{2} y_{4}^{-1} y_{1},\left[y_{1}, y_{3}\right],\left[y_{1}, y_{4}\right],\left[y_{3}, y_{2}\right]^{\prime},\left[y_{4}, y_{2}\right]^{\prime},\left[y_{3}, y_{4}\right]\right\rangle$, onde $y_{1}=t, y_{2}=x t^{-1}, y_{3}=y$ e $y_{4}=z$.

- $\operatorname{Ker}\left(\varphi_{5}\right)=\left\langle y_{1}, y_{2}, y_{3}, y_{4} \mid y_{1} y_{2} y_{1}^{-1}=y_{2} y_{4},\left[y_{3}, y_{1}\right]^{\prime},\left[y_{4}, y_{1}\right]^{\prime},\left[y_{2}, y_{3}\right],\left[y_{2}, y_{4}\right],\left[y_{3}, y_{4}\right]\right\rangle$, onde $y_{1}=t y^{-1}, y_{2}=x, y_{3}=y^{2}$ e $y_{4}=z$.

- $\operatorname{Ker}\left(\varphi_{6}\right)=\left\langle y_{1}, y_{2}, y_{3}, y_{4} \mid\left[y_{2}, y_{1}\right] y_{4}^{2},\left[y_{3}, y_{1}\right]^{\prime} y_{2} y_{4},\left[y_{4}, y_{1}\right]^{\prime},\left[y_{2}, y_{3}\right],\left[y_{2}, y_{4}\right],\left[y_{3}, y_{4}\right]\right\rangle$, onde $y_{1}=$ $t, y_{2}=x^{2}, y_{3}=y x^{-1}$ e $y_{4}=z$.

- $\operatorname{Ker}\left(\varphi_{7}\right)=\left\langle y_{1}, y_{2}, y_{3}, y_{4} \mid y_{1} y_{2} y_{1}^{-1}=y_{2} y_{3} y_{4},\left[y_{3}, y_{1}\right]^{\prime},\left[y_{4}, y_{1}\right]^{\prime},\left[y_{2}, y_{3}\right],\left[y_{2}, y_{4}\right],\left[y_{3}, y_{4}\right]\right\rangle$, onde $y_{1}=t y^{-1}, y_{2}=x y^{-1}, y_{3}=y^{2}$ e $y_{4}=z$.

Como $\operatorname{Ker}\left(\varphi_{i}\right)_{a b} \nsucceq \mathbb{Z}^{4}$, para $i=2, \ldots, 7$, segue que nenhum dos revestimentos duplos de $\mathcal{M}_{O}^{1}$ associados a estes $\varphi_{i}$ 's corresponde a $T^{4}$. Portanto, existe apenas uma involução livre sobre $T^{4}$, que denotamos por $\tau_{1}$, tal que $T^{4} / \tau_{1}=\mathcal{M}_{O}^{1}$.

(II) O segundo grupo orientável é tal que

$$
\pi \cong \mathbb{Z}^{3} \rtimes_{T} \mathbb{Z}
$$

onde a ação é dada pela matriz

$$
T=\left(\begin{array}{ccc}
1 & 0 & 0 \\
0 & -1 & 0 \\
0 & 0 & -1
\end{array}\right) .
$$


Assim,

$$
\pi=\left\langle t, x, y, z \mid[t, x],[y, t]^{\prime},[z, t]^{\prime},[x, y],[x, z],[y, z]\right\rangle .
$$

Denotemos por $\mathcal{M}_{O}^{2}$ a 4-variedade "flat" orientável tal que $\pi_{1}\left(\mathcal{M}_{O}^{2}\right)=\pi$. Daí, os epimorfismos de $\pi_{1}\left(\mathcal{M}_{O}^{2}\right)$ em $\mathbb{Z}_{2}$ são dados na seguinte tabela.

\begin{tabular}{|l|l|l|l|l|}
\hline & $\boldsymbol{t}$ & $\boldsymbol{x}$ & $\boldsymbol{y}$ & $\boldsymbol{z}$ \\
\hline $\boldsymbol{\varphi}_{\mathbf{1}}$ & $\overline{1}$ & $\overline{0}$ & $\overline{0}$ & $\overline{0}$ \\
\hline $\boldsymbol{\varphi}_{\mathbf{2}}$ & $\overline{0}$ & $\overline{1}$ & $\overline{0}$ & $\overline{0}$ \\
\hline $\boldsymbol{\varphi}_{\mathbf{3}}$ & $\overline{0}$ & $\overline{0}$ & $\overline{1}$ & $\overline{0}$ \\
\hline $\boldsymbol{\varphi}_{\mathbf{4}}$ & $\overline{0}$ & $\overline{0}$ & $\overline{0}$ & $\overline{1}$ \\
\hline $\boldsymbol{\varphi}_{\mathbf{5}}$ & $\overline{1}$ & $\overline{1}$ & $\overline{0}$ & $\overline{0}$ \\
\hline
\end{tabular}

\begin{tabular}{|l|c|c|c|c|}
\hline & $\boldsymbol{t}$ & $\boldsymbol{x}$ & $\boldsymbol{y}$ & $\boldsymbol{z}$ \\
\hline $\boldsymbol{\varphi}_{\mathbf{6}}$ & $\overline{1}$ & $\overline{0}$ & $\overline{1}$ & $\overline{0}$ \\
\hline $\boldsymbol{\varphi}_{\mathbf{7}}$ & $\overline{1}$ & $\overline{0}$ & $\overline{0}$ & $\overline{1}$ \\
\hline $\boldsymbol{\varphi}_{\mathbf{8}}$ & $\overline{0}$ & $\overline{1}$ & $\overline{1}$ & $\overline{0}$ \\
\hline $\boldsymbol{\varphi}_{\mathbf{9}}$ & $\overline{0}$ & $\overline{1}$ & $\overline{0}$ & $\overline{1}$ \\
\hline $\boldsymbol{\varphi}_{\mathbf{1 0}}$ & $\overline{0}$ & $\overline{0}$ & $\overline{1}$ & $\overline{1}$ \\
\hline
\end{tabular}

\begin{tabular}{|l|l|l|l|l|}
\hline & $\boldsymbol{t}$ & $\boldsymbol{x}$ & $\boldsymbol{y}$ & $\boldsymbol{z}$ \\
\hline $\boldsymbol{\varphi}_{\mathbf{1 1}}$ & $\overline{1}$ & $\overline{1}$ & $\overline{1}$ & $\overline{0}$ \\
\hline $\boldsymbol{\varphi}_{\mathbf{1 2}}$ & $\overline{1}$ & $\overline{1}$ & $\overline{0}$ & $\overline{1}$ \\
\hline $\boldsymbol{\varphi}_{\mathbf{1 3}}$ & $\overline{1}$ & $\overline{0}$ & $\overline{1}$ & $\overline{1}$ \\
\hline $\boldsymbol{\varphi}_{\mathbf{1 4}}$ & $\overline{0}$ & $\overline{1}$ & $\overline{1}$ & $\overline{1}$ \\
\hline $\boldsymbol{\varphi}_{\mathbf{1 5}}$ & $\overline{1}$ & $\overline{1}$ & $\overline{1}$ & $\overline{1}$ \\
\hline
\end{tabular}

Tabela 5.2: Epimorfismos de $\pi_{1}\left(\mathcal{M}_{O}^{2}\right)$ em $\mathbb{Z}_{2}$.

Procedendo como em (I), temos:

- $\operatorname{Ker}\left(\varphi_{1}\right)=\left\langle y_{1}, y_{2}, y_{3}, y_{4}\right| y_{1}, y_{2}, y_{3}, y_{4}$ comutam $\rangle \cong \mathbb{Z}^{4}$, onde $y_{1}=t^{2}, y_{2}=x, y_{3}=y$, $y_{4}=z$.

- $\operatorname{Ker}\left(\varphi_{2}\right)=\left\langle y_{1}, y_{2}, y_{3}, y_{4} \mid\left[y_{1}, y_{2}\right],\left[y_{3}, y_{1}\right]^{\prime},\left[y_{4}, y_{1}\right]^{\prime},\left[y_{2}, y_{3}\right],\left[y_{2}, y_{4}\right],\left[y_{3}, y_{4}\right]\right\rangle$, onde $y_{1}=t$, $y_{2}=x^{2}, y_{3}=y, y_{4}=z$.

- $\operatorname{Ker}\left(\varphi_{3}\right)=\left\langle y_{1}, y_{2}, y_{3}, y_{4} \mid\left[y_{2}, y_{1}\right],\left[y_{3}, y_{1}\right]^{\prime},\left[y_{4}, y_{1}\right]^{\prime},\left[y_{2}, y_{3}\right],\left[y_{2}, y_{4}\right],\left[y_{3}, y_{4}\right]\right\rangle$, onde $y_{1}=t$, $y_{2}=x, y_{3}=y^{2}, y_{4}=z$.

- $\operatorname{Ker}\left(\varphi_{4}\right)=\left\langle y_{1}, y_{2}, y_{3}, y_{4} \mid\left[y_{2}, y_{1}\right],\left[y_{3}, y_{1}\right]^{\prime},\left[y_{4}, y_{1}\right]^{\prime},\left[y_{2}, y_{3}\right],\left[y_{2}, y_{4}\right],\left[y_{3}, y_{4}\right]\right\rangle$, onde $y_{1}=t$, $y_{2}=x, y_{3}=y, y_{4}=z^{2}$.

- $\operatorname{Ker}\left(\varphi_{5}\right)=\left\langle y_{1}, y_{2}, y_{3}, y_{4} \mid\left[y_{1}, y_{2}\right],\left[y_{1}, y_{3}\right],\left[y_{1}, y_{4}\right],\left[y_{3}, y_{2}\right]^{\prime},\left[y_{4}, y_{2}\right]^{\prime},\left[y_{3}, y_{4}\right]\right\rangle$, onde $y_{1}=t^{2}$, $y_{2}=x t^{-1}, y_{3}=y, y_{4}=z$.

- $\operatorname{Ker}\left(\varphi_{6}\right)=\left\langle y_{1}, y_{2}, y_{3}, y_{4} \mid\left[y_{1}, y_{2}\right],\left[y_{3}, y_{1}\right]^{\prime},\left[y_{4}, y_{1}\right]^{\prime},\left[y_{2}, y_{3}\right],\left[y_{2}, y_{4}\right],\left[y_{3}, y_{4}\right]\right\rangle$, onde $y_{1}=t y^{-1}$, $y_{2}=x, y_{3}=y^{2}, y_{4}=z$.

- $\operatorname{Ker}\left(\varphi_{7}\right)=\left\langle y_{1}, y_{2}, y_{3}, y_{4} \mid\left[y_{1}, y_{2}\right],\left[y_{3}, y_{1}\right]^{\prime},\left[y_{4}, y_{1}\right]^{\prime},\left[y_{2}, y_{3}\right],\left[y_{2}, y_{4}\right],\left[y_{3}, y_{4}\right]\right\rangle$, onde $y_{1}=t z^{-1}$, $y_{2}=x, y_{3}=y, y_{4}=z^{2}$.

- $\operatorname{Ker}\left(\varphi_{8}\right)=\left\langle y_{1}, y_{2}, y_{3}, y_{4} \mid\left[y_{1}, y_{2}\right],\left[y_{3}, y_{1}\right]^{\prime} y_{2},\left[y_{4}, y_{1}\right]^{\prime},\left[y_{2}, y_{3}\right],\left[y_{2}, y_{4}\right],\left[y_{3}, y_{4}\right]\right\rangle$, onde $y_{1}=t$, $y_{2}=x^{2}, y_{3}=y x^{-1}, y_{4}=z$.

- $\operatorname{Ker}\left(\varphi_{9}\right)=\left\langle y_{1}, y_{2}, y_{3}, y_{4} \mid\left[y_{1}, y_{2}\right],\left[y_{3}, y_{1}\right]^{\prime},\left[y_{4}, y_{1}\right]^{\prime} y_{2},\left[y_{2}, y_{3}\right],\left[y_{2}, y_{4}\right],\left[y_{3}, y_{4}\right]\right\rangle$, onde $y_{1}=t$, $y_{2}=x^{2}, y_{3}=y, y_{4}=z x^{-1}$.

- $\operatorname{Ker}\left(\varphi_{10}\right)=\left\langle y_{1}, y_{2}, y_{3}, y_{4} \mid\left[y_{1}, y_{2}\right],\left[y_{3}, y_{1}\right]^{\prime},\left[y_{4}, y_{1}\right]^{\prime},\left[y_{2}, y_{3}\right],\left[y_{2}, y_{4}\right],\left[y_{3}, y_{4}\right]\right\rangle$, onde $y_{1}=t$, $y_{2}=x, y_{3}=y^{2}, y_{4}=z y^{-1}$. 
- $\operatorname{Ker}\left(\varphi_{11}\right)=\left\langle y_{1}, y_{2}, y_{3}, y_{4} \mid\left[y_{2}, y_{1}\right] y_{3},\left[y_{3}, y_{1}\right]^{\prime},\left[y_{4}, y_{1}\right]^{\prime},\left[y_{2}, y_{3}\right],\left[y_{2}, y_{4}\right],\left[y_{3}, y_{4}\right]\right\rangle$, onde $y_{1}=$ $t y^{-1}, y_{2}=x y^{-1}, y_{3}=y^{2}, y_{4}=z$.

- $\operatorname{Ker}\left(\varphi_{12}\right)=\left\langle y_{1}, y_{2}, y_{3}, y_{4} \mid\left[y_{1}, y_{2}\right],\left[y_{3}, y_{1}\right]^{\prime},\left[y_{4}, y_{1}\right]^{\prime} y_{2},\left[y_{2}, y_{3}\right],\left[y_{2}, y_{4}\right],\left[y_{3}, y_{4}\right]\right\rangle$, onde $y_{1}=$ $t x^{-1}, y_{2}=x^{2}, y_{3}=y, y_{4}=z x^{-1}$.

- $\operatorname{Ker}\left(\varphi_{13}\right)=\left\langle y_{1}, y_{2}, y_{3}, y_{4} \mid\left[y_{1}, y_{2}\right],\left[y_{3}, y_{1}\right]^{\prime},\left[y_{4}, y_{1}\right]^{\prime},\left[y_{2}, y_{3}\right],\left[y_{2}, y_{4}\right],\left[y_{3}, y_{4}\right]\right\rangle$, onde $y_{1}=t y^{-1}$, $y_{2}=x, y_{3}=y^{2}, y_{4}=z y^{-1}$.

- $\operatorname{Ker}\left(\varphi_{14}\right)=\left\langle y_{1}, y_{2}, y_{3}, y_{4} \mid\left[y_{2}, y_{1}\right] y_{3},\left[y_{3}, y_{1}\right]^{\prime},\left[y_{4}, y_{1}\right]^{\prime},\left[y_{2}, y_{3}\right],\left[y_{2}, y_{4}\right],\left[y_{3}, y_{4}\right]\right\rangle$, onde $y_{1}=t$, $y_{2}=x y^{-1}, y_{3}=y^{2}, y_{4}=z y^{-1}$.

- $\operatorname{Ker}\left(\varphi_{15}\right)=\left\langle y_{1}, y_{2}, y_{3}, y_{4} \mid\left[y_{2}, y_{1}\right] y_{3},\left[y_{3}, y_{1}\right]^{\prime},\left[y_{4}, y_{1}\right]^{\prime},\left[y_{2}, y_{3}\right],\left[y_{2}, y_{4}\right],\left[y_{3}, y_{4}\right]\right\rangle$, onde $y_{1}=$ $t y^{-1}, y_{2}=x y^{-1}, y_{3}=y^{2}, y_{4}=z y^{-1}$.

Como $\operatorname{Ker}\left(\varphi_{i}\right)_{a b} \neq \mathbb{Z}^{4}$, para $i=2, \ldots, 15$, existe apenas uma involução livre sobre $T^{4}$, que denotamos por $\tau_{2}$, tal que $T^{4} / \tau_{2}=\mathcal{M}_{O}^{2}$.

Observação 5.1. Além das duas variedades orientáveis apresentadas em (I) e (II), $T^{4}$ também é revestida duplamente por $T^{4}$, por exemplo, consideremos sobre $T^{4}=T^{2} \times T^{2}$ a involução diagonal livre $\tau \times i d$, onde $\tau$ é uma involução livre sobre $T^{2}$ tal que $T^{2} / \tau=T^{2}$ e $i d$ é a identidade sobre $T^{2}$. Assim, $T^{4} /(\tau \times i d)=T^{4}$.

\subsection{4-variedades "flat" não orientáveis}

Na sequência, apresentaremos os grupos de 4-variedade "flat" não orientáveis.

(I) O primeiro grupo de 4-variedade "flat" não orientável é tal que

$$
\pi \cong \mathbb{Z} \times B_{1}=\mathbb{Z}^{2} \times K=\left\langle t, x, y, z \mid[t, x],[t, y],[z, t]^{\prime},[x, y],[x, z],[y, z]\right\rangle,
$$

onde $B_{1}$ é o grupo de 3 -variedade "flat" não orientável e $K:=\pi_{1}(K b)$ ([18]). Também podemos ver $\pi$ como o produto semidireto, $\mathbb{Z}^{3} \rtimes_{T} \mathbb{Z}$, onde a ação é determinada pela matriz

$$
T=\left(\begin{array}{ccc}
1 & 0 & 0 \\
0 & 1 & 0 \\
0 & 0 & -1
\end{array}\right)
$$

Denotemos por $\mathcal{M}_{N}^{1}$ a 4-variedade "flat" não orientável tal que $\pi_{1}\left(\mathcal{M}_{N}^{1}\right)=\pi$. Logo, temos quinze epimorfismos de $\pi_{1}\left(\mathcal{M}_{N}^{1}\right)$ em $\mathbb{Z}_{2}$, que são como os da Tabela 5.2 . Na sequência, listamos uma apresentação para $\operatorname{Ker}\left(\varphi_{i}\right)$, para cada $i=1, \ldots, 15$ : 
- $\operatorname{Ker}\left(\varphi_{1}\right)=\left\langle y_{1}, y_{2}, y_{3}, y_{4}\right| y_{1}, y_{2}, y_{3}, y_{4}$ comutam $\rangle \cong \mathbb{Z}^{4}$, onde $y_{1}=t^{2}, y_{2}=x, y_{3}=y$, $y_{4}=z$

- $\operatorname{Ker}\left(\varphi_{2}\right)=\left\langle y_{1}, y_{2}, y_{3}, y_{4} \mid\left[y_{1}, y_{2}\right],\left[y_{1}, y_{3}\right],\left[y_{4}, y_{1}\right]^{\prime},\left[y_{2}, y_{3}\right],\left[y_{2}, y_{4}\right],\left[y_{3}, y_{4}\right]\right\rangle$, onde $y_{1}=t$, $y_{2}=x^{2}, y_{3}=y, y_{4}=z$.

- $\operatorname{Ker}\left(\varphi_{3}\right)=\left\langle y_{1}, y_{2}, y_{3}, y_{4} \mid\left[y_{2}, y_{1}\right],\left[y_{1}, y_{3}\right],\left[y_{4}, y_{1}\right]^{\prime},\left[y_{2}, y_{3}\right],\left[y_{2}, y_{4}\right],\left[y_{3}, y_{4}\right]\right\rangle$, onde $y_{1}=t$, $y_{2}=x, y_{3}=y^{2}, y_{4}=z$.

- $\operatorname{Ker}\left(\varphi_{4}\right)=\left\langle y_{1}, y_{2}, y_{3}, y_{4} \mid\left[y_{2}, y_{1}\right],\left[y_{1}, y_{3}\right],\left[y_{4}, y_{1}\right]^{\prime},\left[y_{2}, y_{3}\right],\left[y_{2}, y_{4}\right],\left[y_{3}, y_{4}\right]\right\rangle$, onde $y_{1}=t$, $y_{2}=x, y_{3}=y, y_{4}=z^{2}$.

- $\operatorname{Ker}\left(\varphi_{5}\right)=\left\langle y_{1}, y_{2}, y_{3}, y_{4} \mid\left[y_{1}, y_{2}\right],\left[y_{1}, y_{3}\right],\left[y_{1}, y_{4}\right],\left[y_{2}, y_{3}\right],\left[y_{4}, y_{2}\right]^{\prime},\left[y_{3}, y_{4}\right]\right\rangle$, onde $y_{1}=t^{2}$, $y_{2}=x t^{-1}, y_{3}=y, y_{4}=z$.

- $\operatorname{Ker}\left(\varphi_{6}\right)=\left\langle y_{1}, y_{2}, y_{3}, y_{4} \mid\left[y_{1}, y_{2}\right],\left[y_{1}, y_{3}\right],\left[y_{4}, y_{1}\right]^{\prime},\left[y_{2}, y_{3}\right],\left[y_{2}, y_{4}\right],\left[y_{3}, y_{4}\right]\right\rangle$, onde $y_{1}=t y^{-1}$, $y_{2}=x, y_{3}=y^{2}, y_{4}=z$.

- $\operatorname{Ker}\left(\varphi_{7}\right)=\left\langle y_{1}, y_{2}, y_{3}, y_{4} \mid\left[y_{1}, y_{2}\right],\left[y_{1}, y_{3}\right],\left[y_{4}, y_{1}\right]^{\prime},\left[y_{2}, y_{3}\right],\left[y_{2}, y_{4}\right],\left[y_{3}, y_{4}\right]\right\rangle$, onde $y_{1}=t z^{-1}$, $y_{2}=x, y_{3}=y, y_{4}=z^{2}$.

- $\operatorname{Ker}\left(\varphi_{8}\right)=\left\langle y_{1}, y_{2}, y_{3}, y_{4} \mid\left[y_{1}, y_{2}\right],\left[y_{1}, y_{3}\right],\left[y_{4}, y_{1}\right]^{\prime},\left[y_{2}, y_{3}\right],\left[y_{2}, y_{4}\right],\left[y_{3}, y_{4}\right]\right\rangle$, onde $y_{1}=t$, $y_{2}=x^{2}, y_{3}=y x^{-1}, y_{4}=z$.

- $\operatorname{Ker}\left(\varphi_{9}\right)=\left\langle y_{1}, y_{2}, y_{3}, y_{4} \mid\left[y_{1}, y_{2}\right],\left[y_{1}, y_{3}\right],\left[y_{4}, y_{1}\right]^{\prime} y_{2},\left[y_{2}, y_{3}\right],\left[y_{2}, y_{4}\right],\left[y_{3}, y_{4}\right]\right\rangle$, onde $y_{1}=t$, $y_{2}=x^{2}, y_{3}=y, y_{4}=z x^{-1}$.

- $\operatorname{Ker}\left(\varphi_{10}\right)=\left\langle y_{1}, y_{2}, y_{3}, y_{4} \mid\left[y_{1}, y_{2}\right],\left[y_{1}, y_{3}\right],\left[y_{4}, y_{1}\right]^{\prime} y_{3},\left[y_{2}, y_{3}\right],\left[y_{2}, y_{4}\right],\left[y_{3}, y_{4}\right]\right\rangle$, onde $y_{1}=t$, $y_{2}=x, y_{3}=y^{2}, y_{4}=z y^{-1}$.

- $\operatorname{Ker}\left(\varphi_{11}\right)=\left\langle y_{1}, y_{2}, y_{3}, y_{4} \mid\left[y_{1}, y_{2}\right],\left[y_{1}, y_{3}\right],\left[y_{4}, y_{1}\right]^{\prime},\left[y_{2}, y_{3}\right],\left[y_{2}, y_{4}\right],\left[y_{3}, y_{4}\right]\right\rangle$, onde $y_{1}=t y^{-1}$, $y_{2}=x y^{-1}, y_{3}=y^{2}, y_{4}=z$.

- $\operatorname{Ker}\left(\varphi_{12}\right)=\left\langle y_{1}, y_{2}, y_{3}, y_{4} \mid\left[y_{1}, y_{2}\right],\left[y_{1}, y_{3}\right],\left[y_{4}, y_{1}\right]^{\prime} y_{2},\left[y_{2}, y_{3}\right],\left[y_{2}, y_{4}\right],\left[y_{3}, y_{4}\right]\right\rangle$, onde $y_{1}=$ $t x^{-1}, y_{2}=x^{2}, y_{3}=y, y_{4}=z x^{-1}$.

- $\operatorname{Ker}\left(\varphi_{13}\right)=\left\langle y_{1}, y_{2}, y_{3}, y_{4} \mid\left[y_{1}, y_{2}\right],\left[y_{1}, y_{3}\right],\left[y_{4}, y_{1}\right]^{\prime} y_{3},\left[y_{2}, y_{3}\right],\left[y_{2}, y_{4}\right],\left[y_{3}, y_{4}\right]\right\rangle$, onde $y_{1}=$ $t y^{-1}, y_{2}=x, y_{3}=y^{2}, y_{4}=z y^{-1}$.

- $\operatorname{Ker}\left(\varphi_{14}\right)=\left\langle y_{1}, y_{2}, y_{3}, y_{4} \mid\left[y_{1}, y_{2}\right],\left[y_{1}, y_{3}\right],\left[y_{4}, y_{1}\right]^{\prime} y_{2},\left[y_{2}, y_{3}\right],\left[y_{2}, y_{4}\right],\left[y_{3}, y_{4}\right]\right\rangle$, onde $y_{1}=t$, $y_{2}=x^{2}, y_{3}=y x^{-1}, y_{4}=z x^{-1}$

- $\operatorname{Ker}\left(\varphi_{15}\right)=\left\langle y_{1}, y_{2}, y_{3}, y_{4} \mid\left[y_{1}, y_{2}\right],\left[y_{1}, y_{3}\right],\left[y_{4}, y_{1}\right]^{\prime} y_{3},\left[y_{2}, y_{3}\right],\left[y_{2}, y_{4}\right],\left[y_{3}, y_{4}\right]\right\rangle$, onde $y_{1}=$ $t y^{-1}, y_{2}=x y^{-1}, y_{3}=y^{2}, y_{4}=z y^{-1}$.

Analisando, cada uma destas apresentações, concluímos que dos quinze revestimentos duplos de $\mathcal{M}_{N}^{1}$ apenas um é $T^{4}$, e ele está associado ao epimorfismo $\varphi_{1}$. Portanto, existe apenas uma involução livre sobre $T^{4}$, a qual denotamos por $\tau_{3}$, tal que $T^{4} / \tau_{3}=\mathcal{M}_{N}^{1}$. 
(II) O segundo grupo não orientável é tal que

$$
\pi \cong \mathbb{Z} \times B_{2}=\left\langle t, x, y, z \mid[t, x], t y t^{-1}=y z,[z, t]^{\prime},[x, y],[x, z],[y, z]\right\rangle
$$

onde $B_{2}$ é um grupo de 3-variedade "flat" não orientável ([18]). Também podemos ver $\pi$ como o produto semidireto, $\mathbb{Z}^{3} \rtimes_{T} \mathbb{Z}$, onde a ação é determinada pela matriz

$$
T=\left(\begin{array}{ccc}
1 & 0 & 0 \\
0 & 1 & 0 \\
0 & 1 & -1
\end{array}\right)
$$

Denotemos por $\mathcal{M}_{N}^{2}$ a 4-variedade "flat" não orientável tal que $\pi_{1}\left(\mathcal{M}_{N}^{2}\right)=\pi$. Pela relação $t y t^{-1}=y z$, os epimorfismos de $\pi_{1}\left(\mathcal{M}_{N}^{2}\right)$ em $\mathbb{Z}_{2}$ são como os da Tabela 5.1, e uma apresentação de $\operatorname{Ker}\left(\varphi_{i}\right)$, para cada $i=1, \ldots, 7$, é dada na sequência.

- $\operatorname{Ker}\left(\varphi_{1}\right)=\left\langle y_{1}, y_{2}, y_{3}, y_{4}\right| y_{1}, y_{2}, y_{3}, y_{4}$ comutam $\rangle \cong \mathbb{Z}^{4}$, onde $y_{1}=t^{2}, y_{2}=x, y_{3}=y$ e $y_{4}=z$.

- $\operatorname{Ker}\left(\varphi_{2}\right)=\left\langle y_{1}, y_{2}, y_{3}, y_{4} \mid\left[y_{1}, y_{2}\right], y_{1} y_{3} y_{1}^{-1}=y_{3} y_{4},\left[y_{4}, y_{1}\right]^{\prime},\left[y_{2}, y_{3}\right],\left[y_{2}, y_{4}\right],\left[y_{3}, y_{4}\right]\right\rangle$, onde $y_{1}=t, y_{2}=x^{2}, y_{3}=y$ e $y_{4}=z$.

- $\operatorname{Ker}\left(\varphi_{3}\right)=\left\langle y_{1}, y_{2}, y_{3}, y_{4} \mid\left[y_{1}, y_{2}\right],\left[y_{3}, y_{1}\right] y_{4}^{2},\left[y_{4}, y_{1}\right]^{\prime},\left[y_{2}, y_{3}\right],\left[y_{2}, y_{4}\right],\left[y_{3}, y_{4}\right]\right\rangle$, onde $y_{1}=t$, $y_{2}=x, y_{3}=y^{2}$ e $y_{4}=z$.

- $\operatorname{Ker}\left(\varphi_{4}\right)=\left\langle y_{1}, y_{2}, y_{3}, y_{4} \mid\left[y_{1}, y_{2}\right],\left[y_{1}, y_{3}\right],\left[y_{1}, y_{4}\right],\left[y_{3}, y_{2}\right] y_{4},\left[y_{4}, y_{2}\right]^{\prime},\left[y_{3}, y_{4}\right]\right\rangle$, onde $y_{1}=t$, $y_{2}=x t^{-1}, y_{3}=y$ e $y_{4}=z$.

- $\operatorname{Ker}\left(\varphi_{5}\right)=\left\langle y_{1}, y_{2}, y_{3}, y_{4} \mid\left[y_{1}, y_{2}\right],\left[y_{3}, y_{1}\right] y_{4}^{2},\left[y_{4}, y_{1}\right]^{\prime},\left[y_{2}, y_{3}\right],\left[y_{2}, y_{4}\right],\left[y_{3}, y_{4}\right]\right\rangle$, onde $y_{1}=$ $t y^{-1}, y_{2}=x, y_{3}=y^{2}$ e $y_{4}=z$.

- $\operatorname{Ker}\left(\varphi_{6}\right)=\left\langle y_{1}, y_{2}, y_{3}, y_{4} \mid\left[y_{1}, y_{2}\right],\left[y_{3}, y_{1}\right] y_{4},\left[y_{4}, y_{1}\right]^{\prime},\left[y_{2}, y_{3}\right],\left[y_{2}, y_{4}\right],\left[y_{3}, y_{4}\right]\right\rangle$, onde $y_{1}=t$, $y_{2}=x^{2}, y_{3}=y x^{-1}$ e $y_{4}=z$.

- $\operatorname{Ker}\left(\varphi_{7}\right)=\left\langle y_{1}, y_{2}, y_{3}, y_{4} \mid\left[y_{1}, y_{2}\right] y_{4},\left[y_{3}, y_{1}\right] y_{4}^{2},\left[y_{4}, y_{1}\right]^{\prime},\left[y_{2}, y_{3}\right],\left[y_{2}, y_{4}\right],\left[y_{3}, y_{4}\right]\right\rangle$, onde $y_{1}=$ $t y^{-1}, y_{2}=x y^{-1}, y_{3}=y^{2}$ e $y_{4}=z$.

Analogamente aos casos anteriores, existe apenas uma involução livre sobre $T^{4}$, que denotamos por $\tau_{4}$, tal que $T^{4} / \tau_{4}=\mathcal{M}_{N}^{2}$, que está associada ao epimorfismo $\varphi_{1}$ (Tabela 5.1 .

(III) Finalmente, o terceiro grupo não orientável é tal que

$$
\pi \cong \mathbb{Z}^{3} \rtimes_{T} \mathbb{Z}
$$


onde a ação é dada pela matriz

$$
T=\left(\begin{array}{ccc}
-1 & 0 & 0 \\
0 & -1 & 0 \\
0 & 0 & -1
\end{array}\right)
$$

Assim,

$$
\pi=\left\langle t, x, y, z \mid[x, t]^{\prime},[y, t]^{\prime},[z, t]^{\prime},[x, y],[x, z],[y, z]\right\rangle .
$$

Denotemos por $\mathcal{M}_{N}^{3}$ a 4-variedade "flat" não orientável tal que $\pi_{1}\left(\mathcal{M}_{N}^{3}\right)=\pi$. São quinze os epimorfismos de $\pi_{1}\left(\mathcal{M}_{N}^{3}\right)$ em $\mathbb{Z}_{2}$, e eles são como os da Tabela 5.2. Assim, pelo método de Reidemeister-Schreier, obtemos:

- $\operatorname{Ker}\left(\varphi_{1}\right)=\left\langle y_{1}, y_{2}, y_{3}, y_{4}\right| y_{1}, y_{2}, y_{3}, y_{4}$ comutam $\rangle \cong \mathbb{Z}^{4}$, onde $y_{1}=t^{2}, y_{2}=x, y_{3}=y$, $y_{4}=z$.

- $\operatorname{Ker}\left(\varphi_{2}\right)=\left\langle y_{1}, y_{2}, y_{3}, y_{4} \mid\left[y_{2}, y_{1}\right]^{\prime},\left[y_{3}, y_{1}\right]^{\prime},\left[y_{4}, y_{1}\right]^{\prime},\left[y_{2}, y_{3}\right],\left[y_{2}, y_{4}\right],\left[y_{3}, y_{4}\right]\right\rangle$, onde $y_{1}=t$, $y_{2}=x^{2}, y_{3}=y, y_{4}=z$.

- $\operatorname{Ker}\left(\varphi_{3}\right)=\left\langle y_{1}, y_{2}, y_{3}, y_{4} \mid\left[y_{2}, y_{1}\right]^{\prime},\left[y_{3}, y_{1}\right]^{\prime},\left[y_{4}, y_{1}\right]^{\prime},\left[y_{2}, y_{3}\right],\left[y_{2}, y_{4}\right],\left[y_{3}, y_{4}\right]\right\rangle$, onde $y_{1}=t$, $y_{2}=x, y_{3}=y^{2}, y_{4}=z$.

- $\operatorname{Ker}\left(\varphi_{4}\right)=\left\langle y_{1}, y_{2}, y_{3}, y_{4} \mid\left[y_{2}, y_{1}\right]^{\prime},\left[y_{3}, y_{1}\right]^{\prime},\left[y_{4}, y_{1}\right]^{\prime},\left[y_{2}, y_{3}\right],\left[y_{2}, y_{4}\right],\left[y_{3}, y_{4}\right]\right\rangle$, onde $y_{1}=t$, $y_{2}=x, y_{3}=y, y_{4}=z^{2}$.

- $\operatorname{Ker}\left(\varphi_{5}\right)=\left\langle y_{1}, y_{2}, y_{3}, y_{4} \mid\left[y_{2}, y_{1}\right]^{\prime},\left[y_{3}, y_{1}\right]^{\prime},\left[y_{4}, y_{1}\right]^{\prime},\left[y_{2}, y_{3}\right],\left[y_{2}, y_{4}\right],\left[y_{3}, y_{4}\right]\right\rangle$, onde $y_{1}=t x^{-1}$, $y_{2}=x^{2}, y_{3}=y, y_{4}=z$.

- $\operatorname{Ker}\left(\varphi_{6}\right)=\left\langle y_{1}, y_{2}, y_{3}, y_{4} \mid\left[y_{2}, y_{1}\right]^{\prime},\left[y_{3}, y_{1}\right]^{\prime},\left[y_{4}, y_{1}\right]^{\prime},\left[y_{2}, y_{3}\right],\left[y_{2}, y_{4}\right],\left[y_{3}, y_{4}\right]\right\rangle$, onde $y_{1}=t y^{-1}$, $y_{2}=x, y_{3}=y^{2}, y_{4}=z$.

- $\operatorname{Ker}\left(\varphi_{7}\right)=\left\langle y_{1}, y_{2}, y_{3}, y_{4} \mid\left[y_{2}, y_{1}\right]^{\prime},\left[y_{3}, y_{1}\right]^{\prime},\left[y_{4}, y_{1}\right]^{\prime},\left[y_{2}, y_{3}\right],\left[y_{2}, y_{4}\right],\left[y_{3}, y_{4}\right]\right\rangle$, onde $y_{1}=t z^{-1}$, $y_{2}=x, y_{3}=y, y_{4}=z^{2}$.

- $\operatorname{Ker}\left(\varphi_{8}\right)=\left\langle y_{1}, y_{2}, y_{3}, y_{4} \mid\left[y_{2}, y_{1}\right]^{\prime},\left[y_{3}, y_{1}\right]^{\prime},\left[y_{4}, y_{1}\right]^{\prime},\left[y_{2}, y_{3}\right],\left[y_{2}, y_{4}\right],\left[y_{3}, y_{4}\right]\right\rangle$, onde $y_{1}=t$, $y_{2}=x^{2}, y_{3}=y x^{-1}, y_{4}=z$.

- $\operatorname{Ker}\left(\varphi_{9}\right)=\left\langle y_{1}, y_{2}, y_{3}, y_{4} \mid\left[y_{2}, y_{1}\right]^{\prime},\left[y_{3}, y_{1}\right]^{\prime},\left[y_{4}, y_{1}\right]^{\prime},\left[y_{2}, y_{3}\right],\left[y_{2}, y_{4}\right],\left[y_{3}, y_{4}\right]\right\rangle$, onde $y_{1}=t$, $y_{2}=x^{2}, y_{3}=y, y_{4}=z x^{-1}$.

- $\operatorname{Ker}\left(\varphi_{10}\right)=\left\langle y_{1}, y_{2}, y_{3}, y_{4} \mid\left[y_{2}, y_{1}\right]^{\prime},\left[y_{3}, y_{1}\right]^{\prime},\left[y_{4}, y_{1}\right]^{\prime},\left[y_{2}, y_{3}\right],\left[y_{2}, y_{4}\right],\left[y_{3}, y_{4}\right]\right\rangle$, onde $y_{1}=t$, $y_{2}=x, y_{3}=y^{2}, y_{4}=z y^{-1}$.

- $\operatorname{Ker}\left(\varphi_{11}\right)=\left\langle y_{1}, y_{2}, y_{3}, y_{4} \mid\left[y_{2}, y_{1}\right]^{\prime},\left[y_{3}, y_{1}\right]^{\prime},\left[y_{4}, y_{1}\right]^{\prime},\left[y_{2}, y_{3}\right],\left[y_{2}, y_{4}\right],\left[y_{3}, y_{4}\right]\right\rangle$, onde $y_{1}=t y^{-1}$, $y_{2}=x y^{-1}, y_{3}=y^{2}, y_{4}=z$.

- $\operatorname{Ker}\left(\varphi_{12}\right)=\left\langle y_{1}, y_{2}, y_{3}, y_{4} \mid\left[y_{2}, y_{1}\right]^{\prime},\left[y_{3}, y_{1}\right]^{\prime},\left[y_{4}, y_{1}\right]^{\prime},\left[y_{2}, y_{3}\right],\left[y_{2}, y_{4}\right],\left[y_{3}, y_{4}\right]\right\rangle$, onde $y_{1}=t x^{-1}$, $y_{2}=x^{2}, y_{3}=y, y_{4}=z x^{-1}$. 
- $\operatorname{Ker}\left(\varphi_{13}\right)=\left\langle y_{1}, y_{2}, y_{3}, y_{4} \mid\left[y_{2}, y_{1}\right]^{\prime},\left[y_{3}, y_{1}\right]^{\prime},\left[y_{4}, y_{1}\right]^{\prime},\left[y_{2}, y_{3}\right],\left[y_{2}, y_{4}\right],\left[y_{3}, y_{4}\right]\right\rangle$, onde $y_{1}=t y^{-1}$, $y_{2}=x, y_{3}=y^{2}, y_{4}=z y^{-1}$.

- $\operatorname{Ker}\left(\varphi_{14}\right)=\left\langle y_{1}, y_{2}, y_{3}, y_{4} \mid\left[y_{2}, y_{1}\right]^{\prime},\left[y_{3}, y_{1}\right]^{\prime},\left[y_{4}, y_{1}\right]^{\prime},\left[y_{2}, y_{3}\right],\left[y_{2}, y_{4}\right],\left[y_{3}, y_{4}\right]\right\rangle$, onde $y_{1}=t$, $y_{2}=x^{2}, y_{3}=y x^{-1}, y_{4}=z x^{-1}$.

- $\operatorname{Ker}\left(\varphi_{15}\right)=\left\langle y_{1}, y_{2}, y_{3}, y_{4} \mid\left[y_{2}, y_{1}\right]^{\prime},\left[y_{3}, y_{1}\right]^{\prime},\left[y_{4}, y_{1}\right]^{\prime},\left[y_{2}, y_{3}\right],\left[y_{2}, y_{4}\right],\left[y_{3}, y_{4}\right]\right\rangle$, onde $y_{1}=t y^{-1}$, $y_{2}=x y^{-1}, y_{3}=y^{2}, y_{4}=z y^{-1}$.

Neste caso também existe apenas uma involução livre sobre $T^{4}$, que denotamos por $\tau_{5}$, tal que $T^{4} / \tau_{5}=\mathcal{M}_{N}^{3}$, que está associado ao epimorfismo $\varphi_{1}$ (Tabela 5.2 .

\subsection{PBU para $\left(T^{4}, \tau ; \mathbb{R}^{n}\right)$}

Inicialmente estudaremos a propriedade de Borsuk-Ulam para $\left(T^{4}, \tau_{i} ; \mathbb{R}^{n}\right)$, com $i=1, \ldots$, 5 , conforme as notações apresentadas nas seções anteriores. Lembremos que os casos a serem analisados são $n=2,3,4$.

Proposição 5.2. A propriedade de Borsuk-Ulam não vale para $\left(T^{4}, \tau_{i} ; \mathbb{R}^{n}\right)$, para todo $i=1, \ldots, 5$ e $n=2,3,4$.

Demonstração. Das apresentações dos grupos de 4-variedade "flat", dadas anteriormente, podemos definir homomorfismo $\eta$ de $\pi_{1}\left(T^{4} / \tau_{i}\right)$ em $\mathbb{Z}$, para todo $i=1, \ldots, 5$, de modo que o diagrama $(*)$, do Teorema 1.20 , se fatore. Assim, a propriedade de Borsuk-Ulam não vale para $\left(T^{4}, \tau_{i} ; \mathbb{R}^{2}\right)$ para todo $i=1, \ldots, 5$. Consequentemente, do Lema $1.18(1)$, a propriedade de Borsuk-Ulam também não vale para $\left(T^{4}, \tau_{i} ; \mathbb{R}^{3}\right)$ e $\left(T^{4}, \tau_{i} ; \mathbb{R}^{4}\right)$, para todo $i=1, \ldots, 5$.

Como comentamos na Observação 5.1, existem involuções livres sobre $T^{4}$ tais que a variedade quociente também é $T^{4}$. Assim, na sequência, estudaremos a propriedade de Borsuk-Ulam para $\left(T^{4}, \tau ; \mathbb{R}^{n}\right)$ tal que $T^{4} / \tau=T^{4}$.

Proposição 5.3. Sejam $\tau$ e $\tau^{\prime}$ involuções livres sobre $T^{4}$ tais que $T^{4} / \tau=T^{4} / \tau^{\prime}=$ $T^{4}$. A propriedade de Borsuk-Ulam vale para $\left(T^{4}, \tau ; \mathbb{R}^{n}\right)$ se, e somente se, ela vale para $\left(T^{4}, \tau^{\prime} ; \mathbb{R}^{n}\right)$.

Demonstração. Sejam $\varphi$ e $\varphi^{\prime}$ os epimorfismos de $\pi_{1}\left(T^{4}\right)$ em $\mathbb{Z}_{2}$ associados aos revestimentos duplos $T^{4} \rightarrow T^{4} / \tau$ e $T^{4} \rightarrow T^{4} / \tau^{\prime}$, respectivamente. Como existe isomorfismo $\theta: \pi_{1}\left(T^{4} / \tau^{\prime}\right) \rightarrow \pi_{1}\left(T^{4} / \tau\right)\left(\left[12\right.\right.$, Proposição 30]) tal que $\varphi^{\prime}=\varphi \circ \theta$, e $T^{4} / \tau, T^{4} / \tau^{\prime}$ são 
$K(\pi, 1)$, existe equivalência de homotopia $f: T^{4} / \tau^{\prime} \rightarrow T^{4} / \tau([35$, Capítulo V, Seção 4]) tal que $f_{\#}=\theta$. Portanto, $f^{*}([\varphi])=\left[\varphi^{\prime}\right]$, e o resultado segue do Corolário 1.17 .

Daí:

Proposição 5.4. A propriedade de Borsuk-Ulam não vale para $\left(T^{4}, \tau ; \mathbb{R}^{n}\right)$ para todo $n \geq 2$ e para toda involução livre $\tau$ sobre $T^{4}$ tal que $T^{4} / \tau=T^{4}$.

Demonstração. Como vimos, na Observação 5.1, a involução diagonal livre $\tau \times i d$ sobre $T^{4}=T^{2} \times T^{2}$ é tal que $T^{4} /(\tau \times i d)=T^{4}$. Como, pelo Teorema 2.1(a), a propriedade de Borsuk-Ulam não vale para $\left(T^{2}, \tau ; \mathbb{R}^{2}\right)$, e consequentemente, pelo Lema $1.18(1)$, ela também não vale para $\left(T^{2}, \tau ; \mathbb{R}^{n}\right)$ quando $n=3,4$, da Proposição 2.3 , segue que a propriedade de Borsuk-Ulam não vale para $\left(T^{4}, \tau \times i d ; \mathbb{R}^{n}\right)$ quando $n=2,3,4$. Daí, da proposição anterior, temos o resultado.

Assim, do que foi feito neste capítulo, a conclusão que tiramos é que a propriedade de Borsuk-Ulam não vale para $\left(T^{4}, \tau ; \mathbb{R}^{n}\right)$ para todo $n \geq 2$, e para toda involução livre $\tau$ sobre $T^{4}$. 


\section{Capítulo 6}

\section{PBU para revestimentos duplos de $\frac{K b \times K b}{\tau \times \beta}$}

Neste capítulo, apresentaremos mais exemplos de elementos da subfamília $\mathcal{F}_{3}$ equipados com involuções livres, e estudaremos a propriedade de Borsuk-Ulam para os mesmos.

\subsection{Revestimentos duplos de $\frac{K b \times K b}{\tau \times \beta}$}

Consideremos o produto $K b \times K b$ equipado com a involução livre da forma diagonal $\tau_{1}:=\tau \times \beta$ de modo que $\tau$ e $\beta$ são involuções livres sobre $K b$ que correspondem à sequência $(\overline{1}, \overline{0})$, conforme a caracterização dada em [11]. Na prova do Teorema 2.8, para o caso particular $M_{1}=M_{2}=K b$, a 4 -variedade quociente

$$
\frac{K b \times K b}{\tau_{1}}
$$

é tal que $\pi_{1}\left((K b \times K b) / \tau_{1}\right)=\operatorname{Ker}(\bar{\varphi})$, onde $\bar{\varphi}=\omega \circ(\psi, \phi)$ é um epimorfismo de

$$
\begin{aligned}
\pi_{1}(K b / \tau \times K b / \beta) & =\left\langle u, v \mid[u, v]^{\prime}\right\rangle \times\left\langle\widetilde{u}, \widetilde{v} \mid[\widetilde{u}, \widetilde{v}]^{\prime}\right\rangle \\
& =\left\langle u, v, \widetilde{u}, \widetilde{v} \mid[u, v]^{\prime},[\widetilde{u}, \widetilde{v}]^{\prime},[u, \widetilde{u}],[u, \widetilde{v}],[v, \widetilde{u}],[v, \widetilde{v}]\right\rangle
\end{aligned}
$$

em $\mathbb{Z}_{2}$, com $\omega: \mathbb{Z}_{2} \oplus \mathbb{Z}_{2} \rightarrow \mathbb{Z}_{2}$ dado por $\omega(\bar{a}, \bar{b})=\bar{a}+\bar{b}, \psi: \pi_{1}\left(W_{1}\right) \rightarrow \mathbb{Z}_{2}$ e $\phi: \pi_{1}\left(W_{2}\right) \rightarrow \mathbb{Z}_{2}$ tais que $\psi(u)=\phi(\widetilde{u})=\overline{1}$ e $\psi(v)=\phi(\widetilde{v})=\overline{0}$. Assim, $\bar{\varphi}$ envia $u, \widetilde{u}$ em $\overline{1}$, e $v, \widetilde{v}$ em $\overline{0}$. Daí, seja $\{1, u\}$ um sistema de Schreier. Pelo método de Reidemeister-Schreier, temos os seguintes geradores para $\pi_{1}\left((K b \times K b) / \tau_{1}\right)$ :

$y_{12}=u^{2}, y_{21}=v, y_{22}=u v u^{-1}, y_{31}=\widetilde{u} u^{-1}, y_{32}=u \widetilde{u}, y_{41}=\widetilde{v}$ e $y_{42}=u \widetilde{v} u^{-1}$,

e as seguintes relações: 
(1) $y_{22} y_{12} y_{21}^{-1}=1$

(2) $y_{12} y_{21} y_{22}^{-1}=1$

(3) $y_{31} y_{42} y_{32} y_{41}^{-1}=1$

(4) $y_{32} y_{41} y_{31} y_{42}^{-1}=1$

(5) $y_{32} y_{12}^{-1} y_{31}^{-1}=1$

(6) $y_{12} y_{31} y_{32}^{-1}=1$

(7) $y_{42} y_{41}^{-1}=1$

(8) $y_{12} y_{41} y_{12}^{-1} y_{42}^{-1}=1$

(9) $y_{21} y_{31} y_{22}^{-1} y_{31}^{-1}=1$

(10) $y_{22} y_{32} y_{21}^{-1} y_{32}^{-1}=1$

(11) $y_{21} y_{41} y_{21}^{-1} y_{41}^{-1}=1$

(12) $y_{22} y_{42} y_{22}^{-1} y_{42}^{-1}=1$.

Com a notação $x=y_{12}, y=y_{21}, z=y_{31}$ e $w=y_{41}$, temos a seguinte apresentação:

$$
\pi_{1}\left((K b \times K b) / \tau_{1}\right)=\left\langle x, y, z, w \mid[x, y]^{\prime},[x, z],[x, w],[y, w],[z, y] x,[z, w]^{\prime} x\right\rangle .
$$

Lema 6.1. De acordo com a notação anterior, a 4 -variedade $(K b \times K b) / \tau_{1}$ é homotopicamente equivalente ao espaço total de um $T^{2}$-fibrado sobre $T^{2}$.

Demonstração. De (6.1), o subgrupo gerado por $\{x, z\},\langle x, z\rangle$, é um subgrupo normal de $\pi_{1}\left((K b \times K b) / \tau_{1}\right) \mathrm{e}$

$$
\frac{\pi_{1}\left((K b \times K b) / \tau_{1}\right)}{\langle x, z\rangle}=\langle\bar{y}, \bar{w} \mid[\bar{y}, \bar{w}]\rangle \cong \pi_{1}\left(T^{2}\right) .
$$

Logo, temos a seguinte sequência exata

$$
1 \longrightarrow\langle x, z\rangle \longrightarrow \pi_{1}\left((K b \times K b) / \tau_{1}\right) \longrightarrow \pi_{1}\left(T^{2}\right) \longrightarrow 1
$$

que cinde, ou seja, $\pi_{1}\left((K b \times K b) / \tau_{1}\right)=\langle x, z\rangle \rtimes_{\theta} \mathbb{Z}^{2}$, onde $\theta: \mathbb{Z}^{2} \rightarrow \operatorname{Aut}(\langle x, z\rangle)$ é tal que

- $\theta_{\bar{y}}(x)=x^{-1}$

- $\theta_{\bar{y}}(z)=x z$
- $\theta_{\bar{w}}(x)=x$

- $\theta_{\bar{w}}(z)=z^{-1} x^{-1}$.

Observemos que $\langle x, z\rangle \cong \pi_{1}\left(T^{2}\right)$. De fato, consideremos o produto semidireto

$$
\mathbb{Z}^{2} \rtimes_{\theta^{\prime}} \mathbb{Z}^{2}=\langle a, b \mid[a, b]\rangle \rtimes_{\theta^{\prime}}\langle c, d \mid[c, d]\rangle,
$$

onde $\theta^{\prime}:\langle c, d \mid[c, d]\rangle \rightarrow \operatorname{Aut}(\langle a, b \mid[a, b]\rangle)$ é dado por $\theta_{c}^{\prime}(a)=a^{-1}, \theta_{c}^{\prime}(b)=a b, \theta_{d}^{\prime}(a)=a$, $\theta_{d}^{\prime}(b)=b^{-1} a^{-1}$. Temos $\theta_{c}^{\prime} \circ \theta_{d}^{\prime}=\theta_{d}^{\prime} \circ \theta_{c}^{\prime}$, e $\theta_{c}^{\prime}([a, b])=\theta_{d}^{\prime}([a, b])=1$. Assim, por [21, Cap. 
10, Corolário 1], uma apresentação de $\mathbb{Z}^{2} \rtimes_{\theta^{\prime}} \mathbb{Z}^{2}$ é dada da seguinte forma:

geradores: $a, b, c, d$

\section{relações:}

(1) $[a, b]=1$,

(2) $[c, d]=1$,

(3) $c a c^{-1} \theta_{c}^{\prime}(a)^{-1}=1 \Rightarrow[a, c]^{\prime}=1$,

(4) $c b c^{-1} \theta_{c}^{\prime}(b)^{-1}=1 \Rightarrow[c, b] a^{-1}=1$,

(5) $d_{a d} d^{-1} \theta_{d}^{\prime}(a)^{-1}=1 \Rightarrow[a, d]=1$

(6) $d b d^{-1} \theta_{d}^{\prime}(b)^{-1}=1 \Rightarrow[b, d]^{\prime} a=1$,

ou seja, $\pi_{1}\left((K b \times K b) / \tau_{1}\right) \stackrel{\zeta}{\cong} \mathbb{Z}^{2} \rtimes_{\theta^{\prime}} \mathbb{Z}^{2}$, onde $\zeta$ é dado por $\zeta(x)=a, \zeta(y)=c, \zeta(z)=b$ e $\zeta(w)=d$. Logo, $\pi_{1}\left((K b \times K b) / \tau_{1}\right)$ é uma extensão de $\pi_{1}\left(T^{2}\right)$ por $\pi_{1}\left(T^{2}\right)$. Como

$$
\chi\left((K b \times K b) / \tau_{1}\right)=\frac{\chi(K b) \chi(K b)}{2}=0=\chi\left(T^{2}\right) \chi\left(T^{2}\right),
$$

por [19, Teorema 5.2], segue que $(K b \times K b) / \tau_{1}$ é homotopicamente equivalente ao espaço total de um $T^{2}$-fibrado sobre $T^{2}$.

Agora, seja $\varphi$ um epimorfismo de $\pi_{1}\left((K b \times K b) / \tau_{1}\right)$ em $\mathbb{Z}_{2}$. Da relação $[z, y] x=1$, de (6.1), segue que $\varphi(x)=\overline{0}$. Daí, temos sete possibilidades para $\varphi$, conforme a seguinte tabela:

\begin{tabular}{|l|c|c|c|c|}
\hline & $\boldsymbol{x}$ & $\boldsymbol{y}$ & $\boldsymbol{z}$ & $\boldsymbol{w}$ \\
\hline $\boldsymbol{\varphi}_{\mathbf{1}}$ & $\overline{0}$ & $\overline{1}$ & $\overline{0}$ & $\overline{0}$ \\
\hline $\boldsymbol{\varphi}_{\mathbf{2}}$ & $\overline{0}$ & $\overline{0}$ & $\overline{1}$ & $\overline{0}$ \\
\hline $\boldsymbol{\varphi}_{\mathbf{3}}$ & $\overline{0}$ & $\overline{0}$ & $\overline{0}$ & $\overline{1}$ \\
\hline $\boldsymbol{\varphi}_{\mathbf{4}}$ & $\overline{0}$ & $\overline{1}$ & $\overline{1}$ & $\overline{0}$ \\
\hline
\end{tabular}

\begin{tabular}{|l|l|l|l|l|}
\hline & $\boldsymbol{x}$ & $\boldsymbol{y}$ & $\boldsymbol{z}$ & $\boldsymbol{w}$ \\
\hline $\boldsymbol{\varphi}_{\mathbf{5}}$ & $\overline{0}$ & $\overline{1}$ & $\overline{0}$ & $\overline{1}$ \\
\hline $\boldsymbol{\varphi}_{\mathbf{6}}$ & $\overline{0}$ & $\overline{0}$ & $\overline{1}$ & $\overline{1}$ \\
\hline $\boldsymbol{\varphi}_{\mathbf{7}}$ & $\overline{0}$ & $\overline{1}$ & $\overline{1}$ & $\overline{1}$ \\
\hline
\end{tabular}

Tabela 6.1: Epimorfismos de $\pi_{1}\left((K b \times K b) / \tau_{1}\right)$ em $\mathbb{Z}_{2}$.

Denotemos por $W_{i}$ o revestimento duplo de $(K b \times K b) / \tau_{1}$ associado a $\varphi_{i}\left(\pi_{1}\left(W_{i}\right)=\right.$ $\left.\operatorname{Ker}\left(\varphi_{i}\right)\right)$, e por $\tau_{\varphi_{i}}$ a involução livre sobre $W_{i}$ (dada pela "deck transformation"), $i=$ $1, \ldots, 7$. Pelos Teorema 3.5 e Lema 3.4. $W_{i} \in \mathcal{F}_{3}$ e tem o mesmo tipo de homotopia do espaço total de um $T^{2}$-fibrado sobre $T^{2}$, para cada $i=1, \ldots, 7$. Assim, quanto a propriedade de Borsuk-Ulam para $\left(W_{i}, \tau_{\varphi_{i}} ; \mathbb{R}^{n}\right), i=1, \ldots, 7$, obtemos:

Proposição 6.2. De acordo com as notações anteriores, a propriedade de Borsuk-Ulam vale para $\left(W_{i}, \tau_{\varphi_{i}} ; \mathbb{R}^{2}\right)$ se, e somente se, $i=2,4,6,7$. 
Demonstração. Suponhamos que o diagrama $(*)$, do Teorema 1.20, se fatore. Pelas relações $[z, y] x=1 \mathrm{e}[z, w]^{\prime} x=1$, de $(6.1)$, temos $\eta(x)=\eta(z)=0$. Então, para $i=2,4,6,7$, temos uma contradição, pois nestes casos $\eta(z)$ deve ser um inteiro ímpar, uma vez que $\varphi_{i}(z)=\overline{1}$. Portanto, a propriedade de Borsuk-Ulam vale para $\left(W_{i}, \tau_{\varphi_{i}} ; \mathbb{R}^{2}\right)$ para $i=2,4,6,7$. Agora, para $i=1,3,5$, podemos definir um homomorfismo $\eta$ de modo que o diagrama $(*)$ se fatore, ou seja, para tais $i$ 's a propriedade de Borsuk-Ulam não vale $\operatorname{para}\left(W_{i}, \tau_{\varphi_{i}} ; \mathbb{R}^{2}\right)$.

Observação 6.3. Da Proposição 6.2 e do Lema 1.18(1), a propriedade de Borsuk-Ulam não vale para $\left(W_{i}, \tau_{\varphi_{i}} ; \mathbb{R}^{n}\right)$, se $i=1,3,5$ e $n=3,4$. Assim, resta-nos estudar a propriedade para $\left(W_{i}, \tau_{\varphi_{i}} ; \mathbb{R}^{n}\right)$, se $i=2,4,6,7$ e $n=3,4$.

Para $n=4$, temos:

Proposição 6.4. Com as notações anteriores, a propriedade de Borsuk-Ulam não vale para $\left(W_{i}, \tau_{\varphi_{i}} ; \mathbb{R}^{4}\right)$, quando $i=2,4,6,7$.

Demonstração. O subgrupo gerado por $\{x\},\langle x\rangle$, é normal em $\pi_{1}\left((K b \times K b) / \tau_{1}\right)$ (em (6.1)) e

$$
G=\frac{\pi_{1}\left((K b \times K b) / \tau_{1}\right)}{\langle x\rangle}=\left\langle\bar{y}, \bar{z}, \bar{w} \mid[\bar{y}, \bar{w}],[\bar{y}, \bar{z}],[\bar{z}, \bar{w}]^{\prime}\right\rangle,
$$

ou seja, temos a seguinte sequência exata curta

$$
1 \longrightarrow\langle x\rangle \longrightarrow \pi_{1}\left((K b \times K b) / \tau_{1}\right) \stackrel{\nu}{\longrightarrow} G \longrightarrow 1
$$

Agora, o subgrupo gerado por $\{\bar{y}, \bar{z}\}$ é normal em $G$ e

$$
\frac{G}{\langle\bar{y}, \bar{z}\rangle}=\langle t\rangle \cong \mathbb{Z}
$$

onde $t=\overline{\bar{w}}$. Logo, $G=\langle\bar{y}, \bar{z}\rangle \rtimes_{\theta} \mathbb{Z}$, onde $\theta: \mathbb{Z} \rightarrow \operatorname{Aut}(\langle\bar{y}, \bar{z}\rangle)$ é dado por

- $\theta_{t}(\bar{y})=\bar{y}$

- $\theta_{t}(\bar{z})=\bar{z}^{-1}$

Verifica-se que $G=\langle\bar{y}, \bar{z} \mid[\bar{y}, \bar{z}]\rangle \rtimes_{\theta} \mathbb{Z} \cong \mathbb{Z}^{2} \rtimes_{\theta} \mathbb{Z}$, onde o homomorfismo $\theta$ está associado à matriz

$$
\left(\begin{array}{cc}
1 & 0 \\
0 & -1
\end{array}\right) .
$$

Por [18], o grupo $G$ é o grupo de 3-variedade "flat" não orientável $B_{1}$, isto é, $G=\pi_{1}(\mathcal{X})$, onde $\mathcal{X}$ é uma 3 -variedade "flat" não orientável. Na verdade, $\mathcal{X} \simeq K b \times \mathbb{S}^{1}$, pois $\mathcal{X}$ e 
$K b \times \mathbb{S}^{1}$ são $K(\pi, 1)$ e $\pi_{1}\left(K b \times \mathbb{S}^{1}\right)=\left\langle a, b, c \mid[a, b]^{\prime},[a, c],[b, c]\right\rangle \cong G$. Assim, consideremos os seguintes epimorfismos de $\pi_{1}(\mathcal{X})$ em $\mathbb{Z}_{2}$ :

- $\bar{\varphi}_{2}(\bar{y})=\overline{0}, \bar{\varphi}_{2}(\bar{z})=\overline{1}, \bar{\varphi}_{2}(\bar{w})=\overline{0}$;

- $\bar{\varphi}_{4}(\bar{y})=\overline{1}, \bar{\varphi}_{4}(\bar{z})=\overline{1}, \bar{\varphi}_{4}(\bar{w})=\overline{0}$;

- $\bar{\varphi}_{6}(\bar{y})=\overline{0}, \bar{\varphi}_{6}(\bar{z})=\overline{1}, \bar{\varphi}_{6}(\bar{w})=\overline{1}$;

- $\bar{\varphi}_{7}(\bar{y})=\overline{1}, \bar{\varphi}_{7}(\bar{z})=\overline{1}, \bar{\varphi}_{7}(\bar{w})=\overline{1}$.

Denotemos por $\mathcal{X}_{i}$ o revestimento duplo de $\mathcal{X}$ associado a $\bar{\varphi}_{i}, i=2,4,6,7$. Pelo método de Reidemeister-Schreier, na sequência, damos uma apresentação para $\pi_{1}\left(\mathcal{X}_{i}\right)=\operatorname{Ker}\left(\bar{\varphi}_{i}\right)$, para cada $i=2,4,6,7$, e verificaremos a qual 3-variedade "flat" corresponde $\mathcal{X}_{i}$.

- $\pi_{1}\left(\mathcal{X}_{2}\right)=\left\langle x_{1}, x_{2}, x_{3} \mid\left[x_{1}, x_{2}\right],\left[x_{1}, x_{3}\right],\left[x_{2}, x_{3}\right]^{\prime}\right\rangle$, onde $x_{1}=\bar{y}, x_{2}=\bar{z}^{2}$ e $x_{3}=\bar{w}$. Temos $\left\langle x_{1}, x_{2}\right\rangle \triangleleft \pi_{1}\left(\mathcal{X}_{2}\right)$, com $\pi_{1}\left(\mathcal{X}_{2}\right) /\left\langle x_{1}, x_{2}\right\rangle \cong \mathbb{Z}$. Mostra-se que $\pi_{1}\left(\mathcal{X}_{2}\right)=\mathbb{Z}^{2} \rtimes_{\theta^{\prime}} \mathbb{Z}$ com $\theta^{\prime}$ associado à matriz

$$
\left(\begin{array}{cc}
1 & 0 \\
0 & -1
\end{array}\right) .
$$

Portanto, $\pi_{1}\left(\mathcal{X}_{2}\right)=B_{1}$, o grupo de 3 -variedade "flat" não orientável ([18]), ou seja, $\mathcal{X}_{2}=\mathcal{X}$.

- $\pi_{1}\left(\mathcal{X}_{6}\right)=\left\langle y_{1}, y_{2}, y_{3} \mid\left[y_{1}, y_{2}\right],\left[y_{1}, y_{3}\right],\left[y_{2}, y_{3}\right]^{\prime}\right\rangle \cong \pi_{1}\left(\mathcal{X}_{2}\right)$, onde $y_{1}=\bar{y}, y_{2}=\bar{z}^{2}$ e $y_{3}=$ $\bar{w} \cdot \bar{z}^{-1}$, isto é, $\mathcal{X}_{6}=\mathcal{X}$.

- $\pi_{1}\left(\mathcal{X}_{4}\right)=\left\langle z_{1}, z_{2}, z_{3} \mid\left[z_{1}, z_{2}\right],\left[z_{1}, z_{3}\right],\left[z_{2}, z_{3}\right]^{\prime} z_{1}\right\rangle$, onde $z_{1}=\bar{y}^{2}, z_{2}=\bar{z} \cdot \bar{y}^{-1}$ e $z_{3}=\bar{w}$. Agora, $\left\langle z_{1}, z_{2}\right\rangle \triangleleft \pi_{1}\left(\mathcal{X}_{4}\right)$, com $\frac{\pi_{1}\left(\mathcal{X}_{4}\right)}{\left\langle z_{1}, z_{2}\right\rangle} \cong \mathbb{Z}$. Logo, $\pi_{1}\left(\mathcal{X}_{4}\right)=\mathbb{Z}^{2} \rtimes_{\theta^{\prime}} \mathbb{Z}$ com $\theta^{\prime}$ associado à matriz

$$
\left(\begin{array}{ll}
1 & -1 \\
0 & -1
\end{array}\right)
$$

Tal matriz é equivalente a

$$
\left(\begin{array}{ll}
0 & 1 \\
1 & 0
\end{array}\right),
$$

conjugando-a por

$$
\left(\begin{array}{cc}
1 & 0 \\
1 & -1
\end{array}\right) .
$$

Daí, $\pi_{1}\left(\mathcal{X}_{4}\right)=B_{2}$, o grupo de 3-variedade "flat" não orientável ([18]).

- $\pi_{1}\left(\mathcal{X}_{7}\right)=\left\langle w_{1}, w_{2}, w_{3} \mid\left[w_{1}, w_{2}\right],\left[w_{1}, w_{3}\right],\left[w_{2}, w_{3}\right]^{\prime} w_{1}\right\rangle \cong \pi_{1}\left(\mathcal{X}_{4}\right)$, onde $w_{1}=\bar{y}^{2}, w_{2}=\bar{z} \cdot \bar{y}^{-1}$ e $w_{3}=\bar{w} \cdot \bar{y}^{-1}$, isto é, $\pi_{1}\left(\mathcal{X}_{7}\right)=B_{2}$.

Denotemos por $q_{i}: \mathcal{X}_{i} \rightarrow \mathcal{X}, i=2,4,6,7$, a projeção de revestimento duplo. Notemos 
que existe aplicação $f:(K b \times K b) / \tau_{1} \rightarrow \mathcal{X}$ tal que $f_{\#}=\nu$ ([35, Cap. V, Corolário 4.4]). Assim, consideremos a fibração induzida de $\left(\mathcal{X}_{i}, q_{i}\right)$ por $f$ conforme o seguinte diagrama

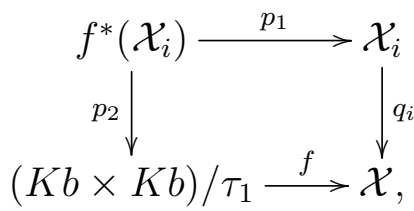

onde $f^{*}\left(\mathcal{X}_{i}\right)=\left\{(u, v) \in \mathcal{X}_{i} \times(K b \times K b) / \tau_{1} \mid q_{i}(u)=f(v)\right\}, i=2,4,6,7$, é revestimento duplo de $(K b \times K b) / \tau_{1}, p_{1}$ e $p_{2}$ são as projeções na primeira e segunda coordenadas, respectivamente. Temos $f^{*}\left(\mathcal{X}_{i}\right)=W_{i}$, uma vez que $\left(f^{*}\left(\mathcal{X}_{i}\right), p_{2}\right)$ é revestimento duplo de $(K b \times K b) / \tau_{1}$ e seu epimorfismo associado é $\varphi_{i}, i=2,4,6,7$. Além disso, se denotamos por $\beta_{i}$ a involução livre sobre $\mathcal{X}_{i}$ (dada pela "deck transformation"), a projeção $p_{1}:\left(W_{i}, \tau_{\varphi_{i}}\right) \rightarrow\left(\mathcal{X}_{i}, \beta_{i}\right)$ é equivariante. De fato, seja $x=(u, v) \in W_{i}$. Assim, $p_{1}(x)=u$, e como $\tau_{\varphi_{i}}(x) \neq x$ e $p_{2}\left(\tau_{\varphi_{i}}(x)\right)=p_{2}(x)=v$, temos

$$
\tau_{\varphi_{i}}(x)=\left(u^{\prime}, v\right), \text { com } u^{\prime} \neq u
$$

Agora, da comutatividade do diagrama anterior,

$$
q_{i}\left(p_{1}(x)\right)=q_{i}(u)=f(v)=q_{i}\left(p_{1}\left(\tau_{\varphi_{i}}(x)\right)\right),
$$

ou seja, $p_{1}(x), p_{1}\left(\tau_{\varphi_{i}}(x)\right) \in q_{i}^{-1}(f(v))$. Logo, por $(6.2), p_{1}\left(\tau_{\varphi_{i}}(x)\right)=\beta_{i}(u)$. Portanto,

$$
p_{1}\left(\tau_{\varphi_{i}}(x)\right)=\beta_{i}(u)=\beta_{i}\left(p_{1}(u, v)\right)=\beta_{i}\left(p_{1}(x)\right)
$$

para cada $i=2,4,6,7$. Uma vez que a propriedade de Borsuk-Ulam não vale para $\left(\mathcal{X}_{i}, \beta_{i} ; \mathbb{R}^{4}\right)$, pois $\operatorname{dim}\left(\mathcal{X}_{i}\right)=3$, obtemos aplicação equivariante de $\mathcal{X}_{i}$ em $\mathbb{S}^{3}$, que compondoa com $p_{1}$, obtemos aplicação equivariante de $W_{i}$ em $\mathbb{S}^{3}$. Logo, pela Proposição 1.16 (equivalência (i) e (ii)), a propriedade de Borsuk-Ulam não vale para $\left(W_{i}, \tau_{\varphi_{i}} ; \mathbb{R}^{4}\right), i=$ $2,4,6,7$.

O estudo da propriedade de Borsuk-Ulam para os revestimentos duplos de 3-variedades "flat" está sendo feito em [1], e pelos resultados lá obtidos, vem que:

Proposição 6.5. De acordo com as notações anteriores, a propriedade de Borsuk-Ulam não vale para $\left(W_{i}, \tau_{\varphi_{i}} ; \mathbb{R}^{3}\right)$ quando $i=2,6$.

Demonstração. Por [1], a propriedade de Borsuk-Ulam não vale para $\left(\mathcal{X}_{i}, \beta_{i} ; \mathbb{R}^{3}\right)$, quando $i=2,6$. Portanto, por argumento análogo ao usado no final da proposição anterior, da equivariância de $p_{1}: W_{i} \rightarrow \mathcal{X}_{i}$, o resultado segue da Proposição 1.16 (equivalência (i) e (ii)). 


\section{Observação 6.6.}

(1) Em [1], temos que a propriedade de Borsuk-Ulam vale para $\left(\mathcal{X}_{i}, \beta_{i} ; \mathbb{R}^{3}\right)$, quando $i=$ 4,7. Assim, por enquanto, não podemos concluir sobre a validade da propriedade para $\left(W_{i}, \tau_{\varphi_{i}} ; \mathbb{R}^{3}\right)$ quando $i=4,7$.

(2) Existem outras três involuções livres da forma $\tau \times \beta$ sobre $K b \times K b$ de modo que $\left(K b, \tau ; \mathbb{R}^{2}\right)$ e $\left(K b, \beta ; \mathbb{R}^{2}\right)$ satisfaçam a propriedade de Borsuk-Ulam, são elas: $\tau_{2}:=\tau \times \beta$, onde $\tau$ e $\beta$ correspondem à sequência $(\overline{1}, \overline{1}), \tau_{3}:=\tau \times \beta$, onde $\tau$ e $\beta$ correspondem às sequências $(\overline{1}, \overline{0})$ e $(\overline{1}, \overline{1})$, respectivamente, e $\tau_{4}:=\tau \times \beta$, onde $\tau$ e $\beta$ correspondem às sequências $(\overline{1}, \overline{1})$ e $(\overline{1}, \overline{0})$, respectivamente. De modo análogo ao que foi feito para a involução $\tau_{1}$, para $\tau_{2}$ temos

$$
\pi_{1}\left((K b \times K b) / \tau_{2}\right)=\left\langle x, y, z, w \mid[x, y]^{\prime},[x, z],[x, w],[z, y] x,[w, y] x,[z, w]^{\prime} x\right\rangle,
$$

para $\tau_{3}$ temos

$$
\pi_{1}\left((K b \times K b) / \tau_{3}\right)=\left\langle x, y, z, w \mid[x, y]^{\prime},[x, z],[x, w],[y, z] x^{-1},[y, w] x^{-1},[z, w]^{\prime} x\right\rangle
$$

e para $\tau_{4}$ temos

$$
\pi_{1}\left((K b \times K b) / \tau_{4}\right)=\left\langle x, y, z, w \mid[x, y]^{\prime},[x, z],[x, w],[y, w],[y, z] x^{-1},[z, w]^{\prime} x\right\rangle .
$$

Daí, como $\pi_{1}\left((K b \times K b) / \tau_{1}\right) \cong \pi_{1}\left((K b \times K b) / \tau_{4}\right)$, e $(K b \times K b) / \tau_{1}$ e $(K b \times K b) / \tau_{4}$ são $K(\pi, 1)$, segue que estas duas 4-variedades quocientes têm o mesmo tipo de homotopia. $\mathrm{O}$ mesmo acontece com as 4 -variedades quocientes $(K b \times K b) / \tau_{2}$ e $(K b \times K b) / \tau_{3}$. Agora, se consideramos um outro sistema de Schreier no cálculo da apresentação de $\pi_{1}((K b \times$ $K b) / \tau_{4}$ ), obtemos a seguinte apresentação

$$
\pi_{1}\left((K b \times K b) / \tau_{4}\right)=\left\langle x, y, z, w \mid[x, y]^{\prime} z,[z, w]^{\prime},[x, z],[x, w] z,[y, z],[y, w] z\right\rangle
$$

isto é, $\pi_{1}\left((K b \times K b) / \tau_{4}\right) \cong \pi_{1}\left((K b \times K b) / \tau_{2}\right)$, e portanto todas as quatro variedades quocientes têm o mesmo tipo de homotopia. Portanto, os revestimentos duplos de $(K b \times$ $K b) / \tau_{j}, j=2,3,4$, têm os mesmos tipos de homotopia dos revestimentos duplos de $(K b \times K b) / \tau_{1}$, ou seja, com estas outras involuções livres sobre $K b \times K b$ não obtemos "novos" elementos da subfamília $\mathcal{F}_{3}$ além dos obtidos usando $\tau_{1}$. 


\section{Capítulo 7}

\section{PBU para aplicações em variedades}

Sejam $X$ e $Y$ espaços, e $\tau$ uma involução livre sobre $X$. Neste capítulo, apresentaremos uma condição necessária e suficiente já conhecida, para a validade da propriedade de Borsuk-Ulam para a tripla $(X, \tau ; Y)$, em termos de existência de aplicação equivariante. Para o caso particular em que $X=\mathbb{S}^{n}$ e $Y=M^{k}$ é uma variedade de dimensão $k$, a princípio diferenciável, de [6, Teorema 33.1], faremos algumas observações à respeito da validade da propriedade de Borsuk-Ulam para $\left(\mathbb{S}^{n}, A ; M^{k}\right)$, e sob certas condições sobre $M^{k}$, calcularemos o conível (Definição 1.23) de $\left(M^{k}\right)^{*}=M^{k} \times M^{k}-\left\{(x, x) \mid x \in M^{k}\right\}$.

\subsection{Equivalência}

Seja $Y^{*}:=Y \times Y-\Delta$, onde $\Delta=\{(y, y) \mid y \in Y\}$ é a diagonal de $Y \times Y$, e seja $\alpha$ a involução livre sobre $Y^{*}$ que troca as coordenadas, isto é, $\alpha\left(y_{1}, y_{2}\right)=\left(y_{2}, y_{1}\right)$ para todo $\left(y_{1}, y_{2}\right) \in Y^{*}$.

Proposição 7.1. A propriedade de Borsuk-Ulam vale para $(X, \tau ; Y)$ se, e somente se, não existe aplicação equivariante de $(X, \tau)$ em $\left(Y^{*}, \alpha\right)$.

Demonstração. Suponhamos que exista aplicação equivariante $g: X \rightarrow Y^{*}$. Assim, consideremos a projeção na primeira coordenada $p_{1}: Y^{*} \rightarrow Y$. Logo, a aplicação $p_{1} \circ g$ : $X \rightarrow Y$ é contínua e tal que $\left(p_{1} \circ g\right)(x) \neq\left(p_{1} \circ g\right)(\tau(x))$, para todo $x \in X$, pois se $g(x)=\left(y_{1}, y_{2}\right) \in Y^{*}$, então $p_{1}(g(x))=p_{1}\left(y_{1}, y_{2}\right)=y_{1} \neq y_{2}=p_{1}\left(y_{2}, y_{1}\right)=p_{1}\left(\alpha\left(y_{1}, y_{2}\right)\right)=p_{1}(\alpha(g(x)))=p_{1}(g(\tau(x)))$.

Portanto, a propriedade de Borsuk-Ulam não vale para $(X, \tau ; Y)$. Reciprocamente, suponhamos que a propriedade de Borsuk-Ulam não valha para $(X, \tau ; Y)$. Então, existe 
aplicação $f: X \rightarrow Y$ tal que $f(x) \neq f(\tau(x))$, para todo $x \in X$. Desta forma, a aplicação $g: X \rightarrow Y^{*}$ definida por $g(x)=(f(x), f \circ \tau(x))$, é equivariante com respeito às involuções livres $\tau$ e $\alpha$ sobre $X$ e $Y^{*}$, respectivamente. Com efeito,

$$
g(\tau(x))=\left(f(\tau(x)), f\left(\tau^{2}(x)\right)\right)=(f(\tau(x)), f(x))=\alpha(f(x), f \circ \tau(x))=\alpha(g(x)) .
$$

E isto conclui a prova.

Observação 7.2. Pela proposição anterior, quando $X=\mathbb{S}^{n}$, estudar a propriedade de Borsuk-Ulam para ternas do tipo $\left(\mathbb{S}^{n}, A ; Y\right)$ é equivalente a determinar o conível (Definição 1.23 de $Y^{*}$, que em princípio não é uma tarefa fácil. Assim, na próxima seção, calcularemos o conível de $\left(M^{k}\right)^{*}$, onde $M^{k}$ é uma variedade de dimensão $k$ que satisfaz algumas condições.

\subsection{PBU para $\left(\mathbb{S}^{n}, A ; M^{k}\right)$ e o conível de $\left(M^{k}\right)^{*}$}

Quando $X=\mathbb{S}^{n}$ e $Y=M^{k}$ é uma variedade de dimensão $k$, a validade da propriedade de Borsuk-Ulam para a terna $\left(\mathbb{S}^{n}, A ; M^{k}\right)$, foi bastante estudada, sem o auxílio da proposição anterior. Na verdade os resultados conhecidos juntamente com a proposição anterior permitem-nos dar um resultado geral sobre o conível de $\left(M^{k}\right)^{*}$. Quando $M^{k}$ é uma variedade diferenciável de dimensão $k$, Conner-Floyd provam o seguinte resultado:

Teorema 7.3. [6, Teorema 33.1] Sejam $f: \mathbb{S}^{n} \rightarrow M^{k}$ uma aplicação, e $A(f)=\{x \in$ $\left.\mathbb{S}^{n} \mid f(x)=f(-x)\right\}$. Então,

(i) se $n>k$, então $\operatorname{dim} A(f) \geq n-k$;

(ii) se $n=k$ e $f^{*}: H^{n}\left(M^{n} ; \mathbb{Z}_{2}\right) \rightarrow H^{n}\left(\mathbb{S}^{n} ; \mathbb{Z}_{2}\right)$ é trivial, então $A(f) \neq \emptyset$.

Este resultado foi generalizado para variedades topológicas por Munkolm em [29], e posteriormente para variedades generalizadas em [2]. Devido aos resultados acima e uma pequena observação podemos enunciar:

Proposição 7.4. Seja $M^{k}$ uma variedade topológica compacta. Para a terna $\left(\mathbb{S}^{n}, A ; M^{k}\right)$, a propriedade de Borsuk-Ulam

(i) vale para $n>k$;

(ii) vale para $n=k$, se toda aplicação $f: \mathbb{S}^{n} \rightarrow M^{n}$ é tal que $f^{*}: H^{n}\left(M^{n} ; \mathbb{Z}_{2}\right) \rightarrow$ $H^{n}\left(\mathbb{S}^{n} ; \mathbb{Z}_{2}\right)$ é trivial;

(iii) não vale para $n<k$. 
Demonstração. A validade de (i) segue diretamente da primeira parte do Teorema 7.3 . Pelo Teorema 7.3(ii), se toda aplicação $f: \mathbb{S}^{n} \rightarrow M^{n}$ tem a propriedade de que o homomorfismo induzido em cohomologia no nível $n$ (com coeficientes em $\mathbb{Z}_{2}$ ) é trivial, então a propriedade de Borsuk-Ulam vale para $\left(\mathbb{S}^{n}, A ; M^{n}\right)$, e isto mostra (ii). Para provarmos que a propriedade de Borsuk-Ulam não vale para $\left(\mathbb{S}^{n}, A ; M^{k}\right)$ para $n<k$, é suficiente construirmos uma aplicação equivariante em $\left(M^{k}\right)^{*}$. Para isto, seja $\phi: \mathbb{S}^{n} \rightarrow M^{k}$ um mergulho e consideremos $f: \mathbb{S}^{n} \rightarrow\left(M^{k}\right)^{*}$ dada por $f(x)=(\phi(x), \phi(-x))$. A aplicação $f$ é equivariante, e daí (iii) segue da Proposição 7.1.

Assim, quanto ao conível de $\left(M^{k}\right)^{*}$, temos:

Proposição 7.5. Seja $M^{k}$ uma variedade topológica compacta. Então, $s^{\prime}\left(\left(M^{k}\right)^{*}\right)=k$ ou $k+1$.

Demonstração. Das proposições 7.4 e 7.1, não existe aplicação equivariante de $\mathbb{S}^{k+1} \mathrm{em}$ $\left(M^{k}\right)^{*}$, pois a propriedade de Borsuk-Ulam vale para $\left(\mathbb{S}^{k+1}, A ; M^{k}\right)$, e existe aplicação equivariante de $\mathbb{S}^{k-1}$ em $\left(M^{k}\right)^{*}$, pois a propriedade de Borsuk-Ulam não vale para $\left(\mathbb{S}^{k-1}, A ; M^{k}\right)$. Assim, pela Definição 1.23 , segue que $k \leq s^{\prime}\left(\left(M^{k}\right)^{*}\right) \leq k+1$.

Dada uma aplicação $f: \mathbb{S}^{n} \rightarrow M^{n}$, pelos resultados anteriores se $f^{*}: H^{n}\left(M^{n} ; \mathbb{Z}_{2}\right) \rightarrow$ $H^{n}\left(\mathbb{S}^{n} ; \mathbb{Z}_{2}\right)$ é trivial, então a aplicação $f$, assim como qualquer outra homotópica a ela, tem uma coincidência antipodal. Se $f^{*}: H^{n}\left(M^{n} ; \mathbb{Z}_{2}\right) \rightarrow H^{n}\left(\mathbb{S}^{n} ; \mathbb{Z}_{2}\right)$ é não trivial, não sabemos se existe uma $g$ homotópica a $f$ tal que $g$ não tenha coincidência antipodal. Se isto for verdade implicaria que a propriedade de Borsuk-Ulam não valeria, e portanto a segunda condição do teorema anterior seria necessária e suficiente.

Seja $M^{k}$ uma variedade topológica que tem a cohomologia da esfera, com coeficientes $\mathbb{Z}_{2}$, isto é, $H^{i}\left(M^{k} ; \mathbb{Z}_{2}\right)=0$ para todo $0<i<k$, a qual chamamos de uma esfera de cohomologia módulo 2. Logo, da condição dada pelo item (ii) da proposição anterior, para variedades topológicas que não são esfera de cohomologia módulo 2, mostramos:

Proposição 7.6. Seja $M^{k}$ uma variedade topológica compacta que não é uma esfera de cohomologia módulo 2. Então, a terna $\left(\mathbb{S}^{n}, A ; M^{k}\right)$ tem a propriedade de Borsuk-Ulam para todo $n \geq k$.

Demonstração. Já sabemos de sua validade para $n>k$. Agora, para $n=k$, consideremos uma aplicação $f: \mathbb{S}^{k} \rightarrow M^{k}$. Como $M^{k}$ não é uma esfera de cohomologia módulo 2 , existe 
$0<j<k$ tal que $H^{j}\left(M^{k} ; \mathbb{Z}_{2}\right) \neq 0$. Assim, seja $0 \neq \alpha \in H^{j}\left(M^{k} ; \mathbb{Z}_{2}\right)$. Da Dualidade de Poincaré, [8, Proposição 8.13], existe $\beta \in H^{k-j}\left(M^{k} ; \mathbb{Z}_{2}\right)$ de modo que

$$
\alpha \smile \beta=u,
$$

onde $u \in H^{k}\left(M^{k} ; \mathbb{Z}_{2}\right)$ é a classe fundamental. Logo,

$$
f^{*}(\alpha \smile \beta)=f^{*}(u) \Rightarrow f^{*}(\alpha) \smile f^{*}(\beta)=f^{*}(u) \Rightarrow f^{*}(u)=0 \smile f^{*}(\beta)=0 .
$$

Portanto, pela Proposição $7.4\left(\right.$ ii), a propriedade de Borsuk-Ulam vale para $\left(\mathbb{S}^{k}, A ; M^{k}\right)$.

Proposição 7.7. Nas hipóteses da proposição anterior, $s^{\prime}\left(\left(M^{k}\right)^{*}\right)=k$.

Demonstração. Das Proposições 7.6 e 7.1, não existe aplicação equivariante de $\mathbb{S}^{k}$ em $\left(M^{k}\right)^{*}$. Portanto, o resultado segue da Proposição 7.5 . 


\section{Apêndice A}

\section{Involuções livres sobre $K b \times K b$}

Neste apêndice, apresentaremos alguns cálculos na tentativa de encontrarmos outras involuções livres sobre o produto $K b \times K b$, que não sejam da forma diagonal. Para isto, consideraremos a 4 -variedade quociente $W:=(K b \times K b) / \tau_{1}$, onde $\tau_{1}$ é como no Capítulo 6. para assim determinarmos uma apresentação do grupo fundamental de cada um de seus revestimentos duplos e verificarmos quais deles correspondem a $K b \times K b$, cujo procedimento já foi utilizado no Capítulo 5. Como veremos, não será possível encontrar tais involuções livres sobre $K b \times K b$, neste estudo em particular. No Lema 6.1, vimos que $W$ tem o mesmo tipo de homotopia do espaço total de um $T^{2}$-fibrado sobre $T^{2}$, e consequentemente seus revestimentos duplos também (prova do Teorema 3.5). Assim, aproveitaremos os cálculos aqui realizados, para observar que alguns destes revestimentos também têm o mesmo tipo de homotopia do espaço total de um $T^{2}$-fibrado sobre $K b$, ou de um $K b$-fibrado sobre $T^{2}$.

\section{A.1 Cálculos}

No Capítulo 6, vimos que

$$
\pi_{1}(W)=\left\langle x, y, z, w \mid[x, y]^{\prime},[x, z],[x, w],[y, w],[z, y] x,[z, w]^{\prime} x\right\rangle
$$

e que são sete os revestimentos duplos de $W$, que denotamos por $W_{i}$, associados aos epimorfismos $\varphi_{i}: \pi_{1}(W) \rightarrow \mathbb{Z}_{2}, i=1, \ldots, 7$, conforme a Tabela 6.1. Na sequência, determinaremos uma apresentação para $\pi_{1}\left(W_{i}\right)=\operatorname{Ker}\left(\varphi_{i}\right)$, para cada $i=1, \ldots, 7$, e analisaremos para quais $i$ 's temos $W_{i} \simeq K b \times K b$.

(1) Seja $\{1, y\}$ um sistema de Schreier. Pelo método de Reidemeister-Schreier, $\pi_{1}\left(W_{1}\right)=$ 
$\operatorname{Ker}\left(\varphi_{1}\right)$ tem os seguintes geradores:

$$
\begin{aligned}
& y_{11}=\varrho(1, x)=x \cdot(\bar{x})^{-1}=x, \\
& y_{12}=\varrho(y, x)=y x \cdot(\overline{y x})^{-1}=y x y^{-1}, \\
& y_{21}=\varrho(1, y)=y \cdot(\bar{y})^{-1}=1, \\
& y_{22}=\varrho(y, y)=y^{2} \cdot\left(\overline{y^{2}}\right)^{-1}=y^{2}, \\
& y_{31}=\varrho(1, z)=z \cdot(\bar{z})^{-1}=z, \\
& y_{32}=\varrho(y, z)=y z \cdot(\overline{y z})^{-1}=y z y^{-1}, \\
& y_{41}=\varrho(1, w)=w \cdot(\bar{w})^{-1}=w, \\
& y_{42}=\varrho(y, w)=y w \cdot(\overline{y w})^{-1}=y w y^{-1}, \\
& \text { e as seguintes relações: }
\end{aligned}
$$$$
y_{11} y_{12}=1 \text {, }
$$

$y_{12} y_{22} y_{11} y_{22}^{-1}=1$,

$\left[y_{11}, y_{31}\right]=1$,

$\left[y_{12}, y_{32}\right]=1$,

$\left[y_{11}, y_{41}\right]=1$,

$\left[y_{12}, y_{42}\right]=1$,

$y_{42} y_{41}^{-1}=1$,

$y_{22} y_{41} y_{22}^{-1} y_{42}^{-1}=1$,

$y_{31} y_{32}^{-1} y_{12}^{-1}=1$,

$y_{32} y_{22} y_{31}^{-1} y_{11}^{-1} y_{22}^{-1}=1$,

$\left[y_{31}, y_{41}\right]^{\prime} y_{11}=1$

$\left[y_{32}, y_{42}\right]^{\prime} y_{12}=1$

De A.1 e A.7), segue que $y_{12}=y_{11}^{-1}$ e $y_{42}=y_{41}$, respectivamente. Assim, de A.2 e A.8), temos $\left[y_{11}, y_{22}\right]=1$ e $\left[y_{22}, y_{41}\right]=1$, respectivamente. De (A.9), $y_{32}=y_{11} y_{31}$, e consequentemente de A.10 vem que $\left[y_{22}, y_{31}\right]=1$. Com estas igualdades, as relações A.4, A.6 e A.12, são iguais a A.3), A.5 e A.11, respectivamente. Daí, com a notação: $z_{1}=y_{11}, z_{2}=y_{22}, z_{3}=y_{31}$ e $z_{4}=y_{41}$, temos

$$
\pi_{1}\left(W_{1}\right)=\left\langle z_{1}, z_{2}, z_{3}, z_{4} \mid\left[z_{1}, z_{2}\right],\left[z_{1}, z_{3}\right],\left[z_{1}, z_{4}\right],\left[z_{2}, z_{4}\right],\left[z_{2}, z_{3}\right],\left[z_{3}, z_{4}\right]^{\prime} z_{1}\right\rangle .
$$


Observemos que $\pi_{1}(K b \times K b)_{a b} \cong \mathbb{Z}^{2} \oplus \mathbb{Z}_{2} \oplus \mathbb{Z}_{2}$, enquanto que

$$
\begin{aligned}
\pi_{1}\left(W_{1}\right)_{a b} & =\left\langle z_{1}, z_{2}, z_{3}, z_{4} \mid\left[z_{1}, z_{2}\right],\left[z_{1}, z_{3}\right],\left[z_{1}, z_{4}\right],\left[z_{2}, z_{3}\right],\left[z_{2}, z_{4}\right],\left[z_{3}, z_{4}\right], z_{1}=z_{3}^{-2}\right\rangle \\
& =\left\langle z_{2}, z_{3}, z_{4} \mid\left[z_{2}, z_{3}\right],\left[z_{2}, z_{4}\right],\left[z_{3}, z_{4}\right]\right\rangle \\
& \cong \mathbb{Z}^{3}
\end{aligned}
$$

Portanto, $W_{1} \nsucceq K b \times K b$.

(2) Da prova do Teorema 2.8, para o caso particular em que $M_{1}=M_{2}=K b$, o epimorfismo $\varphi: \pi_{1}(W) \rightarrow \mathbb{Z}_{2}$ associado ao revestimento duplo $K b \times K b \rightarrow W$ é tal que $\varphi(x)=\kappa^{-1}(\psi, \phi)\left(u^{2}\right)=\kappa^{-1}(\overline{0}, \overline{0})=\overline{0}$, $\varphi(y)=\kappa^{-1}(\psi, \phi)(v)=\kappa^{-1}(\overline{0}, \overline{0})=\overline{0}$, $\varphi(z)=\kappa^{-1}(\psi, \phi)\left(\widetilde{u} u^{-1}\right)=\kappa^{-1}(\overline{1}, \overline{1})=\overline{1}$, $\varphi(w)=\kappa^{-1}(\psi, \phi)(\widetilde{v})=\kappa^{-1}(\overline{0}, \overline{0})=\overline{0}$, ou seja, $\varphi=\varphi_{2}$, e portanto $W_{2}=K b \times K b$.

Assim como em (1), para os demais revestimentos temos:

(3) $\pi_{1}\left(W_{3}\right)=\left\langle z_{1}, z_{2}, z_{3}, z_{4} \mid\left[z_{1}, z_{2}\right]^{\prime},\left[z_{1}, z_{3}\right],\left[z_{1}, z_{4}\right],\left[z_{2}, z_{4}\right],\left[z_{3}, z_{4}\right],\left[z_{3}, z_{2}\right] z_{1}\right\rangle$, onde $z_{1}=x$, $z_{2}=y, z_{3}=z$ e $z_{4}=w^{2}$.

Como $\pi_{1}\left(W_{3}\right)_{a b} \cong \mathbb{Z}^{3}$, segue que $W_{3} \not K b \times K b$.

(4) $\pi_{1}\left(W_{4}\right)=\left\langle z_{1}, z_{2}, z_{3}, z_{4} \mid\left[z_{1}, z_{2}\right],\left[z_{1}, z_{3}\right]^{\prime},\left[z_{1}, z_{4}\right],\left[z_{2}, z_{4}\right],\left[z_{2}, z_{3}\right],\left[z_{3}, z_{4}\right]^{\prime} z_{2}\right\rangle$, onde $z_{1}=x$, $z_{2}=y^{2}, z_{3}=z y^{-1}$ e $z_{4}=w$.

Como $\pi_{1}\left(W_{4}\right)_{a b} \cong \mathbb{Z}^{2} \oplus \mathbb{Z}_{2}$, segue que $W_{4} \not K b \times K b$.

(5) $\pi_{1}\left(W_{5}\right)=\left\langle z_{1}, z_{2}, z_{3}, z_{4} \mid\left[z_{1}, z_{2}\right],\left[z_{1}, z_{3}\right],\left[z_{1}, z_{4}\right]^{\prime},\left[z_{2}, z_{4}\right],\left[z_{2}, z_{3}\right],\left[z_{3}, z_{4}\right]^{\prime}\right\rangle$, onde $z_{1}=x$, $z_{2}=y^{2}, z_{3}=z$ e $z_{4}=w y^{-1}$.

Suponhamos que

$$
\pi_{1}\left(W_{5}\right) \stackrel{\psi}{\cong} \pi_{1}(K b \times K b)=\left\langle a_{1}, a_{2}, b_{1}, b_{2} \mid\left[a_{1}, a_{2}\right]^{\prime},\left[b_{1}, b_{2}\right]^{\prime},\left[a_{i}, b_{j}\right], 1 \leq i, j \leq 2\right\rangle .
$$

Daí, como $z_{2} \in Z\left(\pi_{1}\left(W_{5}\right)\right)$, temos $\psi\left(z_{2}\right) \in Z\left(\pi_{1}(K b \times K b)\right)=\left\langle a_{2}^{2}, b_{2}^{2}\right\rangle$. Logo, se $\psi\left(z_{2}\right)=a_{2}^{2}$, então

$$
z_{2}=\psi^{-1}\left(a_{2}^{2}\right)=\left(\psi^{-1}\left(a_{2}\right)\right)^{2}
$$

o que é uma contradição. O mesmo acontece se $\psi\left(z_{2}\right)=b_{2}^{2}$. Portanto, concluímos que $W_{5} \not 千 K b \times K b$.

(6) $\pi_{1}\left(W_{6}\right)=\left\langle z_{1}, z_{2}, z_{3}, z_{4} \mid\left[z_{1}, z_{2}\right]^{\prime},\left[z_{1}, z_{3}\right],\left[z_{1}, z_{4}\right],\left[z_{2}, z_{4}\right] z_{1},\left[z_{3}, z_{2}\right] z_{1}^{2},\left[z_{3}, z_{4}\right]^{\prime} z_{1}^{2}\right\rangle$, onde $z_{1}=$ $x, z_{2}=y, z_{3}=z^{2}$ e $z_{4}=w z^{-1}$.

Portanto, $W_{6} \nsucceq K b \times K b$ pois $\pi_{1}\left(W_{6}\right)_{a b} \cong \mathbb{Z}^{2} \oplus \mathbb{Z}_{2}$. 
(7) $\pi_{1}\left(W_{7}\right)=\left\langle z_{1}, z_{2}, z_{3}, z_{4} \mid\left[z_{1}, z_{2}\right],\left[z_{1}, z_{3}\right]^{\prime},\left[z_{1}, z_{4}\right]^{\prime},\left[z_{2}, z_{4}\right], z_{3}^{2} z_{4}^{2},\left[z_{2}, z_{3}\right]\right\rangle$, onde $z_{1}=x, z_{2}=$ $y^{2}, z_{3}=z y^{-1}$ e $z_{4}=w y^{-1}$.

Uma vez que $z_{2} \in Z\left(\pi_{1}\left(W_{7}\right)\right)$, de modo análogo a (5), $W_{7} \not K b \times K b$.

Conclusão. Apenas um dos sete revestimentos duplos de $(K b \times K b) / \tau_{1}$ corresponde a $K b \times K b$. Assim, neste estudo em particular, não foi possível detectarmos uma involução livre sobre $K b \times K b$, que não seja da forma diagonal.

Observação A.1. Sabemos que $W$ e $W_{i}$ têm o mesmo tipo de homotopia do espaço total de um $T^{2}$-fibrado sobre $T^{2}$ (Capítulo 6), com $i=1, \ldots, 7$. Mostra-se também que $W_{5}$ e $W_{7}$ têm o mesmo tipo de homotopia do espaço total de um $T^{2}$-fibrado sobre $K b$, e que $W_{3}$ tem o mesmo tipo de homotopia do espaço total de um $K b$-fibrado sobre $T^{2}$. 


\section{Referências Bibliográficas}

[1] Bauval, A., Gonçalves, D. L., Hayat, C., And Zvengrowski, P. The Borsuk-Ulam theorem for the manifolds of geometry spherical, $\mathbb{S}^{2} \times \mathbb{R}$, flat, and Nil: in preparation.

[2] Biasi, C., de Mattos, D., And dos Santos, E. L. A Borsuk-Ulam theorem for maps from a sphere to a generalized manifold. Geometriae Dedicata, 107 (2004), 101-110.

[3] Borsuk, K. Drei Sätze über die n-dimensionale euklidische Sphäre. Fund. Math., 20 (1933), 177-190.

[4] Bredon, G. E. Introduction to compact transformation groups, vol. 46 of Pure and Applied Mathematics. Academic Press, New York and London, 1992.

[5] Brown, K. S. Cohomology of Groups, vol. 87 of Graduate Texts in Mathematics. Springer Verlag, New York-Berlin, 1982.

[6] Conner, P. E., And Floyd, E. E. Differentiable Periodic Maps. Springer Verlag, Berlin-Göttingen-Heidelberg, 1964.

[7] Dai, Z. D., AND LAM, T. Y. Levels in algebra and topology. Comment. Math. Helvetici, 59 (1984), 376-424.

[8] Dold, A. Lectures on Algebraic Topology. Springer-Verlag, Berlin-Heidelberg-New York, 1972.

[9] Earle, C. J., And Eells, J. A fibre bundle description of Teichmüller theory. J. Differential Geometry 3 (1969), 19-43.

[10] Eckmann, B., And Müller, H. Poincaré duality groups of dimension two. Comment. Math. Helvetici, 55 (1980), 510-520. 
[11] Gonçalves, D. L. The Borsuk-Ulam theorem for surfaces. Quaestiones Mathematicae, 29 (2006), 117-123.

[12] Gonçalves, D. L., And Guaschi, J. The Borsuk-Ulam theorem for maps into a surface. Topology and its Applications, 157 (2010), 1742-1759.

[13] Gonçalves, D. L., Hayat, C., And Zvengrowski, P. The Borsuk-Ulam theorem for manifolds, with applications to dimensions two and three. Proceedings Bratislava Topology Symposium. Group Actions and Homogeneous Spaces (2010), 9-28.

[14] Gonçalves, D. L., Neto, O. M., And Spreafico, M. The Borsuk-Ulam theorem for homotopy spherical space forms. Journal of Fixed Point Theory and Applications 9, 2 (2011), 285-294.

[15] Gonçalves, D. L., Penteado, D., and Vieira, J. P. Fixed points on torus fiber bundles over the circle. Fundamenta Mathematicae 183, 1 (2004), 1-38.

[16] Gottlieb, D. H. A certain subgroup of the fundamental group. American Journal of Mathematics 87, 4 (1965), 840-856.

[17] Gottlieb, D. H. On fibre spaces and evaluation map. Annals of Mathematics 87, 1 (1968), 42-55.

[18] Hillman, J. A. Flat 4-manifold groups. New Zealand Journal of Mathematics 24 (1995), 29-40.

[19] Hillman, J. A. Four-manifolds, geometries and knots, vol. 5 of Geometry and Topology Monographs. 2002.

[20] Husemoller, D. Fibre Bundles, vol. 20 of Graduate Texts in Mathematics. Springer Verlag, 1994.

[21] Johnson, D. L. Presentations of Groups, vol. 15 of London Mathematical Society, Student Texts. Cambridge University Press, 1997.

[22] Kozlov, D. Combinatorial Algebraic Topology, vol. 21 of Algorithms and Computation in Mathematics. Springer-Verlag, Berlin Heidelberg, 2008. 
[23] Magnus, W., Karrass, A., And Solitar, D. Combinatorial Group Theory: presentations of groups in terms of generators and relations, vol. XIII of Pure and Applied Mathematics: A series of texts and monographs. Interscience Publishers, 1966.

[24] Massey, W. S. Algebraic Topology: An Introduction, vol. 56 of Graduate Texts in Mathematics. Springer Verlag, 1967.

[25] MatoušEK, J. Using the Borsuk-Ulam Theorem. Lectures on Topological Methods in Combinatorics and Geometry. Springer-Verlag, Berlin-Heidelberg-New York, 2003.

[26] Melvin, P. 2-sphere bundles over compact surfaces. Proceedings of the Am. Math. Soc. 92, 4 (1984), 567-572.

[27] Milgram, R. J. The bar construction and abelian H-spaces. Illinois J. Math. 11 (1967), 242-250.

[28] Milnor, J. W., And Stasheff, J. D. Characteristic Classes. No. 76 in Annals of Mathematics Studies. Princeton University Press and University of Tokyo Press, Princeton, New Jersey, 1974.

[29] Munkholm, H. J. A Borsuk-Ulam theorem for maps from a sphere to compact topological manifold. Illinois J. Math., 13 (1969), 116-124.

[30] Murasugi, K., And Kurpita, B. I. A study of braids, vol. 484 of Mathematics and its Applications. Kluwer Academic Publishers, Dordrecht, 1999.

[31] Robinson, D. J. S. A Course in the Theory of Groups, vol. 80 Second Edition of Graduate Texts in Mathematics. Springer Verlag, 1996.

[32] Spanier, E. H. Algebraic Topology. McGraw-Hill Book Company, 1966.

[33] Steenrod, N. The Topology of Fibre Bundles. Princeton landmarks in mathematics and physics. Princeton University Press, Princeton, New Jersey, 1999.

[34] Vendrúscolo, D., Desideri, P. E., And Pergher, P. L. Q. Some generalizations of the Borsuk-Ulam theorem. Publicationes Mathematicae (Debrecen), 78 (2011), 583-593. 
[35] Whitehead, G. W. Elements of Homotopy Theory, vol. 61 of Graduate texts in Mathematics. Springer-Verlag, New York, 1978. 


\section{Índice Remissivo}

Aplicação

equivariante, 3

classificante, 2

Borsuk-Ulam

Propriedade de, 4

Teorema de, 4

Conível, 6

Diagonal, 76

Esfera

de cohomologia módulo 2, 78

Espaço

classificante, 2

Fibrado

de superfície, 21

Grupo de

n-variedade flat, 59

superfície, 56

Homomorfismo

conectante, 21

Involução, 3

antipodal, 3

diagonal, 9

livre, 3
Nível, 6

Sequência

de homotopia da fibração, 3

Superfície

fechada, 1 